Pacific Northwest

National Laboratory

Operated by Battelle for the

U.S. Department of Energy

\section{Vitrification and Product Testing of C-104 and AZ-102 \\ Pretreated Sludge Mixed with Flowsheet Quantities of Secondary Wastes}

February 2001

M.J. Schweiger

H.D. Smith

M.W. Urie

J.J. Wagner

L.R. Greenwood

R.C. Lettau

G.F. Piepel

Prepared for CH2M Hill Hanford Group, Inc. under Project Number 41503 


\section{DISCLAIMER}

This report was prepared as an account of work sponsored by an agency of the United States Government. Neither the United States Government nor any agency thereof, nor Battelle Memorial Institute, nor any of their employees, makes any warranty, express or implied, or assumes any legal liability or responsibility for the accuracy, completeness, or usefulness of any information, apparatus, product, or process disclosed, or represents that its use would not infringe privately owned rights. Reference herein to any specific commercial product, process, or service by trade name, trademark, manufacturer, or otherwise does not necessarily constitute or imply its endorsement, recommendation, or favoring by the United States Government or any agency thereof, or Battelle Memorial Institute. The views and opinions of authors expressed herein do not necessarily state or reflect those of the United States Government or any agency thereof.

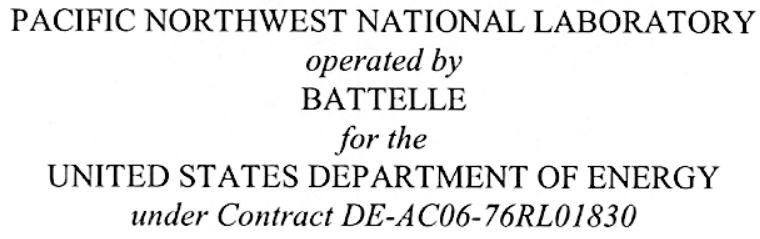

Printed in the United States of America

$$
\begin{aligned}
& \text { Available to DOE and DOE contractors from the } \\
& \text { Office of Scientific and Technical Information, } \\
& \text { P.O. Box 62, Oak Ridge, TN 37831-0062; } \\
& \text { ph: (865) 576-8401 } \\
& \text { fax: (865) 576-5728 } \\
& \text { email: reports@adonis.osti.gov }
\end{aligned}
$$

Available to the public from the National Technical Information Service, U.S. Department of Commerce, 5285 Port Royal Rd., Springfield, VA 2216 ph: (800) 553-6847 fax: (703) 605-6900

email: orders@ntis.fedworld.gov online ordering: http://www.ntis.gov/ordering.htm 
PNNL-13452

WTP-RPT-006, Rev. 0

(Formerly BNFL-RPT-042)

\title{
Vitrification and Product Testing of C-104 and AZ-102 Pretreated Sludge Mixed with Flowsheet Quantities of Secondary Wastes
}

\author{
G. L. Smith \\ D. R. Bates \\ R. W. Goles \\ L. R. Greenwood \\ R. C. Lettau \\ G. F. Piepel \\ M. J. Schweiger \\ H. D. Smith \\ M. W. Urie \\ J. J. Wagner
}

February 2001

Prepared for

CH2M Hill Hanford Group, Inc.

under Project Number 41503

Pacific Northwest National Laboratory

Richland, Washington 99352 


\section{Summary}

The U.S. Department of Energy (DOE) Office of River Protection (ORP) has acquired Hanford tank waste treatment services at a demonstration scale. The River Protection Project Waste Treatment Plant (RPP-WTP) team is responsible for producing an immobilized (vitrified) high-level waste (IHLW) waste form. Pacific Northwest National Laboratory, hereafter referred to as PNNL, has been contracted to produce and test a vitrified IHLW waste form from two Envelope D high-level waste (HLW) samples previously supplied to the RPP-WTP project by DOE.

The primary objective for vitrifying the HLW samples is to generate glass products for subsequent product testing. The scope of the Vitrification and Product Testing has been divided into eight work elements: 1) Glass Fabrication, 2) Chemical Composition, 3) Radiochemical Composition, 4) Crystalline and Non-crystalline Phase Determination, 5) Release Rate (PCT), 6) Toxicity Characteristic Leaching Procedure (TCLP), 7) Total volatile organic and semi-volatile organic analyses (VOA and SVOA), and 8) WAPS, regulatory, and de-listing testing. The work presented in this report is from only the following 5 work elements: 1) Glass Fabrication, 2) Chemical Composition, 3) Radiochemical Composition, 4) Crystalline and Non-crystalline Phase Determination, and 5) Release Rate (PCT). These work elements will help demonstrate the RPP-WTP projects ability to satisfy the product requirements concerning, chemical and radionuclide reporting, waste loading, identification and quantification of crystalline and non-crystalline phases, and waste form leachability. Results from work elements 6 through 8, i.e. VOA, SVOA, dioxins, furans, PCBs, and total cyanide and sulfide analyses are reported in a separate document (Goheen et al., WTP-RPT-010).

Two pretreated tank sludge samples, high-level wastes (241-C-104 and 241-AZ-102) hereafter referred to as C-104 and AZ-102 along with a HLW process simulant (know as the HLW Process Blank) were prepared as melter feeds for vitrification. Due to scheduling constraints and small initial sample size of the pretreated tank 241-AZ-102 sludge, this sample was divided into two samples that were vitrified separately (i.e. AZ-102, Melt 1 and AZ-102, Melt 2). The analyzed compositions of the pretreated C-104 and AZ-102 sludge wastes were used by Catholic University of America's (CUA) Vitreous State Laboratory (VSL) to determine the target glass composition.

The two tank sludge samples, were processed through pretreatment chemical washing and leaching processes, and the pretreated sludges were converted to high-level waste (HLW) glass after flowsheet quantities of secondary wastes, i.e. Sr/TRU precipitate and Cs and Tc ion exchange eluants, generated from LAW supernatant pretreatment unit operations were added. Both sludge samples were processed through the following unit operations to simulate the RPP-WTP project flowsheet: 1) initial characterization; 2) washing; 3) leaching; and 4) filtration in a crossflow filtration system (Brooks et al., 2000a) (Brooks et al., 2000b). Additional washing/leaching and solubility versus temperature studies were completed on a small subsample of C-104 tank sludge that was not vitrified (Lumetta et al., 2000). In addition to the initial characterization of the C-104 tank sludge, subsamples were extensively analyzed for inorganic, radiochemical, and organic constituents (Evans et al., 2000 and Fiskum et al., 2000). Physical and rheological testing of the C-104 and AZ-102 pretreated sludge and melter feeds has also been completed and reported in a separate document (Bredt et al., 2000).

Pretreated tank wastes were vigorously blended in a stainless steel beaker using a magnetic stirrer and stir bar. Secondary wastes (Sr/TRU precipitate, the composite Cs ion exchange eluant, and Tc ion exchange eluants) were combined with the pretreated tank sludge waste into the same stainless steel beaker. A combination of glass former additives Borax $\left(\mathrm{Na}_{2} \mathrm{~B}_{4} \mathrm{O}_{7} \cdot 10 \mathrm{H}_{2} \mathrm{O}\right)$; Lithium Hydroxide Monohydrate $\left(\mathrm{LiOH} \cdot \mathrm{H}_{2} \mathrm{O}\right)$ Silica sand $\left(\mathrm{SiO}_{2}\right)$; Zinc Oxide $(\mathrm{ZnO})$; and sugar) were added to each 
pretreated waste to produce a melter feed. The C-104 and AZ-102 melter feeds were dried, calcined, and melted at $1150^{\circ} \mathrm{C}$ for two hours. The glass melt was then poured onto a stainless steel plate (air quenched), cooled to room temperature, and handled in a manner to keep the glass free of organic contamination. All glass samples were stored in glassware cleaned to EPA standards. A portion of the melt was poured into a small box crucible that was later heat-treated following the predicted canister centerline cooling (CCC) heat treatment of a Hanford HLW canister. The final C-104 melt pour was non-problematic with an estimated viscosity of about $15 \mathrm{~Pa} \cdot \mathrm{s}$ based on visual observation coupled with past experience. The final AZ-102 Melt 1 pour temperature was elevated to approximately $1200^{\circ} \mathrm{C}$ due to the smaller sample size; this pour was non-problematic with an estimated viscosity of about $5 \mathrm{~Pa} \cdot \mathrm{s}$, based on visual observation. The final AZ-102 Melt 2 pour temperature was also elevated to approximately $1200^{\circ} \mathrm{C}$ due to the smaller sample size; the pour was non-problematic as well, with an estimated viscosity of about $5 \mathrm{~Pa} \cdot \mathrm{s}$, based on visual observation coupled with past experience.

Vitrification of slurry melter feed in an actual melter progresses continuously through 3 distinct stages, drying, calcining and melting. Under steady-state operating conditions, the aqueous slurry that is introduced into the high-temperature melter environment spreads out over an existing cold cap where it dries and becomes part of the melter cold-cap structure. This dried material begins working its way down through the cold-cap as it becomes submerged in incoming feed while, at the same time, material, at the molten-glass/cold-cap interface, is dissolving into the glass melt. During this continuous progression through the cold cap, the temperature that the feed is subjected to monotonically increases from the boiling point of water $\left(\sim 100^{\circ} \mathrm{C}\right)$ to molten glass temperatures $\left(\sim 1150{ }^{\circ} \mathrm{C}\right)$. Accompanying this continuous physical and thermal transition, inorganic eutectic salts are slowly converted to their oxide forms (calcined) that are suitable for subsequent incorporation into the melter's molten glass pool.

All of these discrete phases of liquid-fed ceramic melter (LFCM) feed processing have been faithfully reproduced in the crucible studies performed. What may not be truly represented, however, is the complex stages and nature of the cold-cap chemistry that results in the calcination of the feed material. For non-volatile, inorganic feed constituent, the differences between crucible and melter vitrification conditions are inconsequential. For all other feed components, cold-cap chemistry can influence both partitioning behavior and chemical byproduct yields, which, in turn, can and will affect the resultant glass product.

Consequently, to properly represent an LFCM glass product, actual physical and chemical processing conditions need to be replicated. But since this requires the development of a representative cold-cap structure, nothing short of a liquid-fed melting process (e.g., scaled melter or possibly a gradient furnace test) is truly adequate. However, relationships drawn between previous crucible and actual melter testing results, i.e. from VSL and GTS Duratek testing, that were conducted using a fixed feed may be useful in extracting reference glass-product quality parameters from extrapolated crucible test data.

One of the objectives for the glass product waste loading for the HLW glasses was to meet the requirements delineated in the RPP-WTP Phase B-1 contract, specification 1.2.2.1.6, titled Product Loading, which states: "Loading of non-volatile components in Envelope D, and, if directed by DOE, entrained solids after washing in accordance with Specification 12, Number of HLW Canister Per Batch of Waste Envelope D, shall be achieved, such that, the concentration of at least one of the waste components or waste component combinations in Table TS-1.1 Minimum Component Limits in HLW Glass exceeds its minimum weight percent in HLW glass as identified in Table TS-1.1 ..." The total of all waste oxides (exclusive of Si) not identified in Table TS-1.1 was calculated for the C-104 glass, taking into account the glass former minerals added, using the "Average Normalized" weight percent oxide values. The total waste percent total oxide in the C-104 glass, per the above conditions, comes to $10.02 \%$, which is greater than the required $8.0 \%$. The total of $\mathrm{Al}_{2} \mathrm{O}_{3}+\mathrm{Fe}_{2} \mathrm{O}_{3}+\mathrm{ZrO}_{2}$ for AZ-102 Melt 1 
is $21.96 \%$ and for AZ-102 Melt 2 is $21.61 \%$ which is greater than the required $21.0 \%$. Therefore, the C-104, AZ-102 Melt 1, and AZ-102 Melt 2 glasses easily meet the ORP contract specifications for waste product loading. The total accountability of mass in these glasses by ICP-AES ranges from 92.4 to $101.8 \%$ for the Envelope D glasses AZ-102 Melt 1, AZ-102 Melt 2 and C-104. The discrepancy in total wt $\%$ oxides is because certain elements (such as $\mathrm{SO}_{3}$; the halides $\mathrm{Br}, \mathrm{Cl}$, and $\mathrm{F}$; and trace metals) were not included in the analyses. Another reason is the possible incomplete recovery of $\mathrm{SiO}_{2}$ during the preparation of the sample for analysis and the difficulty in optimizing $\mathrm{Na}_{2} \mathrm{O}$ detection. Adjustments have been made to the measured data to generate as realistic as possible estimates of the composition of each of the three glasses due to potential analytical problems caused by: analytical detection limits greater than target values and possible analytical bias. The approach taken for undetected elements/oxides was to use their target values as the measurement instead of a blank measurement. The approach taken for bias correction was to analyze a well characterized glass, i.e. Analytical Reference Glass-1 (ARG-1), at the same time as these three glasses to evaluate potential biases between measured $\mathrm{wt} \%$ oxides in a glass sample and the true wt $\%$ oxides in the glass. The normalized adjusted composition total wt\% values for the adjusted AZ-102 Melt 1, AZ-102 Melt 2, and C-104 compositions are close enough to $100 \mathrm{wt} \%$ to renormalize the adjusted compositions so they total $100 \mathrm{wt} \%$; these normalized compositions are reported in this report.

The waste loading was calculated from the dilution factor (decrease in concentration) of elements contained in either the waste or the glass forming additives. The results indicate that the waste fraction of each glass is near their target, i.e. $33.1 \%$ for C-104 (measured 33.59\% based on waste dilution and 31.05\% based on additive dilution), 33.39\% for AZ-102 Melt 1 (measured 33.14\% based on waste dilution and 34.16\% based on additive dilution), and 33.97\% for AZ-102 Melt 2 (measured 33.98\% based on waste dilution and $34.22 \%$ based on additive dilution). The measured glass to target composition percent difference comparison of the oxides is small and the calculated waste loading values are very close to or exceed the target. Both support the conclusion that the actual waste loading in each glass met or exceeded the target waste loading.

To demonstrate that the IHLW glass product, radionuclide compositional contract criteria were met, it was assumed that each HLW glass, i.e. C-104, AZ-102 Melt 1, and AZ-102 Melt 2, are separate "waste types" and as such would fill multiple Hanford HLW canisters and that the HLW canister can be modeled as a right circular cylinder of $4.5 \mathrm{~m}$ height and $0.61 \mathrm{~m}$ diameter with a $100 \%$ glass fill of approximately $1.27 \mathrm{~m}^{3}$. The primary success objectives accomplished with this work are: 1) "the inventory of radionuclides (in Curies) that have half-lives longer than 10 years and that are, or will be, present in concentrations greater than 0.05 percent of the total radioactive inventory for each waste type, indexed to the years 2015 and 3115" are reported; 2) the total and fissile uranium and plutonium (U-233, $\mathrm{U}-235, \mathrm{Pu}-239$, and $\mathrm{Pu}-241)$ content of each canister of waste glass were calculated and are: 1161.5 grams for C-104, 642.1 grams for AZ-102 Melt 1, and 619.4 grams for AZ-102 Melt 2; 3) the concentration of plutonium in grams per cubic meter of each waste glass are: $124.7 \mathrm{~g} / \mathrm{m}^{3}$ for C-104, $122.4 \mathrm{~g} / \mathrm{m}^{3}$ for AZ-102 Melt 1, and $122.1 \mathrm{~g} / \mathrm{m}^{3}$ for AZ-102 Melt 2, none of which exceed the contract plutonium loading of 2500 grams per cubic meter; and lastly 4) The ratio by weight of the total element of the following isotopes: U-233, U-234, U-235, U-236, U-238, Pu-238, Pu-239, Pu-240, and Pu-241, are reported. The above IHLW glass product, radionuclide compositional data demonstrates that all three glasses pass the ORP Phase B-1 contract criteria.

Identification and quantification of crystalline and non-crystalline phases were completed by using x-ray diffraction (XRD), optical microscopy, and scanning electron microscopy (SEM) on samples given a slow cool down heat treatment which simulated the calculated cooling profile for glass at the centerline of a Hanford HLW canister during filling. No crystals were observed in the C-104 sample by XRD, optical examination, and SEM analysis. XRD analysis combined with optical microscopy and SEM EDS results of the CCC heat-treated AZ-102 Melt 1 glass sample indicated the presence of a 
crystalline phase at approximately 1 to 2 volume percent. An XRD search match analysis of the major peaks found in the XRD pattern of the CCC heat-treated AZ-102 Melt 1 glass sample indicated trevorite $\left(\mathrm{NiFe}_{2} \mathrm{O}_{4}\right)$ and chromite $\left(\mathrm{FeCr}_{2} \mathrm{O}_{4}\right)$ as the most likely spinel crystals. The small amount of crystalline material in the CCC heat-treated AZ-102 Melt 1 glass sample does not significantly alter the leaching resistance of the glass as indicated by the PCT test results. SEM examination of both the C-104 and AZ-102 Melt 1 CCC heat-treated glasses at magnifications up to $5000 \times$ showed homogenous glasses with no evidence of multiple noncrystalline phases.

The ultimate objective for immobilization of the high-level radioactive tank waste is to incorporate and convert the radioactive and hazardous components into a solid waste form that will be chemically durable and meet the conditions for storage in a geologic repository for high-level radioactive waste. This resistance of the waste form to release deleterious environmental components is defined by measuring its chemical durability, i.e. the resistance of the glass to react with the aqueous environment expected in the glass disposal site. However, to mimic the mean temperature, amount and frequency of available ground water, etc. expected in the geologic repository would require a great amount of testing time to be able to detect glass dissolution. Therefore, an accelerated chemical durability test, the Product Consistency Test (PCT) (ASTM C1285-97), is employed to gauge the IHLW glass chemical durability. The glass samples used in the PCT were given a slow cool-down heat treatment, which simulates the cooling profile for glass at the center line of a Hanford standard HLW canister being filled with a waste glass and allowed to cool to ambient temperature. The PCT was run at $90^{\circ} \mathrm{C}$ to determine the normalized release of boron, sodium, lithium, aluminum, and silicon. The Environmental Assessment glass (EA glass) test reference material, standard glass (Jantzen et al. 1993) was included in these tests to provide a reliable baseline of results by which to judge the quality of the PCT results for the C-104 and AZ-102 Melt 1 glasses. The normalized lithium, sodium and boron $90^{\circ} \mathrm{C} \mathrm{PCT}$ release rates for the C-104, AZ-102 Melt 1, and EA glasses are: 1) (for lithium) $0.5 \mathrm{~g} / \mathrm{m}^{2}, 0.4 \mathrm{~g} / \mathrm{m}^{2}$, and $3.75 \mathrm{~g} / \mathrm{m}^{2}$; 2) (for sodium) $0.4 \mathrm{~g} / \mathrm{m}^{2}, 0.4 \mathrm{~g} / \mathrm{m}^{2}$, and $5.1 \mathrm{~g} / \mathrm{m}^{2}$; and 3) (for boron) $0.4 \mathrm{~g} / \mathrm{m}^{2}, 0.3 \mathrm{~g} / \mathrm{m}^{2}$, and $6.9 \mathrm{~g} / \mathrm{m}^{2}$, respectively. More importantly, as the average, normalized elemental release rates are an order of magnitude more durable for $\mathrm{Na}$, and $\mathrm{B}$, and just slightly less than an order of magnitude below for $\mathrm{Li}$ when compared to the reported results of the benchmark EA glass (Jantzen et al., 1993), the ORP Phase B-1 contract criteria were easily met.

Finally, the IHLW product testing results from the C-104 and AZ-102 glasses show that in all cases they meet or exceed ORP Phase B-1 contract specifications for waste loading, chemical composition documentation, radionuclide concentration limitations, and waste form testing (i.e. chemical durability). 


\section{Terms and Abbreviations}

AES

ALO

APEL

ARG-1

ASME

ASTM

$\mathrm{CCC}$

CFR

CMC

CUA

CUF

DI

DIW

DL

DOE

DOE/EM

DOE-RL

DWPF

EA glass

EDS

EPA

EQL

$\mathrm{g}$

GEA

HLRF

HLW

HPGe

HWMA

IAEA

IC

ICP-AES

ICP-MS
Atomic emission spectroscopy

Analytical Laboratory Operations

Applied Process Engineering Laboratory

Analytical Reference Glass-1

American Society of Mechanical Engineers

American Society for Testing and Materials

canister centerline cooling

Code of Federal Regulations

Chemical management center

Catholic University of America

cells unit filter

Deionized

deionized water

detection level

U.S. Department of Energy

U.S. Department of Energy, Office of Environmental Management

U.S. Department of Energy-Richland Operations Office

Defense Waste Processing Facility

Environmental Assessment glass

energy dispersive spectroscopy

Environmental Protection Agency

estimated quantification limit

gram

gamma energy analysis

High-Level Radiochemistry Facility

high level waste

high purity germanium

Hazardous Waste Management Act

International Atomic Energy Agency

Ion chromatography

inductively coupled plasma-atomic emission spectroscopy

inductively coupled plasma-mass spectroscopy 


\begin{tabular}{|c|c|}
\hline ICV & initial calibration verification \\
\hline IHLW & immobilized high-level waste \\
\hline ILAW & immobilized low activity waste \\
\hline $\mathrm{L}$ & liter \\
\hline LAW & low activity waste \\
\hline LCS & Laboratory control standard \\
\hline LEPS & low-energy photon spectrometry \\
\hline LFCM & liquid-fed ceramic melter \\
\hline $\mathrm{MCC}$ & Materials Characterization Center \\
\hline MDA & minimum detectable activity \\
\hline MS & mass spectrometry \\
\hline MSE & mean squared error \\
\hline NIST & National Institute of Standards and Technology \\
\hline NM & not measured \\
\hline NQARD & Nuclear Quality Assurance Requirements and Description \\
\hline NUREG & Nuclear Regulation \\
\hline OCRWM & U.S. DOE Office of Civilian Radioactive Waste Management \\
\hline ORP & Office of River Protection \\
\hline PCB & polychlorinated biphenyls \\
\hline PCT & product consistency test \\
\hline PND & Pacific Northwest Division \\
\hline PNNL & Pacific Northwest National Laboratory \\
\hline QA & Quality Assurance \\
\hline QC & Quality Control \\
\hline QARD & $\begin{array}{l}\text { Quality Assurance Requirements and Description for the Civilian } \\
\text { Radioactive Waste Management Program }\end{array}$ \\
\hline RCRA & Resource Conservation Recovery Act \\
\hline RPD & Relative Percent Difference \\
\hline RPG & Radiochemical Processing Group \\
\hline RPL & Radiochemical Processing Laboratory \\
\hline RPP-WTP & River Protection Project Waste Treatment Plant \\
\hline SAL & Shielded Analytical Laboratory \\
\hline SBMS & Standards Based Management System \\
\hline SEM & Scanning Electron Microscopy \\
\hline SRTC & Savannah River Technology Center \\
\hline
\end{tabular}


STP standard temperature and pressure

SVOA semi-volatile organic analysis

TC total carbon

TCLP Toxicity Characteristic Leaching Procedure

TIC total inorganic carbon

TIMS thermal ionization mass spectroscopy

TOC total organic carbon

TRU transuranic

$\mu \mathrm{m} \quad$ Micron

UST underground storage tank

UTS Universal Treatment Standards

VOA volatile organic analysis

vol\% volume percent

VSL Vitreous State Laboratory

WAPS Waste Acceptance Product Specifications for Vitrified High-Level Waste Forms

WASRD Waste Acceptance System Requirements Document

WCP Waste Form Compliance Plan

WQR Waste Form Qualification Report

wt $\% \quad$ Weight percent

WVDP West Valley Demonstration Project

XRD X-ray diffraction 


\section{Contents}

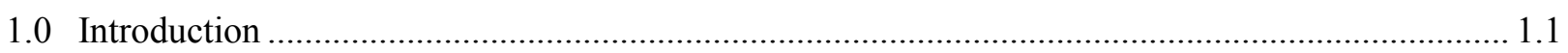

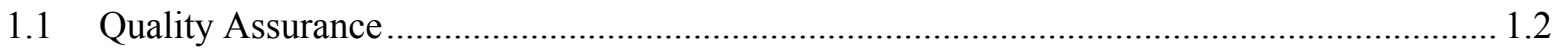

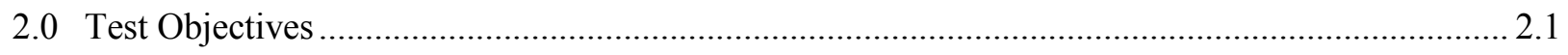

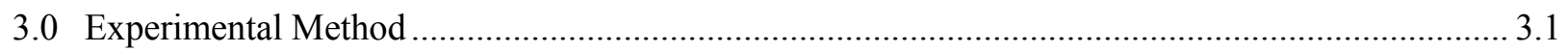

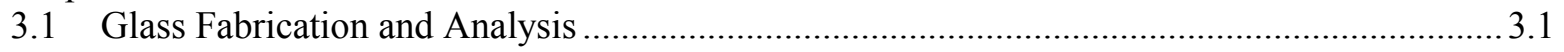

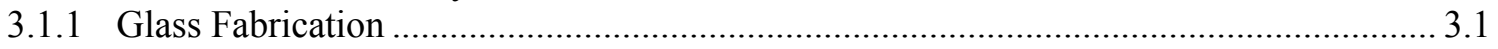

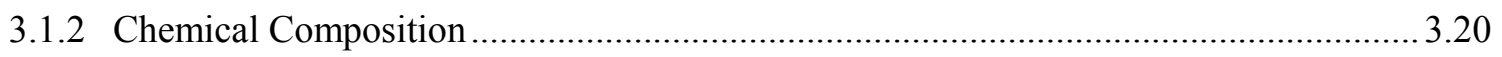

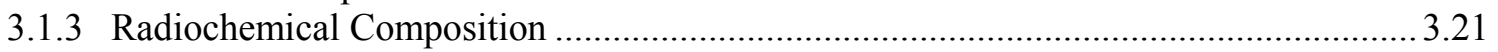

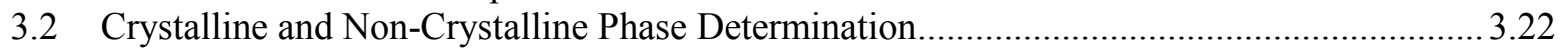

3.3 Release Rate, Product Consistency Testing of HLW Glasses.............................................. 3.24

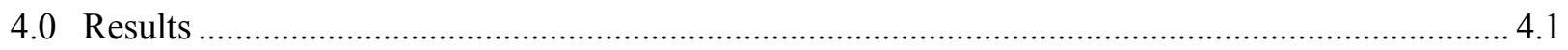

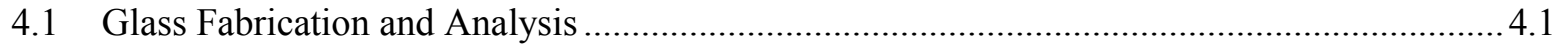

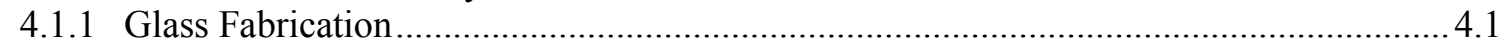

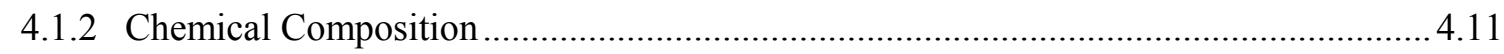

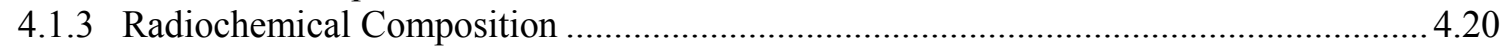

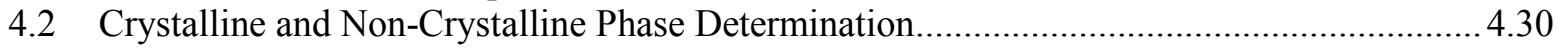

4.3 Release Rate, Product Consistency Testing of HLW Glasses ............................................. 4.35

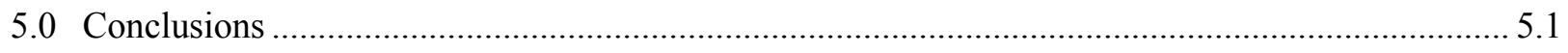

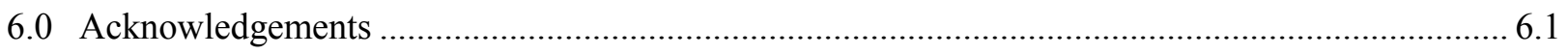

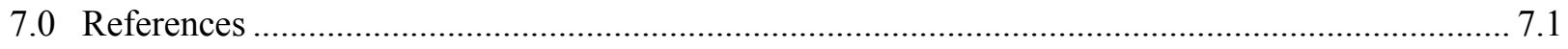

Appendix A: Composited AZ-102 Pretreated Sludge Waste Analysis Data........................................ A.1

Appendix B: Test Instruction: "Preparation of Simulated Sr/TRU Removal Solids for Rheology

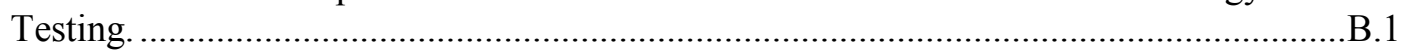

Appendix C: HLW Radioactive Glass Analysis Data ....................................................................

Appendix D: Crystalline and Non-Crystalline Phase Determination Data ......................................... D.1

Appendix E: Release Rate, Modified Product Consistency Test (PCT) Data...................................... 1 


\section{Figures}

Figure 3.1. View of the HLRF gallery looking at the front face of the three hot cells. The lime green color of the windows is from the thick lead glass used for shielding. Two sets of manipulator arms are in front of the two windows in hot cell " $A$ " (foreground), with hot cells " $\mathrm{B}$ " and " $\mathrm{C}$ " only having single sets of manipulators for the smaller hot cells (hot cells A through $\mathrm{C}$ are from right to left)....

Figure 3.2 View through hot cell "A-South" window with high temperature furnace on the left and miscellaneous glass processing equipment on floor of hot cell.

Figure 3.3. The pretreated C-104 tank waste archived sample. This photo gives a good view of the settled waste with the yellow supernate segregated from the solids. Next to the C-104 waste is the empty storage container for the pretreated AZ-102 tank waste, which has a high dose rate and left the glass container darkened where the waste solids had settled.

Figure 3.4. The automated grinder it shown in the center-left of the picture with a sintered aluminum oxide mortar and pestle clamped between the two black metal supports atop the machine. The top metal support is removable so that the pestle can be taken out to remove the crushed sample. A stack of 3-inch diameter stainless steel sieves is in the foreground, jars of processed feed are adjacent to the grinder, and the in-cell video camera is lying on its side next to the poly water dispenser.

Figure 3.5. Samples of C-104 and AZ-102 glass in platinum 10\% rhodium box crucibles covered with lids are placed on the hot hearth plate for the CCC heat treatment. The pedestal that holds the hearth plate will be hydraulically lifted into the heating cavity of the high temperature furnace.....

Figure 3.6. Cutting process for preparation of the AZ-102 CCC heat-treated glass samples showing the beginning cut using a diamond wafering blade.

Figure 3.7. Cutting process for preparation of the AZ-102 CCC heat-treated glass samples showing the final sectioning stage where the manipulator fingers are grasping the section of glass just about to be separated from the original sample.

Figure 3.8. Pictured are the Desensitized Type 304L stainless steel, $22 \mathrm{~mL}$, PCT vessel and lid, a white Teflon gasket which seals the vessel and lid when the assembly is closed and tightened, and the nickel-plated brass, nut and screw vessel tightening assembly.

Figure 4.1. Thick C-104 melter feed sludge, at the bottom of a $4 \mathrm{~L}$ stainless steel beaker, is stirred with a stainless steel stir rod rotated by the hot cell manipulator arm.

Figure 4.2. The C-104 melter feed in large chunks in the Pt10\%Rh crucible after drying on the hot plate. The tip of the stainless steel stir rod is in the foreground above the crucible.

Figure 4.3. The Thermolyne furnace used for drying and calcining the C-104 melter feed. The manipulator arm is holding the furnace door open and the Pt $10 \% \mathrm{Rh}$ crucible with dried feed is inside the furnace cavity. Note the dark stains on the white insulation surrounding the furnace cavity. This is probably from nitrate volatilizing during the drying cycle.

Figure 4.4. Calcined C-104 melter feed in glass jar next to the Pt10\%Rh crucible.

Figure 4.5. Powdered, calcined melter feed is added to a stainless steel funnel to help load it into the crucible in preparation for melting. The funnel is used to evenly distribute the powder into the hot crucible as additional batches of melter feed are added to the melt.

Figure 4.6. The C-104 glass sample is air quenched on a stainless steel tray. Note the box crucible full of molten glass on the left side of the glass pour. 
Figure 4.7. Dried AZ-102 Melt 1 melter feed is being loaded into the Pt10\%Rh crucible using the stainless steel funnel in preparation for calcining. ...

Figure 4.8. Approximately 50 percent of the AZ-102 Melt 1 powdered calcined feed loaded in the $\mathrm{Pt} 10 \% \mathrm{Rh}$ crucible and ready for melting.

Figure 4.9. Molten AZ-102 Melt 2 glass being air quenched on a stainless steel plate.

Figure 4.10. The HLW Process Blank (AZ-102 Melt 1 simulant) glass being poured.

Figure 4.11. The HLW Process Blank (AZ-102 Melt 1 simulant) glass pour just after air quenching on the stainless steel tray.

Figure 4.12. These XRD diffraction patterns for HLW glasses C-104 and AZ-102 Melt 1 shows general amorphous characteristics. The small sharp crystalline peaks superimposed on the AZ-102 Melt 1 CCC heat-treated glass diffraction pattern indicate a small amount of a crystalline phase present in this glass.

Figure 4.13. An optical microscope was used to examine the AZ-102 Melt 1 CCC heat-treated glass sample at a magnification of $200 \times$ using transmitted light. The picture shows cubic crystals that are evenly dispersed with an average size of about 10 microns.

Figure 4.14. SEM photomicrograph, taken at a magnification of $2000 \times$, of the surface of the CCC heat-treated AZ-102 Melt 1 glass thin section. Elemental analysis of a crystal (designated in the picture as "eds01") is provided in Figure 4.15 and of the glass matrix (designated in the picture as "eds02") in Figure 4.16.

Figure 4.15. EDS scan of the area outlined and labeled "eds01" on the crystal (center right) in the photomicrograph of Figure 4.14. Note the high iron and oxygen peaks combined with moderate nickel and low chrome and manganese peaks, all major components of a spinel $\left((\mathrm{Fe}, \mathrm{Ni}, \mathrm{Mn})(\mathrm{Fe}, \mathrm{Cr})_{2} \mathrm{O}_{4}\right)$ crystal. Silicon, aluminum, and zirconium peaks are from the glass matrix or impurities within the crystal structure. The carbon is probably from super glue contamination

Figure 4.16. EDS scan of the area outlined and labeled "eds02"on the glass surface (upper right corner) in the photomicrograph of Figure 4.14. These are typical peaks from the glass matrix of a HLW glass....

Figure D. 1 and D.2 are photos of the surface of the thin section of C-104 CCC heat-treated glass. Figure D.1 is polished to 1200 grit, note the many scratches and the dull appearance of the surface. Figure D.2 is polished with a 6 micron polishing paste and is much smoother, though some scratches are still evident. The surface of Figure D.2 is still not as shiny as most HLW glasses would be with this polish.

Figure D.3. Photo of the surface of C-104 CCC heat-treated glass using reflected light and an optical microscope at 100x. This surface is very rough. The black spots are the pitted areas from the polishing process. A scratch mark is observed as a dashed line going diagonally through the photo. In contrast, look at the smooth surface of Figure D.7 polished in the same manner as this sample.

Figure D.4. The surface of C-104 CCC heat-treated glass at 200x magnification using optical microscopy (reflected light). Black and dark gray areas are pits in the glass caused from grinding and polishing the sample. These pits are not common to polished HLW glasses.

Figure D.5. SEM photomicrograph at 5000x magnification of the surface of C-104 CCC heattreated glass. Both pits and scratches can be observed form the grinding and polishing of this sample. Minor contamination from sample preparation on the sample surface appears as small irregular white and gray spots. No crystals were observed. .....

Figure D.6. Another photomicrograph of C-104 CCC heat-treated glass. Conditions the same as Figure D.5. D.4

Figure D.7. Examination of the surface of AZ-102 CCC heat-treated glass using an optical microscope with magnification of 50x (reflected light). Bright white speckles are 
the crystals in the glass matrix. The gray shadow at the center-right of the picture is an imperfection in the equipment used.

Figure D.8. An optical microscope was used to examine AZ-102 CCC heat-treated glass at a magnification of 200x using transmitted light. Cubic crystals are evenly dispersed and average in size about 10 microns.

Figure D.9. SEM photomicrograph at 500x of the surface of a polished section of the AZ-102 CCC heat-treated glass. The dark background is the glass matrix, the large white shapes are crystals, and the small white specks are contamination from sample preparation. Elemental analysis of the sample is provided in Figures D.10, D.11, and D. 12 .

Figure D.10. SEM photomicrograph taken at 2000x of the surface of AZ-102 CCC heat-treated glass. Elemental analysis of a crystal (eds01) is provided in Figure D.11 and of the glass matrix (eds02) in Figure D.12.

Figure D.11. EDS scan of the area outlined and labeled "eds01"'on the crystal (center right) in the photomicrograph of Figure D.10. Note the high iron and oxygen peaks combined with moderate nickel and low chrome and manganese peaks, all major components of a spinel $\left((\mathrm{Fe}, \mathrm{Ni}, \mathrm{Mn})(\mathrm{Fe}, \mathrm{Cr})_{2} \mathrm{O}_{4}\right)$ crystal. Silicon, aluminum, and zirconium peaks are from the glass matrix or impurities within the crystal structure. The carbon is probably from super glue contamination.

Figure D.12. EDS scan of the area outlined and labeled "eds02"on the glass surface (upper right corner) in the photomicrograph of Figure D.10. These are typical peaks from the glass matrix of a HLW glass.

Figure D.13 and D.14 are SEM photomicrographs of the surface of a polished section of the AZ102 CCC heat-treated glass.

Figure D.15. EDS scan of the area outlined and labeled "eds03"'on the crystal (top right) in the photomicrograph of Figure D.14. Note the high iron and oxygen peaks combined with moderate nickel and low chrome and manganese peaks, all major components of a spinel $\left((\mathrm{Fe}, \mathrm{Ni}, \mathrm{Mn})(\mathrm{Fe}, \mathrm{Cr})_{2} \mathrm{O}_{4}\right)$ crystal. Silicon, aluminum, and zirconium peaks are from the glass matrix or impurities within the crystal structure. The carbon is probably from super glue contamination.

Figure D.16. EDS scan of the area outlined and labeled "eds04"on the glass surface (upper left corner) in the photomicrograph of Figure D.14. These are typical peaks from the glass matrix of a HLW glass

Figure D.17. This is a graph of a search match analysis of the major peaks found using X-ray diffraction of the AZ-102 CCC heat-treated glass. Trevorite and chromite were the two crystal structures that matched the major peaks well. Both crystal structures are typical matches for spinel crystals identified in HLW glasses. 


\section{Tables}

Table 3.1. Composition of C-104 (Envelope D) Pretreated Sludge Waste ........................................... 3.5

Table 3.2. Composition of AZ-102 (Envelope D) Composited Pretreated Sludge Waste ...................... 3.6

Table 3.3. Secondary Waste Compositions - Cs Ion Exchange Eluants..................................................3.7

Table 3.4. Secondary Waste Compositions - Tc Eluant Composite and Sr/TRU Precipitate ................. 3.8

Table 3.5. C-104 and AZ-102 Mineral and Chemical Glass Former Additives..................................... 3.9

Table 3.6. Waste Component Compositions and Batching Ratios to Make up the C-104 Melter Feed ......................................................................................................... 3.10

Table 3.7. C-104 Pretreated Sludge Waste and Secondary Waste Additions to Produce C-104 Melter Feed for 250g of Glass .................................................................................... 3.11

Table 3.8. Grams of Mineral Additives Added to the Waste Slurry to Make 250g of C-104 Glass..... 3.11

Table 3.9. Recipe for HLW AZ-102 Melt 1 Glass Composition from VSL, Using Pretreated

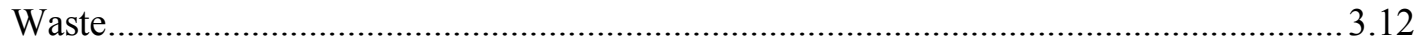

Table 3.10. AZ-102 Pretreated Waste and Secondary Waste Additions to Melter Feed ...................... 3.13

Table 3.11. Grams of Mineral Additives added to the Waste Slurry to Make $118.92 \mathrm{~g}$ of Glass from the AZ-102 Melt 1 Melter Feed ................................................................................. 3.14

Table 3.12. Waste Compositions and Batching Ratios for the AZ-102 Melt 2 Waste Glass............... 3.15

Table 3.13. AZ-102 Melt 2 Pretreated Waste and Secondary Waste Additions to Melter Feed............ 3.16

Table 3.14. Grams of Mineral Additives added to the Waste Slurry to Make up $69.81 \mathrm{~g}$ of Glass from the AZ-102 Melt 2 Melter Feed .................................................................................. 3.16

Table 3.15. AZ-102 Melt 1 Simulant Used to Produce the HLW Process Blank Glass. Note that the AZ-102 Melt 1 Composition has Been Adjusted for the Substitution of Neodymium Oxide for Uranium Oxide on a Molar Basis

Table 3.16. Temperature Profile Line Segments Used as Guidelines for Programming the Del Tech Furnace Controller to Generate the Hanford HLW Canister Centerline Cooling Profile.

Table 4.1. Target, Measured, Adjusted, and Normalized Adjusted Compositions of AZ-102 Melt 1 Radioactive Glass. See Table 4.4 for a compilation of the footnotes for Tables 4.1, 4.2 , and 4.3

Table 4.2. Target, Measured, Adjusted, and Normalized Adjusted Compositions of AZ-102 Melt 2 Radioactive Glass. See Table 4.4 for a compilation of the footnotes for Tables 4.1, 4.2, and 4.3

Table 4.3. Target, Measured, Adjusted, and Normalized Adjusted Compositions of C-104 Radioactive Glass. See Table 4.4 for a compilation of the footnotes for Tables 4.1, 4.2 , and 4.3 .....

Table 4.4. Compilation of the Footnotes for Tables 4.1, 4.2, and 4.3

Table 4.5. Waste Loading/Dilution factors for HLW Waste Glass C-104 ...................................... 4.18

Table 4.6. Waste Loading/Dilution factors for HLW Waste Glass AZ-102 Melt 1............................ 4.19

Table 4.7. Waste Loading/Dilution factors for HLW Waste Glass AZ-102 Melt 2........................... 4.19

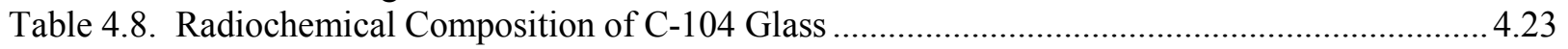

Table 4.9. Radiochemical Composition of AZ-102 Glasses ....................................................... 4.24

Table 4.10. Radioisotopes with Half-Lives Longer than 10 Years and Half-Life Factors Indexed to years 2015 and 3115

Table 4.11. Radioisotopes with Half-Lives Longer than 10 Years Indexed to years 2015 and 3115 for HLW Glass C-104. 4.26

Table 4.12. Radioisotopes with Half-Lives Longer than 10 Years Indexed to years 2015 and 3115 for HLW Glass AZ-102 Melt 1. 
Table 4.13. Radioisotopes with Half-Lives Longer than 10 Years Indexed to years 2015 and 3115 for HLW Glass AZ-102 Melt 2.

Table 4.14. ICP-MS Data Providing the ${ }^{99} \mathrm{Tc}$, Uranium Isotopic, ${ }^{237} \mathrm{~Np}$, ${ }^{239} \mathrm{Pu}$, and ${ }^{240} \mathrm{Pu}$ Glass

Content for Each of the HLW Glasses.................................................................... 4.28

Table 4.15. The Total and Fissile Uranium and Plutonium (U-233, U-235, Pu-239, and Pu-241) Content of Each Glass Waste Type in Grams for a Cubic Meter of Glass ....................... 4.28

Table 4.16. The Concentration of Plutonium in Grams per Cubic Meter for Each Hanford HLW Canister.

Table 4.17. The Ratio by Weight to Total Weight of the Element for the Following Isotopes: U-233, U-234, U-235, U-236, and U-238

Table 4.18. The Ratio by Weight of the Total Element of the Following Isotopes: Pu-238, Pu-239, $\mathrm{Pu}-240$, and Pu-241. Pu-242 is Not Reported As It Was Used As A Tracer For The Plutonium Separations ....

Table 4.19. Temperature Profile Line Segments for the Del Tech Furnace Controller to Generate the Hanford HLW Canister Centerline Cooling Profile...

Table 4.20. Comparison of $90^{\circ} \mathrm{C}$ PCT Results from the Environmental Assessment (EA) Glass Round Robin (Jantzen, et al. 1993) with the Equivalent Values for the $90^{\circ} \mathrm{C}$ PCT in this Report.

Table 4.21. Average 7-Day $90^{\circ} \mathrm{C}$ PCT Normalized Mass Loss Data of Radioactive IHLW Glasses ......

Table 4.22. Comparison of Average 7-Day $90^{\circ} \mathrm{C}$ PCT Normalized Mass Loss Data Between VSL Non-radioactive Simulant Glasses and Actual Radioactive HLW Glass Counterparts from this Study. Environmental Assessment (EA) Data is Provided for Comparison Purposes.

Table A.1. ICPAES Data Report for AZ-102 Washed Solids Sample \#1 ......................................... A.2

Table A.2. ICPAES Data Report for AZ-102 Washed Solids Sample \#2 .............................................3

Table C.1. ICP-AES Data for HLW Glass C-104-Sodium Peroxide Fusion in a Zirconium Crucible.

Table C.2. ICP-AES Data for HLW Glass C-104-Potassium Hydroxide Fusion in a Nickel Crucible. C. 3

Table C.3. ICP-AES Data for HLW Glass AZ-102 Melt 1-Sodium Peroxide Fusion in a Zirconium Crucible...

Table C.4. ICP-AES Data for HLW Glass AZ-102 Melt 1-Potassium Hydroxide Fusion in a Nickel Crucible.

Table C.5. ICP-AES Data for HLW Glass AZ-102 Melt 2-Sodium Peroxide Fusion in a

Zirconium Crucible.

Table C.6. ICP-AES Data for HLW Glass AZ-102 Melt 2-Potassium Hydroxide Fusion in a Nickel Crucible

Table C.7. ICP-AES Data for ARG-1 Reference Glass-Sodium Peroxide Fusion in a Zirconium Crucible.

Table C.8. ICP-AES Data for ARG-1 Reference Glass-Potassium Hydroxide Fusion in a Nickel Crucible.

Table C.9. Radioactive HLW Glasses C-104 and AZ-102 Melt 1 Radiochemical Composition Data by GEATable C.10. Radioactive HLW Glasses C-104 and AZ-102 Melt 1 Radiochemical Composition Data by GEA - Detection Limits C. 10

Table C.10. Radioactive HLW Glasses C-104 and AZ-102 Melt 1 Radiochemical Composition Data by GEA - Detection Limits

Table C.11. Radioactive HLW Glasses C-104 and AZ-102 Melt 1 Radiochemical Composition Data - $\mathrm{Sr}$ and $\mathrm{Pu}$ Isotopes.

Table C.12. Radioactive HLW Glasses C-104 and AZ-102 Melt 1 Radiochemical Composition Data - Total Uranium by Kinetic Phosphorescence. 
Table C.13. Radioactive HLW Glasses C-104, AZ-102 Melt 1, and AZ-102 Melt 2 Radiochemical Composition Data by ICP-MS (Tc-99, U-233, U-234) C. 14

Table C.14. Radioactive HLW Glasses C-104, AZ-102 Melt 1, and AZ-102 Melt 2 Radiochemical Composition Data by ICP-MS (U-235, U-236, U-238)

Table C.15. Radioactive HLW Glasses C-104, AZ-102 Melt 1, and AZ-102 Melt 2 Radiochemical Composition Data by ICP-MS (Total U, Np-237, Pu-239, Pu-240) ....... C.16

Table E.1. Product Consistency Testing (PCT) Leachate Analysis Data for Samples C-104-CCC90-1 to 90-3 and for AZ-102-CCC-90-1 and 2

Table E.2. Product Consistency Testing (PCT) Leachate Analysis Data for Samples AZ-102-903, EA-90-1 to 90-3, and Blank-90-1 .E.3

Table E.3. Product Consistency Testing (PCT) Leachate Analysis Data for Samples Blank-90-2 and Blank 90-3 


\subsection{Introduction}

The U.S. Department of Energy (DOE) Office of River Protection (ORP) has acquired Hanford tank waste treatment services at a demonstration scale. The River Protection Project Waste Treatment Plant (RPP-WTP) team is responsible for producing an immobilized (vitrified) high-level waste (IHLW) waste form. Pacific Northwest National Laboratory, hereafter referred to as PNNL, has been contracted to produce and test a vitrified IHLW waste form from two Envelope D high-level waste (HLW) samples previously supplied to the RPP-WTP project by DOE.

The U.S. Department of Energy currently has radioactive waste stored in underground storage tanks (USTs) at the Hanford site in southeastern Washington. One sludge sample each was taken from two of the USTs. The particular tanks of interest (241-C-104 and 241-AZ-102) are of double-shell construction and are 1-million gallon in capacity. The two tank sludge samples were processed through pretreatment chemical washing and leaching processes, and the pretreated sludges were converted to high-level waste (HLW) glass after flowsheet quantities of secondary wastes, i.e. Sr/TRU precipitate and Cs and Tc ion exchange eluants, generated from LAW supernatant pretreatment unit operations were added. Both sludge samples were processed through the following unit operations to simulate the RPP-WTP project flowsheet: 1) initial characterization; 2) washing; 3) leaching; and 4) filtration in a crossflow filtration system (Brooks et al., 2000a) (Brooks et al., 2000b). Additional washing/leaching and solubility versus temperature studies were completed on a small subsample of C-104 tank sludge that was not vitrified (Lumetta et al., 2000). In addition to the initial characterization of the C-104 tank sludge, subsamples were extensively analyzed for inorganic, radiochemical, and organic constituents (Evans et al., 2000 and Fiskum et al., 2000). Physical and rheological testing of the C-104 and AZ-102 pretreated sludge and melter feeds has also been completed and reported in a separate document (Bredt et al., 2000).

The primary objective for vitrifying the two Envelope D (Tank C-104 and Tank AZ-102) pretreated HLW sludge samples was to characterize the glass produced from the crucible melts. Testing of the waste glasses produced from actual tank waste will also show compliance with the RPP-WTP contractual requirements such as chemical and radionuclide reporting, product loading, and dangerous waste limitations and organic content in the glasses.

The scope of this work was divided into 8 work elements: 1) Glass Fabrication, 2) Chemical Composition, 3) Radiochemical Composition, 4) Crystalline and Non-crystalline Phase Determination, 5) Release Rate (PCT), 6) Dangerous Waste Limitations - Toxicity Characteristic Leaching Procedure (TCLP), 7) Total volatile organic and semi-volatile organic analyses (VOA and SVOA), and 8) Regulatory Testing. This report will discuss the results for work elements 1 through 5. Results for work elements 6 through 8, i.e. VOA, SVOA, dioxins, furans, PCBs, and total cyanide and sulfide analyses, are presented in a different report (Goheen et al., WTP-RPT-010).

Two pretreated tank sludge samples, high-level wastes (241-C-104 and 241-AZ-102) along with a HLW process simulant (know as the HLW Process Blank) were prepared as melter feeds for vitrification. Due to scheduling constraints and small initial sample size of the pretreated tank 241-AZ-102 sludge, this sample was divided into two samples that were vitrified separately (i.e. AZ-102, Melt 1 and AZ-102, Melt 2). The analyzed compositions of the pretreated C-104 and AZ-102 wastes were used by Catholic University of America's (CUA) Vitreous State Laboratory (VSL) to calculate the target glass composition. 


\subsection{Quality Assurance}

This work was performed in the Pacific Northwest National Laboratory (PNNL) Radiological Processing Laboratory (RPL), Building 325 and Building 326 in Richland, Washington. To provide the River Protection Project-Waste Treatment Plant (RPP-WTP) project with quality products and services, PNNL established and implemented a quality assurance implementation plan for the RPP-WTP project titled "BNFL Phase B-1 Support, Quality Assurance Planning Document," document number BNFL-QAPjP, Rev. 1. The Feed Preparation and Glass Fabrication work was conducted under the quality requirements of the Standards-Based Management System (SBMS) as delineated in Section 4.3 and 4.4.1 of BNFL-QAPjP, Rev. 1. The rest of the work elements related to glass product testing contained in this report were conducted to meet the quality requirements of DOE/RW-0333P, "Quality Assurance Requirements and Description for the Civilian Radioactive Waste Management Program" as delineated in Section 4.3 and 4.4.1 of BNFL-QAPjP, Rev. 1.

The work and results, specifically related to glass product testing, reported herein were conducted under the quality requirements of the Nuclear Quality Assurance Requirements and Description (NQARD) Manual as delineated in Section 4.2, 4.3 and 4.4 of BNFL-QAPjP, Rev. 0 and Section 4.3 and 4.4 of BNFL-QAPJP, Rev. 1. Specific NQARD procedures applied to this work included: Indoctrination and Training; General Hand Calculations; Purchase Requisitions; Obtaining Services; Document Control; Procedure and Instruction Change Control and Change Request; Identification and Control of Test Materials (Testing and Analysis); Test Planning, Performance, and Evaluation; Calibration Control System; Handling, Storing, and Shipping; Inspection and Testing Status and Tagging; Nonconformance Reports; Deficiency Reports; Trend Analysis; Corrective Action; Records System; Laboratory Record Books; and Internal Audits. 


\subsection{Test Objectives}

This work addresses RPP-WTP contract requirements to demonstrate the contractor's ability to satisfy the immobilized high-level waste (IHLW) product requirements (specification 1 of the RPP-WTP Phase B-1 contract) with samples of pretreated HLW and secondary waste products.

\section{Test Objectives:}

The primary objective for vitrifying the HLW sample is to generate a glass product for subsequent product testing. Testing will seek to demonstrate the RPP-WTP projects ability to satisfy the product requirements concerning:

- Chemical and radionuclide reporting.

- Product loading.

- Identification and quantification of crystalline and non-crystalline phases.

- Product consistency.

- Quantification of total sulfide and cyanide.

\section{Success Criteria:}

The primary success criteria are associated with the product requirements:

- The IHLW product for disposal in the proposed geologic repository will be a vitrified borosilicate glass waste form.

- Identification and quantification of those chemical constituents present at concentrations greater than $0.5 \mathrm{wt} \%$, consistent with the Waste Acceptance Product Specifications for Vitrified High Level Waste Forms (WAPS), DOE/EM-0093, specifications 1.1 and 3.14. [Note: WAPS specification 1.1 reads: "Chemical Specification, The waste form is borosilicate waste glass. Subspecification 1.1.1 titled Chemical Composition Projections reads: In the WQR, the Producer shall project the chemical composition, identify crystalline phases expected to be present, and project the amount of each crystalline phase, for each waste type. The method to obtain the required data shall be provided in the WQR. Waste form compositions not available for reporting in the initial WQR shall be included in an addendum to the WQR. Sub-specification 1.1.2 titled Chemical Composition During Production reads: In the Production Records, the Producer shall report the oxide composition of the waste form. The reported composition shall include all elements, excluding oxygen, present in concentrations greater than 0.5 percent by weight of the glass, for each waste type. The Producer shall describe the method to be used for compliance in the WCP. An estimate of the error of the reported composition and the basis for the estimate shall be reported in the WQR." and WAPS specification 3.14 reads: "Concentration of Plutonium in Each Canister Specification, The concentration of plutonium in each HLW standard canister shall be less than 2,500 grams/cubic meter."]

- Identification and quantification of radionuclides consistent with WAPS, specifications 1.2 and 1.6. [Note: WAPS specification 1.2 reads: "The Producer shall report the inventory of radionuclides (in Curies) that have half-lives longer than 10 years and that are, or will be, present in concentrations greater than 0.05 percent of the total radioactive inventory for each waste type, indexed to the years 2015 and 3115.; Sub-specification 1.2.1 titled Radionuclide Inventory Projections reads: The Producer shall provide in the WQR estimates of the total quantities of individual radionuclides to be shipped to the repository, for each waste type. The Producer shall also report the upper limit of these radionuclides for any canistered waste form, and an average 
calculated radionuclide inventory per canister for each waste type. The method to be used to obtain the required data shall be described by the Producer in the WCP. The data shall be provided in the WQR. Radionuclide inventory estimates not available for reporting in the initial WQR shall be included in an addendum to the WQR.; Sub-specification 1.2.2 Radionuclide Inventory During Production reads: The Producer shall provide in the Production Records estimates of the inventories of individual reportable radionuclides for each canister and for each waste type. The Producer shall also report the estimated error of these estimates in the WQR." and WAPS specification 1.6 reads: "IAEA Safeguards Reporting for HLW Specification: The Producer shall report the following in the production records: (1) The total and fissile uranium and plutonium content of each canister in grams. (2) The concentration of plutonium in grams per cubic meter for each canister. (3) The ratio by weight of the total element of the following isotopes: U-233, U-234, U-235, U-236, U-238, Pu-238, Pu-239, Pu-240, Pu-241, and Pu-242.”

- The product loading shall be consistent with the requirements delineated in the RPP-WTP contract, specification 1.2.2.1.6. [Note: The ORP Contract Specification 1, Immobilized HighLevel Waste, section 1.2.2.1.6, Product Loading, states: "Loading of non-volatile components in Envelope D, and, if directed by DOE, entrained solids after washing in accordance with Specification 12, Number of HLW Canister Per Batch of Waste Envelope D, shall be achieved, such that, the concentration of at least one of the waste components or waste component combinations in Table TS-1.1 Minimum Component Limits in HLW Glass exceeds its minimum weight percent in HLW glass as identified in Table TS-1.1 (e.g. for a high-iron waste the Contractor shall incorporate at least 12.5 weight percent iron oxide from the waste into the glass). The product loading shall not cause the limits in any other requirement of this specification to be violated. Product waste loading shall be calculated on an average basis for each batch transfer of Waste Envelope D. The waste loading may be adjusted downward if necessary to comply with Universal Treatment Standards (UTS) leaching requirements."

- Identification and quantification of crystalline and non-crystalline phases consistent with WAPS specification 1.1.1. [Note: WAPS specification 1.1.1 titled Chemical Composition Projections reads: "In the WQR, the Producer shall project the chemical composition, identify crystalline phases expected to be present, and project the amount of each crystalline phase, for each waste type. The method to obtain the required data shall be provided in the WQR. Waste form compositions not available for reporting in the initial WQR shall be included in an addendum to the WQR."

- The normalized release rates of lithium, sodium, and boron shall satisfy the requirements delineated in WAPS specification 1.3.1. [Note: WAPS specification 1.3.1 titled Acceptance Criterion reads: The consistency of the waste form shall be demonstrated using the Product Consistency Test (PCT) [3]. For acceptance, the mean concentrations of lithium, sodium and boron in the leachate, after normalizing for the concentrations in the glass, shall each be less than those of the benchmark glass described in the Environmental Assessment for selection of the DWPF waste form [4]. The measured or projected mean PCT results for lithium, sodium, and boron shall be provided in the Production Records. The Producer shall define the statistical significance of the reported data in the WQR. One acceptable method of demonstrating that the acceptance criterion is met, would be to ensure that the mean PCT results for each waste type are at least two standard deviations below the mean PCT results of the EA glass." 


\subsection{Experimental Method}

\subsection{Glass Fabrication and Analysis}

Two pretreated high-level waste tank sludge samples (C-104 and AZ-102) along with a HLW process simulant (know as the HLW Process Blank and is a simulant of the AZ-102 Melt 1 sample) were prepared as melter feeds for vitrification. Due to scheduling constraints and small initial sample size of the pretreated tank AZ-102 sludge, this sample was divided into two samples that were vitrified separately (i.e. AZ-102, Melt 1 and AZ-102, Melt 2) so that physical and rheological testing could be completed in parallel with the vitrification activities. Approximately half of the pretreated AZ-102 tank sludge was processed through physical and rheological testing and was then vitrified as AZ-102, Melt 2. There was enough pretreated C-104 tank sludge to complete both the physical and rheological and vitrification and product testing scopes of work in parallel. The analyzed compositions of the pretreated C-104 and AZ-102 wastes were used by Catholic University of America's (CUA) Vitreous State Laboratory (VSL) to determine the target glass compositions that were provided to PNNL.

\subsubsection{Glass Fabrication}

High-level waste (HLW) currently stored in underground tanks at Hanford will be treated for offsite, geologic disposal. Treatment will involve physical and chemical separations (pretreatment) to produce a small volume HLW waste and a larger volume low activity waste (LAW) product before either is immobilized in a glass. Intermediate secondary waste products (radionuclide rich streams, i.e. Cs and Tc ion exchange eluants and Sr/TRU precipitates) generated from the physical and chemical separations performed on low activity waste (LAW) will be added to the HLW before vitrification. The HLW product must satisfy a number of performance requirements for it to be accepted for disposal.

The primary objective for vitrifying the Envelope D waste samples is to characterize the glass produced from the crucible melts for Waste Acceptance Product Specifications (WAPS), regulatory, and de-listing purposes. Testing of the waste glasses produced from actual tank waste will also show compliance with the Office of River Protection contractual requirements such as chemical and radionuclide reporting, product loading, and organic content in the glasses. Results from the glass organic and regulatory testing, i.e. VOA, SVOA, dioxins, furans, PCBs, and total cyanide and sulfide analyses are reported in a separate document (Goheen et al., WTP-RPT-010).

The pretreated high-level waste sludges were processed and vitrified in the Radiochemical Processing Laboratory (RPL - also known as the 325 Building) in the High Level Radioactive Facility (HLRF) in the three hot cells (see Figures 3.1 and 3.2). The HLW Process Blank, simulant of the AZ-102 Melt 1 sample, was prepared in the Glass Development Laboratory at the Applied Process Engineering Laboratory (APEL) and then melted in the same high temperature furnace in hot cell "A" of the HLRF used to melt the actual radioactive glasses. 


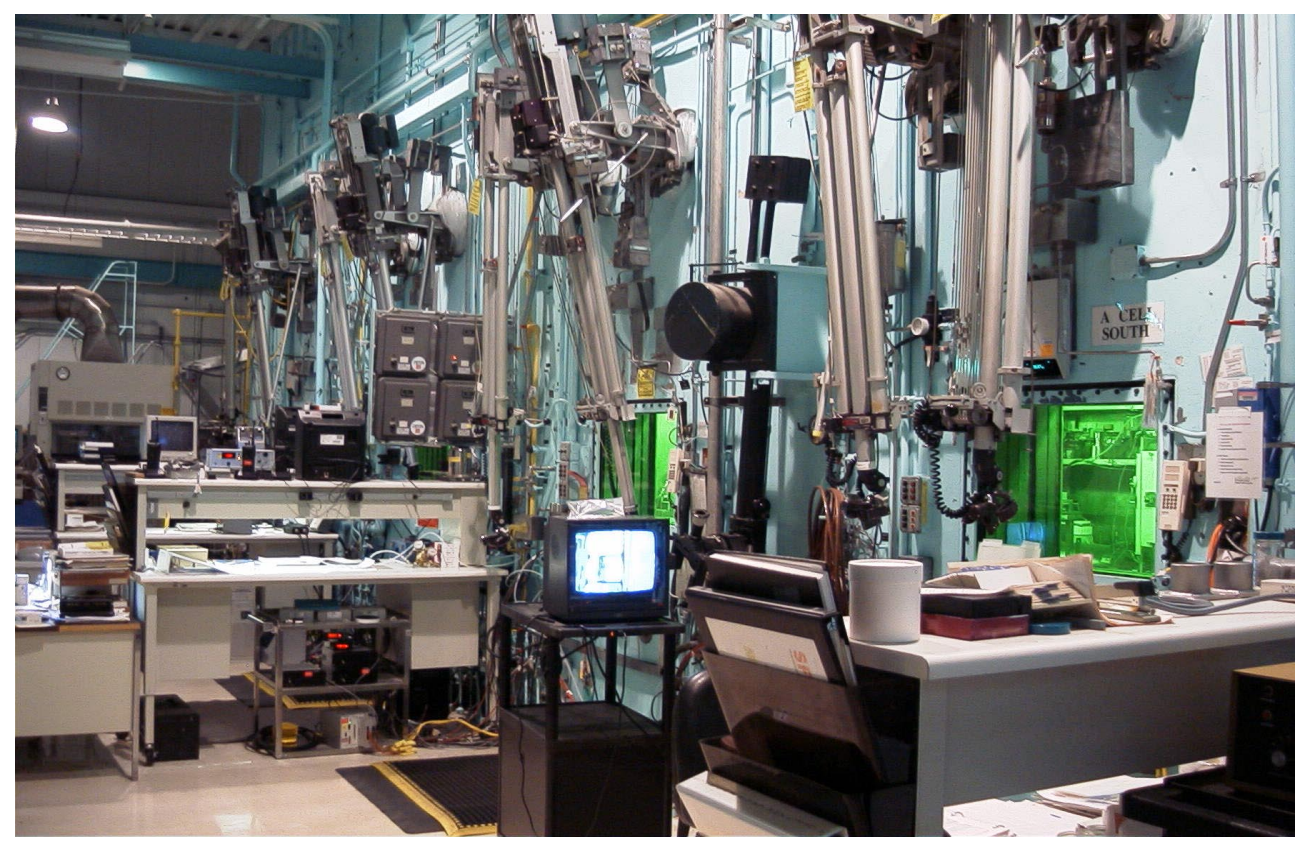

Figure 3.1. View of the HLRF gallery looking at the front face of the three hot cells. The lime green color of the windows is from the thick lead glass used for shielding. Two sets of manipulator arms are in front of the two windows in hot cell " $A$ " (foreground), with hot cells " $B$ " and "C" only having single sets of manipulators for the smaller hot cells (hot cells $A$ through $\mathrm{C}$ are from right to left).

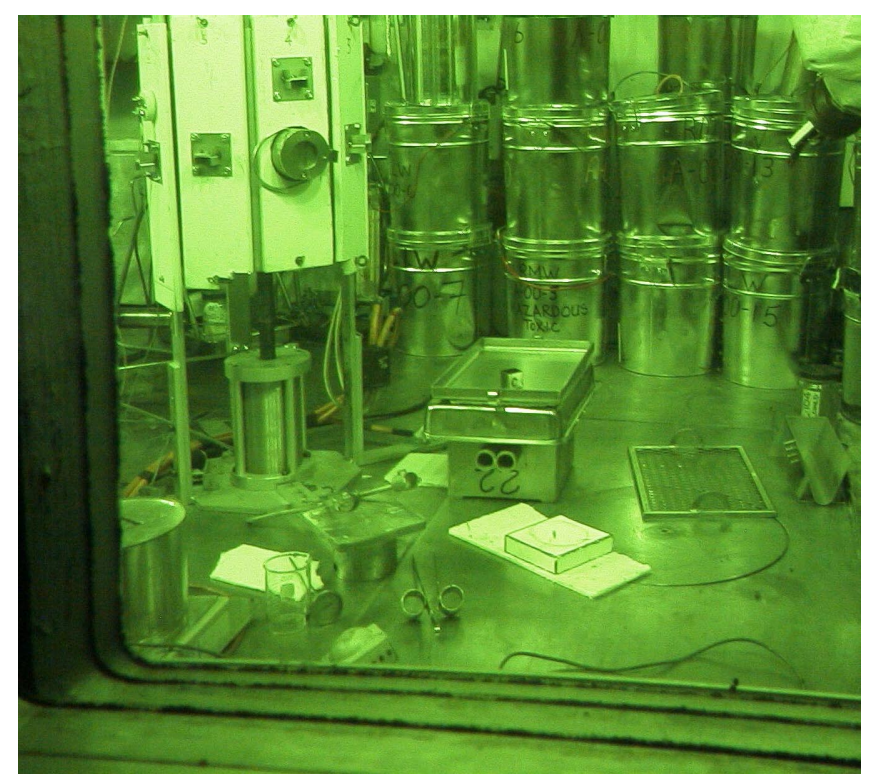

Figure 3.2 View through hot cell "A-South" window with high temperature furnace on the left and miscellaneous glass processing equipment on floor of hot cell. 


\section{HLW Glass Melter Feed Components}

The two tank sludge samples were processed through pretreatment chemical washing and leaching processes, and the pretreated sludges were converted to high-level waste (HLW) glass after flowsheet quantities of secondary wastes were added. The intermediate secondary waste products added (radionuclide rich streams, i.e. Cs and Tc ion exchange eluants and Sr/TRU precipitates) were generated from the physical and chemical separations performed on low activity waste (i.e. AW-101 and AN-107 supernatants).

\section{Tank C-104 and AZ-102 HLW Pretreated Sludge}

Tank C-104 and AZ-102 HLW pretreated sludge waste (the primary melter feed waste constituent) compositions are given in Tables 3.1 and 3.2, respectively. The C-104 tank sludge sample was processed through the following unit operations to simulate the RPP-WTP project flowsheet: 1) washing; 2) leaching (leaching was done external to the CUF unit); and 3) filtration in the cell unit filter (CUF) system which was operated in HLRF hot cell "A" (Brooks et al., 2000a). The AZ-102 tank sludge sample was processed through the same unit operations in the CUF system (Brooks et al., 2000b).

Due to the small initial sample size of the pretreated tank AZ-102 sludge processed, the CUF unit was rinsed with water to retrieve all available pretreated AZ-102 sludge. Only the initial pretreated tank AZ-102 sludge processed through the CUF unit had analytical data and since both samples of pretreated AZ-102 sludge were needed to complete the scope of work for both the physical and rheological and the vitrification and glass product testing, analytical data was needed on the combined pretreated AZ-102 sludge waste. Before combining the pretreated AZ-102 sludge wastes, $214.1 \mathrm{~g}$ of standing liquid (assumed to be water) was removed from the "AZ-102 Washed Solids First Rinse" (sample rinsed from the CUF unit) sample using a peristaltic pump. About $80 \mathrm{~g}$ of the supernate from the "AZ-102 Final Sample" (sample process through the CUF unit) was also removed and stored in a clean container and was eventually used to rinse and clean the two containers and added back to the combined pretreated AZ-102 sludge waste composite. Both AZ-102 pretreated sludge wastes were emptied into a 2 L stainless steel vessel, stirred vigorously with a magnetic stir bar on a stir plate, and sampled for duplicate ICP-AES analysis. The two samples were first measured to determine the weight percent total solids ( 9.49 and 9.58\%) of the composite pretreated AZ-102 sludge waste. The dried duplicate samples were then analyzed for cation composition only (analyses are provided in Appendix A). The analytical values for the anions were calculated by multiplying the original value by the factor $624 / 954=0.654$ because during the compositing approximately $330 \mathrm{~g}$ of non-anion containing solids slurry was incorporated into the composite. The radiochemical analysis values were not changed as the original values were analyzed on a dry solids basis (see Table 3.2 for the final pretreated AZ-102 sludge waste analysis).

\section{$\underline{\text { Secondary Waste: Cs Ion Exchange Eluant }}$}

The Cs ion exchange eluant secondary waste compositions are shown in Table 3.3 and were generated by removal of ${ }^{137} \mathrm{Cs}$ by ion exchange from the AW-101 LAW supernatant (Kurath et al. 2000a) and by removal of ${ }^{137} \mathrm{Cs}$ by ion exchange from the AN-107 LAW supernatant (Kurath et al. 2000b). The various Cs eluants were received in five glass containers. The Cs eluants were combined into a $2 \mathrm{~L}$ stainless steel container; the solution was reconstituted with $34.3 \mathrm{~g}$ of deionized water to restore it to original volume, and mixed on a stir plate with a Teflon stir bar. The Cs ion exchange eluant composite solution was used for all C-104 and AZ-102 melter feeds. The average composition for the Cs ion exchange eluant composite is also found in Table 3.3. 
Total Cs was determined from the Cs-137 analytical values determined by GEA and from the isotopic ratios determined using thermal ionization mass spectroscopy (TIMS). This determination was only performed on the feed but the chemical behavior of all of the Cs isotopes should be the same. For AW-101, the Cs- 137 to total Cs mole ratio was 0.2465 and for AN-107, the Cs- 137 to total Cs mole ratio was 0.2455 . Hence total cesium (i.e. all isotopes of cesium) can be determined from the Cs-137 activity. The cesium eluant is a composite of $64 \mathrm{vol} \%$ from AW-101 and $36 \mathrm{vol} \%$ from AN-107 giving an average Cs-137 to total Cs mole ratio of 0.2461 . The activity of Cs- 137 is $8.7 \mathrm{H} 10^{7} \mu \mathrm{Ci} / \mathrm{g}$, so an average activity of $587.6 \mu \mathrm{Ci} / \mathrm{g}$ for the Cs eluant is equivalent to $6.75 \mathrm{ppm} \mathrm{Cs}-137$ and the total cesium in the eluant is $6.75 / 0.2461=27.4 \mathrm{ppm}$. The Cs-137 activity in the HLW glasses is calculated to be $1280 \mu \mathrm{Ci} / \mathrm{g}$ for $\mathrm{C}-104$ and $847 \mu \mathrm{Ci} / \mathrm{g}$ for AZ-102. Thus the cesium concentration for these glasses is $1280 / 587.6 \mathrm{H} 27.4$ $\mathrm{ppm}=59.7 \mathrm{ppm}$ for $\mathrm{C}-104$ and 847/587.6 H27.4 ppm $=39.5 \mathrm{ppm}$ for AZ-102.

\section{Secondary Waste: Tc Ion Exchange Eluant and Sr/TRU Precipitate Solids}

The Tc ion exchange eluant and the Sr/TRU precipitate solids secondary waste compositions are shown in Table 3.4. The Tc ion exchange eluants were generated by removal of ${ }^{99} \mathrm{Tc}$ by ion exchange from the AW-101 LAW supernatant (Blanchard et al. 1999) and by removal of ${ }^{99} \mathrm{Tc}$ by ion exchange from the AN-107 LAW supernatant (Blanchard et al. 2000). The Sr/TRU precipitate solids were generated by removal of Sr/TRU by precipitation and ultrafiltration to remove entrained solids and the Sr/TRU precipitate from the AN-107 LAW supernatant (Hallen et al. 2000).

Not enough actual Sr/TRU precipitate solids were generated by treating the AN-107 LAW supernatant to match the flowsheet compositional needs for the C-104, AZ-102 Melt 1, and AZ-102 Melt 2 melter feeds. Simulated Sr/TRU precipitate solids were produced, PNNL Test Instruction number BNFL-TI-29953-082, Rev. 0 titled "Preparation of simulated Sr/TRU Removal Solids for Rheology Testing", to provide the necessary balance to produce all three melter feeds (see Appendix B for the referenced PNNL Test Instruction).

\section{$\underline{\text { RPP-WTP Glass Former Minerals }}$}

The glass former minerals used to adjust the waste concentration in the melter feeds and provide the target glass composition are given in Table 3.5. These minerals were sent as dry powder samples from chemical companies that are potential RPP-WTP suppliers. The chemicals were received from each vendor in the condition that would be used to prepare the raw chemical for the vitrification plant at Hanford. The glass formers were combined and processed for each melter feed in the following manner. Each mineral component was weighed on a balance capable of accurately measuring to $10 \mathrm{mg}$. The combined mineral additives for each melter feed were mixed for several minutes in an agate milling chamber. The exact amount of the mineral batch needed to combine with the waste was then weighed out from the blended minerals and mixed with the pretreated sludge and secondary wastes to prepare the various melter feeds.

The current reference chemical additives used to formulate the RPP-WTP glasses (both HLW and LAW) are silica, sucrose, ferric oxide, magnesium silicate, titanium oxide, zircon sand, lithium carbonate, zinc oxide, aluminum silicate, boric acid, calcium silicate, and potentially sodium carbonate. The current reference chemical additives for the RPP-WTP used to formulate the HLW glass are boric acid, lithium carbonate, sodium carbonate, silica, zinc oxide, and sucrose. 
Table 3.2. Composition of AZ-102 (Envelope D) Composited Pretreated Sludge Waste

\begin{tabular}{|c|c|c|c|c|c|}
\hline \multicolumn{6}{|c|}{ Inorganic Analytes: Average Washed Solids } \\
\hline Analyte & $\mu \mathrm{g} / \mathrm{g}$ dry solids & Analyte & $\mu \mathrm{g} / \mathrm{g}$ dry solids & Analyte & $\mu \mathrm{g} / \mathrm{g}$ dry solids \\
\hline $\mathrm{Ag}$ & 442 & $\mathrm{Hg}$ & 0 & $\mathrm{Rh}$ & 0 \\
\hline $\mathrm{Al}$ & 101600 & $\mathrm{~K}^{\mathrm{a}}$ & 0 & $\mathrm{Ru}$ & 0 \\
\hline $\mathrm{B}$ & 63 & $\mathrm{La}$ & 6353 & $\mathrm{Se}$ & 0 \\
\hline $\mathrm{Ba}$ & 842 & $\mathrm{Li}$ & 0 & $\mathrm{Si}$ & 7368 \\
\hline $\mathrm{Be}$ & 24 & $\mathrm{Mg}$ & 1805 & Sn & 3225 \\
\hline $\mathrm{Bi}$ & 0 & $\mathrm{Mn}$ & 5045 & $\mathrm{Sr}$ & 474 \\
\hline $\mathrm{Ca}$ & 8258 & Mo & 0 & $\mathrm{Ta}$ & 0 \\
\hline $\mathrm{Cd}$ & 30575 & $\mathrm{Na}^{\mathrm{b}}$ & 47700 & $\mathrm{Th}$ & 0 \\
\hline $\mathrm{Ce}$ & 1223 & $\mathrm{Nd}$ & 4477 & $\mathrm{Ti}$ & 160 \\
\hline Co & 140 & $\mathrm{Ni}^{\mathrm{a}}$ & 15000 & U (ICP) & 35575 \\
\hline $\mathrm{Cr}$ & 1515 & $\mathrm{P}$ & 4985 & U (laser-fluor.) & NM \\
\hline $\mathrm{Cu}$ & 506 & $\mathrm{~Pb}$ & 2035 & $\mathrm{~V}$ & 51 \\
\hline Dy & 0 & $\mathrm{Pd}$ & 0 & $\mathrm{Y}$ & 287 \\
\hline $\mathrm{Eu}$ & 0 & $\operatorname{Pr}$ & 0 & $\mathrm{Zn}$ & 780 \\
\hline $\mathrm{Fe}$ & 210250 & $\mathrm{Pt}$ & 0 & $\mathrm{Zr}^{\mathrm{b}}$ & 27200 \\
\hline \multicolumn{6}{|c|}{ Ion Chromatography Analytes: Average Washed Solids } \\
\hline Analyte & $\mu \mathrm{g} / \mathrm{g}$ dry solids & Analyte & $\mu \mathrm{g} / \mathrm{g}$ dry solids & Analyte & $\mu \mathrm{g} / \mathrm{g}$ dry solids \\
\hline TIC & 2870 & $\mathrm{Br}$ & $<260$ & $\mathrm{SO}_{4}{ }^{\mathrm{c}}$ & 451 \\
\hline TOC & 2750 & $\mathrm{I}^{\mathrm{c}}$ & 13 & $\mathrm{C}_{2} \mathrm{O}_{4}$ & $<530$ \\
\hline $\mathrm{TC}$ & 5620 & $\mathrm{NO}_{2}$ & $<530$ & $\mathrm{CN}$ & 8.3 \\
\hline $\mathrm{F}^{\mathrm{c}}$ & 196 & $\mathrm{NO}_{3}$ & $<530$ & $\mathrm{NH}_{3}$ & NM \\
\hline $\mathrm{Cl}^{\mathrm{c}}$ & 883 & $\mathrm{PO}_{4}{ }^{\mathrm{c}}$ & 579 & & \\
\hline \multicolumn{6}{|c|}{ Radiochemistry: Average Washed Solids } \\
\hline Analyte & $\begin{array}{c}\mu \mathrm{Ci} / \mathrm{g} \mathrm{dry} \\
\text { solids }^{\mathrm{f}}\end{array}$ & Analyte & $\begin{array}{c}\mu \mathrm{Ci} / \mathrm{g} \mathrm{dry} \\
\text { solids }\end{array}$ & Analyte & $\begin{array}{c}\mu \mathrm{Ci} / \mathrm{g} \mathrm{dry} \\
\text { solids }\end{array}$ \\
\hline $\mathrm{H}-3$ & $1.56 \mathrm{E}+2^{\mathrm{d}}$ & Cs-134 & $<7 \mathrm{E}-1$ & Am-241b & 203 \\
\hline C-14 & $2.36 \mathrm{E}-3$ & $\mathrm{Cs}-135,137^{\mathrm{e}}$ & 37 & Am-241 & 175 \\
\hline Co-60 & 7.40 & Cs-137 & 169 & $\mathrm{Cm}-242$ & $<0.06$ \\
\hline Sr-90 & $2.49 \mathrm{E}+4$ & Eu-152 & NM & $\mathrm{Cm}-243 / 244$ & 0.281 \\
\hline $\mathrm{Nb}-94$ & NM & Eu-154 & 72.8 & & \\
\hline Tc-99 & $2.64 \mathrm{E}-2$ & Eu-155 & 134 & & \\
\hline RuRh-106 & 11.8 & $\mathrm{~Np}-237^{\mathrm{e}}$ & $1.23 \mathrm{E}-1$ & & \\
\hline Sb-125 & 40.3 & $\mathrm{Pu}-236$ & $<0.3$ & & \\
\hline $\mathrm{Sn}-126^{\mathrm{e}}$ & $<2.7 \mathrm{E}-2$ & $\mathrm{Pu}-238$ & 1.57 & & \\
\hline $\mathrm{I}-129^{\mathrm{e}}$ & $<4.4 \mathrm{E}-8$ & $\mathrm{Pu}-239 / 240$ & 9.83 & & \\
\hline \multicolumn{6}{|c|}{$\begin{array}{l}\mathrm{a}=\text { Indicates the analyte value was taken from the } \mathrm{Na}_{2} \mathrm{O}_{2} \text { fusion preparation. } \\
\mathrm{b}=\text { Indicates the analyte value was taken from the } \mathrm{KOH} \text { fusion preparation. } \\
\mathrm{c}=\text { The analytical values for these anions have been reduced by multiplying by the factor } 624 / 954=0.654 \text { because } \\
\quad \text { during the compositing approximately } 330 \mathrm{~g} \text { of non-anion containing solids slurry was incorporated into the } \\
\quad \text { composite. } \\
\mathrm{d}=\text { tritium is measured in } \mu \mathrm{Ci} / \mathrm{g} \text { wet sample } \\
\mathrm{e}=\mathrm{ICP}-\mathrm{MS} \text { value } \\
\left.\mathrm{f}=\mu \mathrm{Ci} / \mathrm{g} \text { dry solids, analytical data are on a dry weight basis (dried at } 105^{\circ} \mathrm{C}\right) . \\
\mathrm{NM}=\text { not measured }\end{array}$} \\
\hline \multicolumn{6}{|c|}{$\begin{array}{l}\text { NOTE: Pretreated slurry properties: Composite Slurry Mass }=954.57 \mathrm{~g} \text {; solids } \mathrm{wt} \%=9.535 \% \text {; dry solids mass }= \\
91.02 \mathrm{~g} \text { that is }(76.51 \mathrm{wt} \% \text { oxide) is equivalent to } 69.64 \mathrm{~g} \text { oxide. }\end{array}$} \\
\hline
\end{tabular}


Table 3.3. Secondary Waste Compositions - Cs Ion Exchange Eluants

\begin{tabular}{|c|c|c|c|c|c|c|c|c|}
\hline \multicolumn{9}{|c|}{$\begin{array}{l}\text { Cs eluants: } 5 \text { bottles: 1) AW-101 Cs Eluant } 1 \text { composite, 2) AN-107 Cs Eluant } 2 \text { COMP, 3) AN-107 Cs } \\
\text { eluant } 1 \text { comp, 4) Cs IX Eluant column 2, run 1, 6/15/99, and 5) A1R Cs E composite. }\end{array}$} \\
\hline & \multicolumn{3}{|c|}{ Average } & \multirow{2}{*}{$\begin{array}{c}1 \\
\mu \mathrm{g} / \mathrm{mL}\end{array}$} & \multirow{2}{*}{$\begin{array}{c}2 \\
\mathrm{gg} / \mathrm{mL}\end{array}$} & \multirow{2}{*}{$\begin{array}{c}3 \\
\mu \mathrm{g} / \mathrm{mL}\end{array}$} & \multirow{2}{*}{$\begin{array}{c}4 \\
\mu \mathrm{g} / \mathrm{mL}\end{array}$} & \multirow{2}{*}{$\begin{array}{c}5 \\
\mu \mathrm{g} / \mathrm{mL}\end{array}$} \\
\hline element & $\mu \mathrm{g} / \mathrm{mL}$ & $O x: g / m L$ & Ave. Comp. wt\% oxide & & & & & \\
\hline$\overline{\mathrm{Al}}$ & 28.798 & $\begin{array}{l}54.410 \\
\end{array}$ & $\begin{array}{ll}2.35 \\
\end{array}$ & 14141 & 1.3 & 6.1 & 3.6 & 4.6 \\
\hline $\mathrm{B}$ & 21.163 & 68.150 & 2.94 & 72.6 & 7.78 & 7.42 & 12.5 & 13.8 \\
\hline $\mathrm{Ba}$ & 0.118 & 0.128 & 0.01 & 0.42 & 0.23 & -- & -- & -- \\
\hline $\mathrm{Ca}$ & 2.788 & 3.898 & 0.17 & 2.1 & 3.3 & 2.9 & -- & 5.3 \\
\hline $\mathrm{Cd}$ & 0.374 & 0.427 & 0.02 & 0.31 & 0.34 & 0.23 & 0.5 & 0.59 \\
\hline $\mathrm{Ce}$ & 0.000 & 0.000 & 0.00 & -- & -- & -- & -- & -- \\
\hline Co & 0.000 & 0.061 & 0.00 & & -- & -- & -- & -- \\
\hline $\mathrm{Cr}$ & 4.512 & 6.599 & 0.28 & 3.48 & 6.54 & 3.25 & 5.25 & 5.29 \\
\hline $\mathrm{Cs}^{\mathrm{a}}$ & 27.4 & 29.070 & 1.25 & 75.2 & 11.3 & 6.63 & 22.2 & 33.4 \\
\hline $\mathrm{Cu}$ & 24.177 & 30.268 & 1.31 & 50.6 & 12.7 & 7.13 & 20.3 & 39.3 \\
\hline $\mathrm{Fe}$ & 9.291 & 13.286 & 0.57 & 12.3 & 12.8 & 8.09 & 7.23 & 7.63 \\
\hline $\mathrm{K}$ & 159.862 & 192.572 & 8.30 & 382 & 190 & 210 & -- & -- \\
\hline $\mathrm{La}$ & 0.000 & 0.000 & 0.00 & -- & -- & -- & -- & -- \\
\hline $\mathrm{Mg}$ & 0.000 & 0.000 & 0.00 & & -- & -- & -- & -- \\
\hline $\mathrm{Mn}$ & 0.118 & 0.156 & 0.01 & 0.67 & -- & -- & -- & -- \\
\hline Mo & 0.000 & 0.087 & 0.00 & -- & -- & -- & -- & -- \\
\hline $\mathrm{Na}$ & 1308.463 & 1763.772 & 76.06 & 2230 & 698 & 1150 & 920 & 1630 \\
\hline $\mathrm{Nd}$ & 0.000 & 0.000 & 0.00 & -- & -- & -- & -- & -- \\
\hline $\mathrm{Ni}$ & 21.103 & 26.858 & 1.16 & 5.89 & 2.8 & 5.69 & 67.5 & 36.7 \\
\hline $\mathrm{P}$ & 0.522 & 1.196 & 0.05 & 2.9 & -- & -- & & \\
\hline $\mathrm{Pb}$ & 10.936 & 11.779 & 0.51 & 15.6 & 12.8 & 9.9 & 7.7 & 10 \\
\hline $\mathrm{Pd}$ & 0.000 & 0.000 & 0.00 & -- & -- & -- & -- & -- \\
\hline $\mathrm{Si}$ & 22.138 & 47.366 & 2.04 & 51.4 & 6.5 & 6.3 & 15 & 39 \\
\hline $\mathrm{Sn}$ & 0.000 & 0.000 & 0.00 & -- & -- & -- & -- & -- \\
\hline $\mathrm{Sr}$ & 0.227 & 0.272 & 0.01 & -- & -- & -- & 0.84 & 0.49 \\
\hline Th & 0.000 & 0.000 & 0.00 & -- & -- & -- & -- & -- \\
\hline $\mathrm{Ti}$ & 0.000 & 0.000 & 0.00 & -- & -- & -- & -- & -- \\
\hline $\mathrm{U}$ & 55.783 & 63.279 & 2.73 & 48 & -- & -- & 87 & 170 \\
\hline $\mathrm{Zn}$ & 4.394 & 5.467 & 0.24 & 12 & 6.2 & -- & 0.84 & 5.4 \\
\hline $\mathrm{Zr}$ & 0.000 & 0.000 & 0.00 & -- & -- & -- & -- & -- \\
\hline TOC & & & & 119 & $<18$ & $<18$ & 151 & 196 \\
\hline TIC & & & & & & & & \\
\hline $\mathrm{Br}$ & & & & $<500$ & $<100$ & $<100$ & $<100$ & $<100$ \\
\hline $\mathrm{Cl}$ & & & & $<500$ & $<100$ & $<100$ & $<100$ & $<100$ \\
\hline $\mathrm{F}$ & & & & $<500$ & $<100$ & $<100$ & $<100$ & $<100$ \\
\hline $\mathrm{NO}_{2}$ & & & & $<1000$ & $<200$ & $<200$ & $<200$ & $<200$ \\
\hline $\mathrm{NO}_{3}$ & & & & 33000 & 31500 & 26500 & 24500 & 26300 \\
\hline $\mathrm{PO}_{4}$ & & & & {$[2.9]$} & $<200$ & $<200$ & $<200$ & $<200$ \\
\hline $\mathrm{SO}_{4}$ & & & & $<1000$ & $<200$ & $<200$ & $<200$ & $<200$ \\
\hline oxalate & & & & $<1000$ & $<200$ & $<200$ & $<200$ & $<200$ \\
\hline & & & & & & & & \\
\hline $\begin{array}{l}\text { Volume } \\
(\mathrm{mL})\end{array}$ & & & & 194.877 & 181.970 & 304.926 & 173.179 & 211.545 \\
\hline Mass (g) & & & & 197.800 & 184.700 & 309.500 & 175.777 & 214.719 \\
\hline $\begin{array}{l}\text { Density } \\
(\mathrm{g} / \mathrm{mL})\end{array}$ & & & & 1.015 & 1.015 & 1.015 & 1.015 & 1.015 \\
\hline
\end{tabular}


Table 3.4. Secondary Waste Compositions - Tc Eluant Composite and Sr/TRU Precipitate

\begin{tabular}{|c|c|c|c|}
\hline Element & $\begin{array}{l}\text { Te Eluant composite, column } 1 . \\
\text { AW-101 Feed, High Nitrate }\end{array}$ & $\begin{array}{l}\text { Te Eluant composite, column1. } \\
\text { AN-107 Feed, Low Nitrate }\end{array}$ & $\begin{array}{l}\text { AN-107 DF large scale Sr/TRU } \\
\text { precipitation with real waste - } \\
\text { Overall Average }\end{array}$ \\
\hline & $\mu \mathrm{g} / \mathrm{g}$ & $\mu \mathrm{g} / \mathrm{g}$ & $\mu \mathrm{g} / \mathrm{g}$ \\
\hline $\mathrm{Ag}$ & -- & -- & 105 \\
\hline $\mathrm{Al}$ & 7.46 & 0.46 & 7915 \\
\hline B & 8.41 & 10.50 & -- \\
\hline $\mathrm{Ba}$ & 0.03 & 0.10 & 358 \\
\hline $\mathrm{Ca}$ & -- & 1.90 & 5413 \\
\hline $\mathrm{Cd}$ & -- & -- & 32.5 \\
\hline $\mathrm{Ce}$ & -- & -- & 1013 \\
\hline Co & -- & -- & -- \\
\hline $\mathrm{Cr}$ & 1.51 & -- & 3277 \\
\hline $\mathrm{Cu}$ & -- & -- & 53.3 \\
\hline $\mathrm{Fe}$ & 10.7 & -- & 47133 \\
\hline $\mathrm{K}$ & 23.8 & -- & -- \\
\hline $\mathrm{La}$ & -- & -- & 723 \\
\hline $\mathrm{Mg}$ & 0.13 & -- & 195 \\
\hline $\mathrm{Mn}$ & 0.15 & -- & 130000 \\
\hline Mo & -- & -- & 31 \\
\hline $\mathrm{Na}$ & 108 & 105 & 75567 \\
\hline $\mathrm{Nd}$ & -- & -- & 2117 \\
\hline $\mathrm{Ni}$ & 1.34 & 0.18 & 134 \\
\hline $\mathrm{P}$ & 0.24 & -- & 749 \\
\hline $\mathrm{Pb}$ & -- & -- & 4770 \\
\hline $\mathrm{Pd}$ & -- & -- & 987 \\
\hline $\mathrm{Si}$ & 16.7 & 22.50 & 4322 \\
\hline $\mathrm{Sn}$ & -- & -- & -- \\
\hline $\mathrm{Sr}$ & -- & -- & 272500 \\
\hline $\mathrm{Th}$ & -- & -- & 1150 \\
\hline $\mathrm{Ti}$ & -- & -- & 41.8 \\
\hline $\mathrm{U}$ & -- & -- & 1780 \\
\hline $\mathrm{Zn}$ & 0.12 & -- & 307 \\
\hline $\mathrm{Zr}$ & -- & -- & 1958 \\
\hline TOC & 42.7 & $<18$ & \\
\hline TIC & & not present in acidic samples & \\
\hline $\mathrm{Br}$ & & $<0.3$ & \\
\hline $\mathrm{Cl}$ & & 15 & \\
\hline $\mathrm{F}$ & & $<0.3$ & \\
\hline $\mathrm{NO}_{2}$ & & 8 & \\
\hline $\mathrm{NO}_{3}$ & 30399 & 19 & \\
\hline $\mathrm{PO}_{4}$ & & $<0.5$ & \\
\hline $\mathrm{SO}_{4}$ & & 1 & \\
\hline oxalate & & $<0.5$ & \\
\hline $\begin{array}{l}\text { Volume } \\
(\mathrm{mL})\end{array}$ & 340.8 & 194.5 & \\
\hline Mass $(\mathrm{g})$ & 343.0 & 194.5 & 29.09 \\
\hline $\begin{array}{l}\text { Density } \\
(\mathrm{g} / \mathrm{mL})\end{array}$ & 1.0066 & 1 & \\
\hline
\end{tabular}


Table 3.5. C-104 and AZ-102 Mineral and Chemical Glass Former Additives

\begin{tabular}{|c|c|c|c|c|c|}
\hline $\begin{array}{l}\text { Oxide } \\
\text { Needed }\end{array}$ & Source & $\begin{array}{c}\text { Company } \\
\text { Address \& telephone } \\
\end{array}$ & $\begin{array}{c}\text { Grade } \\
\text { Identification } \\
\end{array}$ & Wt\% of the oxide & $\begin{array}{c}\text { Principle other } \\
\text { oxides present }\end{array}$ \\
\hline $\mathrm{Al}_{2} \mathrm{O}_{3}{ }^{\mathrm{a}}$ & $\begin{array}{l}\text { Raw Kyanite } \\
\mathrm{Al}_{2} \mathrm{SiO}_{5}\end{array}$ & $\begin{array}{l}\text { Kyanite Mining Corp. } \\
\text { Dillwyn VA } 23936 \\
\text { (804) 983-2043 }\end{array}$ & $\begin{array}{l}\text { Raw Kyanite } \\
325 \text { MESH }\end{array}$ & $\overline{54 \%} \mathrm{Al}_{2} \mathrm{O}_{3}$ & $\begin{array}{l}43.7 \% \mathrm{SiO}_{2} \\
0.4 \% \mathrm{Fe}_{2} \mathrm{O}_{3}\end{array}$ \\
\hline $\mathrm{B}_{2} \mathrm{O}_{3}$ & $\mathrm{Na}_{2} \mathrm{~B}_{4} \mathrm{O}_{7} \cdot 10 \mathrm{H}_{2} \mathrm{O}$ & $\begin{array}{l}\text { NOAH Tech. Corp } \\
1 \text { NOAH Park } \\
\text { San Antonio, TX } 78249 \\
\text { (210) 691-2000 }\end{array}$ & $\mathrm{C} 2272$ & $\begin{array}{l}\text { Theory: } 36.51 \% \\
\mathrm{~B}_{2} \mathrm{O}_{3} \\
\text { Found: } 38.75 \%\end{array}$ & $\begin{array}{l}\mathrm{Na}_{2} \mathrm{O} 16.25 \% \\
\text { (Found } 16.6 \% \text { ) } \\
\\
\mathrm{CaO}<0.01 \%\end{array}$ \\
\hline $\mathrm{B}_{2} \mathrm{O}_{3}{ }^{\mathrm{a}, \mathrm{b}}$ & $\begin{array}{l}\text { Orthoboric Acid } \\
\mathrm{H}_{3} \mathrm{BO}_{3}\end{array}$ & $\begin{array}{l}\text { US Borax Inc. } \\
\text { 26877 Tourney Road } \\
\text { Valencia, CA 91355 } \\
\text { (660) 287-5400 }\end{array}$ & $\begin{array}{l}\text { Technical } \\
\text { Granular }\end{array}$ & $56.3 \% \mathrm{~B}_{2} \mathrm{O}_{3}$ & $\begin{array}{l}\text { None above } \\
0.01 \mathrm{wt} \%\end{array}$ \\
\hline $\mathrm{CaO}^{\mathrm{a}}$ & $\begin{array}{l}\text { Wollanstonite } \\
\mathrm{CaSiO}_{3}\end{array}$ & $\begin{array}{l}\text { NYCO Minerals } \\
124 \text { Mountain View Dr. } \\
\text { Willsboro, NY } 12996 \\
\text { (403) } 260-9883\end{array}$ & $\begin{array}{l}\text { Powder untreated } \\
\text { NYAD® } 325\end{array}$ & $47.5 \% \mathrm{CaO}$ & $\begin{array}{l}51.0 \% \mathrm{SiO}_{2} \\
0.4 \% \mathrm{Fe}_{2} \mathrm{O}_{3}\end{array}$ \\
\hline $\mathrm{Fe}_{2} \mathrm{O}_{3}{ }^{\mathrm{a}}$ & $\begin{array}{l}\text { Red Iron Oxide } \\
\text { Pigment } \\
\mathrm{Fe}_{2} \mathrm{O}_{3}\end{array}$ & $\begin{array}{l}\text { The Prince Man. Co. } \\
1 \text { Prince Plaza } \\
\text { Quincy, IL } 62306 \\
\text { (217) 222-8854 }\end{array}$ & $\begin{array}{l}\text { Red Iron Oxide } \\
5001\end{array}$ & $97 \% \mathrm{Fe}_{2} \mathrm{O}_{3}$ & $\begin{array}{l}1.50 \% \mathrm{Al}_{2} \mathrm{O}_{3} \\
1.35 \% \mathrm{SiO}_{2}\end{array}$ \\
\hline $\mathrm{Li}_{2} \mathrm{O}$ & $\mathrm{LiOH} \cdot \mathrm{H}_{2} \mathrm{O}$ & $\begin{array}{l}\text { NOAH Tech. Corp } \\
\text { (see above) }\end{array}$ & 15627 & $\begin{array}{l}\text { Theory: } 35.61 \% \\
\text { Found: } 33.78 \%\end{array}$ & $\begin{array}{l}\mathrm{Ca} / \mathrm{Fe} / \mathrm{K} \\
<10 \mathrm{ppm} \text { each }\end{array}$ \\
\hline $\mathrm{Li}_{2} \mathrm{O}$ & $\mathrm{LiOH}$ & $\begin{array}{l}\text { NOAH Tech. Corp } \\
\text { (see above) }\end{array}$ & & Theory: $62.38 \%$ & \\
\hline $\mathrm{Li}_{2} \mathrm{O}^{\mathrm{a}, \mathrm{b}}$ & $\mathrm{Li}_{2} \mathrm{CO}_{3}$ & $\begin{array}{l}\text { NOAH Tech. Corp } \\
\text { (see above) }\end{array}$ & 90815 & Theory: $40.43 \%$ & \\
\hline $\mathrm{MgO}^{\mathrm{a}}$ & 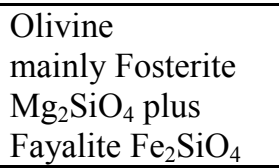 & $\begin{array}{l}\text { UNIMIN Corporation } \\
258 \text { Elm Street } \\
\text { New Canaan, CT } 06840 \\
\text { (203) 966-8880 }\end{array}$ & $\begin{array}{l}\text { Olivine } \\
\text { Grade } 180 \\
\text { Green Mountain, } \\
\text { NC } \\
\end{array}$ & $48.01 \mathrm{wt} \% \mathrm{MgO}$ & $\begin{array}{l}42.52 \mathrm{wt} \% \mathrm{SiO}_{2} \\
7.68 \mathrm{wt} \% \mathrm{Fe}_{2} \mathrm{O}_{3}\end{array}$ \\
\hline $\mathrm{SiO}_{2}^{\mathrm{a}, \mathrm{b}}$ & $\begin{array}{l}\text { Ground Silica } \\
\mathrm{Sand} \\
\mathrm{SiO}_{2}\end{array}$ & $\begin{array}{l}\text { US Silica Company } \\
\text { P.O. Box 187 } \\
\text { Berkeley Springs } \\
\text { WV, 25411-0187 } \\
(800) 243-7500\end{array}$ & SIL-CO-SIL $® 75$ & $99.5 \% \mathrm{SiO}_{2}$ & $0.3 \% \mathrm{Al}_{2} \mathrm{O}_{3}$ \\
\hline $\mathrm{Na}_{2} \mathrm{O}^{\mathrm{b}}$ & $\begin{array}{l}\text { Sodium Carbonate } \\
\mathrm{Na}_{2} \mathrm{CO}_{3}\end{array}$ & Fisher Scientific Co. & Reagent Grade & $99.8 \% \mathrm{Na}_{2} \mathrm{CO}_{3}$ & $\begin{array}{l}0.2 \% \text { loss on } \\
\text { heating to } 285^{\circ} \mathrm{C}\end{array}$ \\
\hline & Sucrose $e^{a, b, c}$ & To Be Determined & & & \\
\hline $\mathrm{TiO}_{2}{ }^{\mathrm{a}}$ & $\begin{array}{l}\text { Rutile Ore } \\
\mathrm{TiO}_{2}\end{array}$ & $\begin{array}{l}\text { Chemalloy Company } \\
\text { P.O. Box } 350 \\
\text { Bryn Mawr, PA } 19010 \\
\text { (610) 527-3700 }\end{array}$ & $\begin{array}{l}\text { Premium Grade } \\
\text { Rutile Ore } \\
\text { Airfloated }\end{array}$ & $95.4 \% \mathrm{TiO}_{2}$ & $\begin{array}{l}0.91 \% \mathrm{SiO}_{2} \\
0.90 \% \mathrm{ZrO}_{2} \\
0.71 \% \mathrm{Fe}_{2} \mathrm{O}_{3} \\
0.41 \% \mathrm{~V}_{2} \mathrm{O}_{5} \\
\end{array}$ \\
\hline $\mathrm{ZrO}_{2}{ }^{\mathrm{a}}$ & $\begin{array}{l}\text { Zircon Sand } \\
\text { Zirconium silicate } \\
\mathrm{ZrSiO}_{4}\end{array}$ & $\begin{array}{l}\text { American Minerals Inc. } \\
901 \text { E. } 8^{\text {th }} \text { Ave., \#200 } \\
\text { King of Prussia, PA } 19406 \\
(610) 337-8030\end{array}$ & Flour 325 MESH & $66 \% \mathrm{ZrO}_{2}\left(+\mathrm{HfO}_{2}\right)$ & $34 \% \mathrm{SiO}_{2}$ \\
\hline $\mathrm{ZnO}^{\mathrm{a}, \mathrm{b}}$ & $\begin{array}{l}\text { Zinc Oxide } \\
\mathrm{ZnO}\end{array}$ & $\begin{array}{l}\text { Zinc Corp. of America } \\
300 \text { Frankfort Road } \\
\text { Monaca, PA } 15061 \\
\text { (724) 774-1020 }\end{array}$ & KADOX-920 & $99.8 \% \mathrm{ZnO}$ & $\begin{array}{l}\text { None above } \\
0.01 \mathrm{wt} \%\end{array}$ \\
\hline
\end{tabular}




\section{HLW Glass Melter Feed Batching}

The amount of pretreated HLW tank sludge samples along with the necessary flowsheet amounts of secondary wastes (radionuclide rich streams, i.e. Cs and Tc ion exchange eluants and Sr/TRU precipitates) to produce each of the HLW glass melter feeds are described below.

\section{$\underline{\text { C-104 Melter Feed Batching }}$}

Radioactive C-104 melter feed was made using HLW pretreated sludge solids, Cs ion exchange eluant, and both actual and simulated Sr/TRU precipitate solids. Table 3.6 provides the amounts and compositions of the required secondary waste additions of AN-107 Sr/TRU precipitate plus AN-107 simulant Sr/TRU precipitate solids and cesium ion exchange eluant to the initial C-104 pretreated sludge waste to batch to the correct target composition for 100 grams of glass. Tables 3.7 and 3.8 give the actual amounts of the various components to make 250 grams of glass.

Table 3.6. Waste Component Compositions and Batching Ratios to Make up the C-104 Melter Feed

\begin{tabular}{|c|c|c|c|c|c|}
\hline Oxide & $\begin{array}{l}\mathrm{C}-104 \\
\mathrm{Wt} \% \\
\end{array}$ & $\begin{array}{c}\text { Composited } \\
\text { Cs Eluant } \\
\text { Wt } \% \\
\end{array}$ & \begin{tabular}{|c} 
Composited \\
Actual- \\
Simulant Sr/TRU \\
Wt\% \\
\end{tabular} & $\begin{array}{c}\text { Glass former } \\
\text { Composition } \\
\text { Grams/ 100g Glass } \\
\end{array}$ & $\begin{array}{c}\text { Estimated Waste } \\
\text { Glass Batch Feed } \\
\text { Composition } \\
\text { Wt\% } \\
\end{array}$ \\
\hline $\mathrm{Ag}_{2} \mathrm{O}$ & 0.256 & 0 & 0.01 & \multirow{19}{*}{13.435} & 0.065 \\
\hline $\mathrm{Al}_{2} \mathrm{O}_{3}$ & 8.723 & 2.35 & 1.95 & & 2.358 \\
\hline $\mathrm{B}_{2} \mathrm{O}_{3}$ & 0.021 & 2.94 & 0.00 & & 9.008 \\
\hline $\mathrm{BaO}$ & 0.060 & 0.01 & 0.05 & & 0.019 \\
\hline $\mathrm{BeO}$ & 0.020 & 0.00 & 0.00 & & 0.005 \\
\hline $\mathrm{Bi}_{2} \mathrm{O}_{3}$ & 0.010 & 0.00 & 0.00 & & 0.003 \\
\hline $\mathrm{CaO}$ & 1.505 & 0.17 & 1.03 & & 0.457 \\
\hline $\mathrm{CdO}$ & 0.240 & 0.02 & 0.00 & & 0.061 \\
\hline $\mathrm{Ce}_{2} \mathrm{O}_{3}$ & 0.275 & 0.00 & 0.16 & & 0.081 \\
\hline $\mathrm{CoO}$ & 0.009 & 0.00 & 0.00 & & 0.002 \\
\hline $\mathrm{Cr}_{2} \mathrm{O}_{3}$ & 0.359 & 0.28 & 0.63 & & 0.138 \\
\hline $\mathrm{Cs}_{2} \mathrm{O}$ & 0.027 & 1.25 & 0.00 & & 0.013 \\
\hline $\mathrm{CuO}$ & 0.073 & 1.31 & 0.01 & & 0.026 \\
\hline $\mathrm{Dy}_{2} \mathrm{O}_{3}$ & 0.011 & 0.00 & 0.00 & & 0.003 \\
\hline $\mathrm{Eu}_{2} \mathrm{O}_{3}$ & 0.005 & 0.00 & 0.00 & & 0.001 \\
\hline $\mathrm{Fe}_{2} \mathrm{O}_{3}$ & 16.013 & 0.57 & 9.11 & & 4.717 \\
\hline $\mathrm{HgO}$ & 0.004 & 0.00 & & & 0.001 \\
\hline $\mathrm{K}_{2} \mathrm{O}$ & 0.076 & 8.30 & 0.00 & & 0.060 \\
\hline $\mathrm{La}_{2} \mathrm{O}_{3}$ & 0.043 & 0.00 & 0.11 & & 0.019 \\
\hline $\mathrm{Li}_{2} \mathrm{O}$ & 0.129 & 0.00 & 0.00 & \multirow[t]{4}{*}{7.432} & 5.005 \\
\hline $\mathrm{MgO}$ & 0.222 & 0.00 & 0.04 & & 0.059 \\
\hline $\mathrm{MnO}$ & 3.195 & 0.01 & 23.68 & & 2.548 \\
\hline $\mathrm{MoO}_{3}$ & 0.006 & 0.00 & 0.01 & & 0.002 \\
\hline $\mathrm{Na}_{2} \mathrm{O}$ & 9.925 & 76.06 & 13.73 & \multirow[t]{4}{*}{6.989} & 8.565 \\
\hline $\mathrm{Nd}_{2} \mathrm{O}_{3}$ & 0.082 & 0.00 & 0.32 & & 0.044 \\
\hline $\mathrm{NiO}$ & 0.907 & 1.16 & 0.02 & & 0.236 \\
\hline $\mathrm{P}_{2} \mathrm{O}_{5}$ & 1.237 & 0.05 & 0.24 & & 0.330 \\
\hline
\end{tabular}




\begin{tabular}{|c|c|c|c|c|c|}
\hline Oxide & $\begin{array}{l}\text { C-104 } \\
\text { Wt\% } \\
\end{array}$ & $\begin{array}{c}\text { Composited } \\
\text { Cs Eluant } \\
\text { Wt\% } \\
\end{array}$ & \begin{tabular}{|c} 
Composited \\
Actual- \\
Simulant Sr/TRU \\
Wt\% \\
\end{tabular} & $\begin{array}{c}\text { Glass former } \\
\text { Composition } \\
\text { Grams/ 100g Glass } \\
\end{array}$ & $\begin{array}{c}\text { Estimated Waste } \\
\text { Glass Batch Feed } \\
\text { Composition } \\
\mathbf{W t} \% \\
\end{array}$ \\
\hline $\mathrm{PbO}$ & 0.400 & 0.51 & 0.68 & & 0.154 \\
\hline $\mathrm{PdO}$ & 0.000 & 0.00 & 0.15 & & 0.011 \\
\hline $\operatorname{Pr}_{6} \mathrm{O}_{11}$ & 0.019 & 0.00 & & & 0.005 \\
\hline $\mathrm{PtO}_{2}$ & 0.000 & 0.00 & & & 0.000 \\
\hline $\mathrm{Rh}_{2} \mathrm{O}_{3}$ & 0.128 & 0.00 & 0.00 & & 0.032 \\
\hline $\mathrm{RuO}_{2}$ & 0.065 & 0.00 & 0.00 & & 0.016 \\
\hline $\mathrm{SeO}_{2}$ & 0.014 & 0.00 & 0.00 & & 0.004 \\
\hline $\mathrm{SiO}_{2}$ & 5.906 & 2.04 & 1.20 & 69.172 & 47.866 \\
\hline $\mathrm{SnO}_{2}$ & 0.272 & 0.00 & 0.00 & & 0.069 \\
\hline $\mathrm{SrO}$ & 0.028 & 0.01 & 46.01 & & 3.389 \\
\hline $\mathrm{Ta}_{2} \mathrm{O}_{5}$ & 0.000 & 0.00 & 0.00 & & 0.000 \\
\hline $\mathrm{ThO}_{2}$ & 16.184 & 0.00 & 0.17 & & 4.101 \\
\hline $\mathrm{TiO}_{2}$ & 0.063 & 0.00 & 0.01 & & 0.017 \\
\hline $\mathrm{UO}_{2}$ & 14.260 & 2.73 & 0.26 & & 3.635 \\
\hline $\mathrm{V}_{2} \mathrm{O}_{5}$ & 0.015 & 0.00 & 0.00 & & 0.004 \\
\hline $\mathrm{Y}_{2} \mathrm{O}_{3}$ & 0.012 & 0.00 & 0.00 & & 0.003 \\
\hline $\mathrm{ZnO}$ & 0.128 & 0.24 & 0.05 & 2.936 & 2.001 \\
\hline $\mathrm{ZrO}_{2}$ & 19.075 & 0.00 & 0.34 & & 4.843 \\
\hline waste composition & 76.298 & 1.486 & 22.216 & & \\
\hline waste to glass formers & $25.255 \%$ & $0.492 \%$ & $7.353 \%$ & $66.900 \%$ & $100 \%$ \\
\hline
\end{tabular}

Table 3.7. C-104 Pretreated Sludge Waste and Secondary Waste Additions to Produce C-104 Melter Feed for 250g of Glass

\begin{tabular}{|c|c|}
\hline Waste Type & Added to C-104* Melter Feed Batch \\
\hline \hline C-104 Pretreated Sludge Waste & $400.5 \mathrm{~g}$ \\
\hline Sr/TRU precipitates including simulant & $25.547 \mathrm{~g}$ dried solids \\
\hline Tc Eluant & NOT ADDED \\
\hline Cs Eluant - Average & 529.0 g of Cs Eluant \\
\hline *Based on making 250 grams of glass \\
\hline
\end{tabular}

Table 3.8. Grams of Mineral Additives Added to the Waste Slurry to Make 250g of C-104 Glass

\begin{tabular}{|c|c|c|}
\hline Oxide & Additive Compound & Added to C-104 Melter Feed Batch \\
\hline \hline $\mathrm{B}_{2} \mathrm{O}_{3}$ & $\mathrm{Na}_{2} \mathrm{~B}_{4} \mathrm{O}_{7} \cdot 10 \mathrm{H}_{2} \mathrm{O}$ & 58.0 \\
\hline $\mathrm{Li}_{2} \mathrm{O}$ & $\mathrm{LiOH} \cdot \mathrm{H}_{2} \mathrm{O}$ & 36.8 \\
\hline $\mathrm{Na}_{2} \mathrm{O}$ & $\mathrm{Na}_{2} \mathrm{CO}_{3}$ & 3.525 \\
\hline $\mathrm{SiO}_{2}$ & $\mathrm{SiO}_{2}(\mathrm{Sil}-\mathrm{co}-\mathrm{Sil} 75)$ & 116.28 \\
\hline $\mathrm{ZnO}$ & $\mathrm{ZnO}(\mathrm{K}-920)$ & 4.93 \\
\hline
\end{tabular}




\section{AZ-102 Melt 1 and AZ-102 Melt 2 Melter Feed Batching}

It was necessary to complete both the physical and rheological properties tests and the vitrification and product testing scopes of work related to the pretreated AZ-102 tank sludge waste simultaneously; therefore, the pretreated AZ-102 waste was split into nearly equal amounts to make two AZ-102 glass melts. The first melt, AZ-102 Melt 1, did not have sugar added to the melter feed, i.e. did not simulate the actual RPP-WTP HLW flowsheet, as it will not affect the crucible melt final glass product composition or properties being tested (PCT, crystalline and noncrystalline phase determination, chemical and radiochemical composition analysis, and regulatory analysis except for TCLP and SVOA). The second melt, AZ-102 Melt 2, was generated from the physical and rheological properties portion of the AZ-102 pretreated waste split that contained sugar in flowsheet amounts as sugar addition was necessary to accurately measure the melter feed flowsheet "Physical and Rheological Properties." Both AZ-102 Melt 1 and Melt 2 melter feed batching requirements are explained separately below.

\section{AZ-102 Melt 1 Melter Feed Batching}

Radioactive AZ-102 Melt 1 melter feed was made using HLW pretreated sludge solids, Cs ion exchange eluant, Tc ion exchange eluant, and simulated Sr/TRU precipitate solids. Table 3.9 provides the amounts and compositions of the required secondary waste additions of AN-107 simulant Sr/TRU precipitate solids and cesium and technetium ion exchange eluants to the initial AZ-102 pretreated sludge waste to batch to the correct target composition for 100 grams of glass. Tables 3.10 and 3.11 give the actual amounts of the various components to make 118.92 grams of glass.

Table 3.9. Recipe for HLW AZ-102 Melt 1 Glass Composition from VSL, Using Pretreated Waste

\begin{tabular}{|c|c|c|c|c|c|c|c|}
\hline & AZ102 & AN107 & Cs Eluant & $\begin{array}{c}\text { Tc Eluant, AW-101 } \\
\&\end{array}$ & Blended & Additives & Glass \\
\hline \multirow[t]{2}{*}{ Oxide } & Solid & $\begin{array}{l}\text { (Sr/TRU) } \\
\text { simulated }\end{array}$ & Composite & AN-107 Composite & Waste & & Composition \\
\hline & $(w t \%)$ & $(w t \%)$ & $(w t \%)$ & $(\mathbf{w t} \%)$ & $(w t \%)$ & (wt\% of Glass) & $(w t \%)$ \\
\hline $\mathrm{Ag}_{2} \mathrm{O}$ & 0.060 & 0.00 & 0.00 & & 0.055 & & 0.018 \\
\hline $\mathrm{Al}_{2} \mathrm{O}_{3}$ & 25.070 & 0.00 & 2.35 & 3.52 & 23.013 & & 7.684 \\
\hline $\mathrm{B}_{2} \mathrm{O}_{3}$ & 0.030 & 0.00 & 2.94 & 11.34 & 0.060 & 3.96 & 3.980 \\
\hline $\mathrm{BaO}$ & 0.120 & 0.03 & 0.01 & 0.02 & 0.112 & & 0.037 \\
\hline $\mathrm{BeO}$ & 0.010 & 0.00 & 0.00 & & 0.009 & & 0.003 \\
\hline $\mathrm{CaO}$ & 1.509 & 0.61 & 0.17 & 0.38 & 1.430 & & 0.478 \\
\hline $\mathrm{CdO}$ & 4.558 & 0.00 & 0.02 & & 4.180 & & 1.396 \\
\hline $\mathrm{CeO}_{2}$ & 0.190 & 0.03 & 0.00 & & 0.176 & & 0.059 \\
\hline $\mathrm{Cl}$ & 0.160 & & 0.00 & 2.13 & 0.148 & & 0.049 \\
\hline $\mathrm{CoO}$ & 0.020 & 0.00 & 0.00 & & 0.018 & & 0.006 \\
\hline $\mathrm{Cr}_{2} \mathrm{O}_{3}$ & 0.290 & 0.11 & 0.28 & 0.53 & 0.277 & & 0.092 \\
\hline $\mathrm{Cs}_{2} \mathrm{O}$ & 0.020 & & 1.25 & & 0.030 & & 0.010 \\
\hline $\mathrm{CuO}$ & 0.080 & 0.01 & 1.31 & & 0.086 & & 0.029 \\
\hline $\mathrm{F}$ & 0.040 & 0.00 & 0.00 & & 0.037 & & 0.012 \\
\hline $\mathrm{Fe}_{2} \mathrm{O}_{3}$ & 39.254 & 4.94 & 0.57 & 3.69 & 36.367 & & 12.143 \\
\hline $\mathrm{K}_{2} \mathrm{O}$ & 0.000 & 0.00 & 8.30 & 6.92 & 0.079 & & 0.026 \\
\hline $\mathrm{La}_{2} \mathrm{O}_{3}$ & 0.980 & 0.01 & 0.00 & & 0.899 & & 0.300 \\
\hline $\mathrm{Li}_{2} \mathrm{O}$ & 0.000 & 0.00 & 0.00 & & 0.000 & 5.00 & 5.000 \\
\hline $\mathrm{MgO}$ & 0.390 & 0.00 & 0.00 & 0.05 & 0.358 & & 0.119 \\
\hline $\mathrm{MnO}$ & 0.850 & 27.02 & 0.01 & 0.05 & 2.762 & & 0.922 \\
\hline $\mathrm{MoO}_{3}$ & 0.000 & 0.00 & 0.00 & & 0.000 & & 0.000 \\
\hline
\end{tabular}




\begin{tabular}{|c|c|c|c|c|c|c|c|}
\hline & AZ102 & AN107 & Cs Eluant & $\begin{array}{c}\text { Tc Eluant, AW-101 } \\
\&\end{array}$ & Blended & Additives & Glass \\
\hline \multirow[t]{2}{*}{ Oxide } & Solid & $\begin{array}{l}\text { (Sr/TRU) } \\
\text { simulated }\end{array}$ & Composite & AN-107 Composite & Waste & & Composition \\
\hline & $(w t \%)$ & $(w t \%)$ & $(\mathbf{w t} \%)$ & $(w t \%)$ & $(w t \%)$ & (wt\% of Glass) & $(w t \%)$ \\
\hline $\mathrm{Na}_{2} \mathrm{O}$ & 8.397 & 6.77 & 76.06 & 55.27 & 8.916 & 10.29 & 13.267 \\
\hline $\mathrm{Nd}_{2} \mathrm{O}_{3}$ & 0.680 & 0.04 & 0.00 & & 0.627 & & 0.209 \\
\hline $\mathrm{NiO}$ & 2.489 & 0.02 & 1.16 & 0.44 & 2.295 & & 0.766 \\
\hline $\mathrm{P}_{2} \mathrm{O}_{5}$ & 1.499 & 0.21 & 0.05 & 0.13 & 1.391 & & 0.464 \\
\hline $\mathrm{PbO}$ & 0.290 & 0.16 & 0.51 & & 0.282 & & 0.094 \\
\hline $\mathrm{PdO}$ & 0.000 & 0.00 & 0.00 & & 0.000 & & 0.000 \\
\hline $\mathrm{SO}_{3}$ & 0.070 & 0.00 & 0.00 & & 0.064 & & 0.021 \\
\hline $\mathrm{SiO}_{2}$ & 2.049 & 0.00 & 2.04 & 15.47 & 1.905 & 47.36 & 47.996 \\
\hline $\mathrm{SnO}_{2}$ & 0.540 & 0.00 & 0.00 & & 0.495 & & 0.165 \\
\hline $\mathrm{SrO}$ & 0.070 & 60.00 & 0.01 & & 4.466 & & 1.491 \\
\hline $\mathrm{ThO}_{2}$ & 0.000 & 0.00 & 0.00 & & 0.000 & & 0.000 \\
\hline $\mathrm{TiO}_{2}$ & 0.030 & 0.00 & 0.00 & & 0.028 & & 0.009 \\
\hline $\mathrm{UO}_{2}$ & 5.278 & 0.00 & 2.73 & & 4.865 & & 1.624 \\
\hline $\mathrm{Y}_{2} \mathrm{O}_{3}$ & 0.050 & 0.00 & 0.00 & & 0.046 & & 0.015 \\
\hline $\mathrm{ZnO}$ & 0.130 & 0.04 & 0.24 & 0.04 & 0.124 & & 0.042 \\
\hline \multirow[t]{5}{*}{$\mathrm{ZrO}_{2}$} & 4.798 & 0.00 & 0.00 & & 4.400 & & 1.469 \\
\hline & $100.00 \%$ & $100.00 \%$ & $100.00 \%$ & $100.00 \%$ & $100.00 \%$ & $66.61 \%$ & $100.00 \%$ \\
\hline & & & & & & & \\
\hline & 250.00 & 20.000 & 2.479 & 0.1367 & & \multirow{2}{*}{$\begin{array}{c}\text { Total Waste } \\
\text { Loading } \\
\text { AZ102 Loading }\end{array}$} & $33.39 \%$ \\
\hline & & & & 272.616 & & & $30.62 \%$ \\
\hline
\end{tabular}

Table 3.10. AZ-102 Pretreated Waste and Secondary Waste Additions to Melter Feed

\begin{tabular}{|c|c|}
\hline \multicolumn{1}{|c|}{ Waste and Secondary Waste } & AZ-102 Melt 1 \\
\hline \hline AZ-102 Pretreated Sludge Waste & 499.02 g slurry \\
\hline AN-107 Simulant Sr/TRU Precipitate & $14.37 \mathrm{~g}$ wet solids \\
\hline Tc Eluant - low nitrate & $69.38 \mathrm{~g}$ eluant \\
\hline Tc Eluant - high nitrate & $11.37 \mathrm{~g}$ eluant \\
\hline Cs Eluant - Average & $157.29 \mathrm{~g}$ eluant \\
\hline $\begin{array}{l}\text { Note: } \\
\text { Densities of Tc eluants are assumed to be } 1.00 \mathrm{~g} / \mathrm{mL} . \\
\text { Density of composite Cs eluants is assumed to be } 1.015 \mathrm{~g} / \mathrm{mL} .\end{array}$ \\
\hline
\end{tabular}


Table 3.11. Grams of Mineral Additives added to the Waste Slurry to Make $118.92 \mathrm{~g}$ of Glass from the AZ-102 Melt 1 Melter Feed

\begin{tabular}{|c|c|c|}
\hline Oxides & Additives & AZ-102 Melt 1 \\
\hline $\mathrm{B}_{2} \mathrm{O}_{3}$ & $\mathrm{Na}_{2} \mathrm{~B}_{4} \mathrm{O}_{7} \cdot 10 \mathrm{H}_{2} \mathrm{O}$ & $12.15 \mathrm{~g}$ \\
\hline $\mathrm{Li}_{2} \mathrm{O}$ & $\mathrm{LiOH} \cdot \mathrm{H}_{2} \mathrm{O}$ & $17.60 \mathrm{~g}$ \\
\hline $\mathrm{Na}_{2} \mathrm{O}$ & $\mathrm{Na}_{2} \mathrm{SiO}_{3} \cdot 5 \mathrm{H}_{2} \mathrm{O}$ & $34.95 \mathrm{~g}$ \\
\hline $\mathrm{SiO}_{2}$ & $\mathrm{SiO}_{2}(\mathrm{Sil}-\mathrm{co}-\mathrm{Sil} 75)$ & $46.66 \mathrm{~g}$ \\
\hline \multicolumn{3}{|c|}{$\begin{array}{l}\text { Note: The correct amount of } \mathrm{B}_{2} \mathrm{O}_{3} \text { is determined by addition of } \mathrm{Na}_{2} \mathrm{~B}_{4} \mathrm{O}_{7} \cdot 10 \mathrm{H}_{2} \mathrm{O} \text { which adds } \mathrm{Na}_{2} \mathrm{O} \\
\text { as well; the correct amount of } \mathrm{Na}_{2} \mathrm{O} \text { is then calculated and by taking into account the amount of } \\
\mathrm{Na}_{2} \mathrm{O} \text { added from the borax, the difference is provided by } \mathrm{Na}_{2} \mathrm{SiO}_{3} \cdot 5 \mathrm{H}_{2} \mathrm{O} \text { which adds } \mathrm{SiO}_{2} \text { as well; } \\
\text { finally, the correct amount of } \mathrm{SiO}_{2} \text { is calculated and by taking into account the amount of } \mathrm{SiO}_{2} \\
\text { added from the sodium metasilicate pentahydrate, the difference is provided by } \mathrm{SiO}_{2} \text {. }\end{array}$} \\
\hline
\end{tabular}

\section{$\underline{\text { AZ-102 Melt } 2 \text { Melter Feed Batching }}$}

Radioactive AZ-102 Melt 2 melter feed was made using HLW pretreated sludge solids, Cs ion exchange eluant, Tc ion exchange eluant, actual Sr/TRU precipitate solids, and sugar. Table 3.12 provides the amounts and compositions of the required secondary waste additions of AN-107 Sr/TRU precipitate plus AN-107 simulant Sr/TRU precipitate solids and cesium and technetium ion exchange eluants to the initial AZ-102 pretreated sludge waste to batch to the correct target composition for 100 grams of glass. Tables 3.13 and 3.14 give the actual amounts of the various components to make 69.81 grams of glass. Note that not all of the melter feed was available for melting. One portion was under going long term aging studies and would not be available in time to allow follow-on analytical work to be completed in time to be reported.

As sugar was added to this melter feed to simulate the actual melter feed for physical and rheological testing purposes the following hazards assessment was completed to assess potential problems in the HLRF hot cell during radioactive glass preparation, and a simulant melt of the hazard analysis AZ-102 Melt 2 was performed before actual radioactive processing:

Nitrate and sugar in the HLW melter feeds will undergo an oxidation - reduction reaction as the melter feed dries out and heats up in a calcining or melting furnace. This reaction may be energetic enough to heat the dried feed to incandescence $\left(.600^{\circ} \mathrm{C}\right)$. The reaction in the presence of non-reactive feed particulate has been observed to propagate slowly through the dried feed. Overall these reactions do not constitute a hazard.

The Catholic University of America, RPP-WTP team member responsible for all melter feed and glass formulations, has formulated the HLW melter feed to have 12 carbons per 16 nitrates to reduce the nitrate present and hence foaming when the melter feed is melted. The overall reaction will be similar to the following:

$$
\mathrm{C}_{12} \mathrm{H}_{22} \mathrm{O}_{11}+16 \mathrm{NaNO}_{3} \leftrightarrow 8 \mathrm{Na}_{2} \mathrm{O}+16 \mathrm{NO}+12 \mathrm{CO}_{2}+11 \mathrm{H}_{2} \mathrm{O}
$$

which is conservatively high with respect to the amount of gas generated. In this reaction, one mole of sucrose produces as much as 39 moles of gas, e.g. in the HLW AZ-102 Melt 2 melter feed, 1.6 grams of sugar ( 0.0047 moles) will be added to the batch AZ-102 HLW pretreated waste which may result in the production of as much as 0.182 moles of gas (4.1 liters at standard temperature and pressure (STP)). 
The likelihood of over pressurization is nonexistent because the reaction will occur in a completely unconfined system, the reaction time will be on the order of tens of seconds, and some of the reaction gases may react with batch components such as $\mathrm{CO}_{2}$ to form carbonates or condense on cooler surfaces $\left(\mathrm{H}_{2} \mathrm{O}\right)$ which would lower the gas volume. These reactions will occur in a hot cell with a volume of about 15000 liters, which is significantly larger than 4.1 liters. In addition, the air volume in the cell turns over about once per minute (15000 liters/min. or 250 liters per second). It would take approximately sixty times the reactant loading reacting each second to equal the exhaust rate of this cell. Hence, if the amount of sucrose to be reacted with nitrate in the hot cell was about 80 grams, the entire amount would have to react in less than one second for the cell pressure differential to approach zero. Based on this evaluation, it was concluded that the high level melts could be made in the HLRF with no risk from the nitrate- sugar reaction.

Table 3.12. Waste Compositions and Batching Ratios for the AZ-102 Melt 2 Waste Glass

\begin{tabular}{|c|c|c|c|c|c|c|c|}
\hline Oxides & $\begin{array}{c}\text { AZ102 } \\
\text { Solid } \\
(\mathbf{w t} \%) \\
\end{array}$ & $\begin{array}{c}\text { AN-107 Sr/TRU } \\
\text { ppt Composite } \\
\text { (wt\%) }\end{array}$ & $\begin{array}{c}\text { Cs Eluant } \\
\text { Composite } \\
(\mathbf{w t} \%) \\
\end{array}$ & $\begin{array}{c}\text { Tc Eluant } \\
\text { Low Nitrate } \\
\text { (wt\%) } \\
\end{array}$ & $\begin{array}{c}\text { Blended } \\
\text { Waste } \\
(\mathbf{w t} \%) \\
\end{array}$ & $\begin{array}{c}\text { Additives } \\
\text { wt } \% \text { of } \\
\text { Waste Glass } \\
\end{array}$ & $\begin{array}{c}\text { AZ-102 Melt } 2 \text { VSL-2 } \\
\text { Melt2_27 } \\
\text { wt\% ox. } \\
\end{array}$ \\
\hline $\mathrm{Ag}_{2} \mathrm{O}$ & 0.060 & 0.016 & 0.00 & 0.00 & 0.06 & & 0.02 \\
\hline $\mathrm{Al}_{2} \mathrm{O}_{3}$ & 25.070 & 2.089 & 2.35 & 0.35 & 22.64 & & 7.69 \\
\hline $\mathrm{B}_{2} \mathrm{O}_{3}$ & 0.030 & 0.000 & 2.94 & 13.95 & 0.06 & 3.98 & 4.00 \\
\hline $\mathrm{BaO}$ & 0.120 & 0.056 & 0.01 & 0.05 & 0.11 & & 0.04 \\
\hline $\mathrm{BeO}$ & 0.010 & & 0.00 & 0.00 & 0.01 & & 0.00 \\
\hline $\mathrm{CaO}$ & 1.509 & 1.058 & 0.17 & 1.10 & 1.45 & & 0.49 \\
\hline $\mathrm{CdO}$ & 4.558 & 0.005 & 0.02 & 0.00 & 4.08 & & 1.38 \\
\hline $\mathrm{CeO}_{2}$ & 0.190 & 0.166 & 0.00 & 0.00 & 0.19 & & 0.06 \\
\hline $\mathrm{Cl}$ & 0.160 & & 0.00 & 6.19 & 0.15 & & 0.05 \\
\hline $\mathrm{CoO}$ & 0.020 & 0.000 & & 0.00 & 0.02 & & 0.01 \\
\hline $\mathrm{Cr}_{2} \mathrm{O}_{3}$ & 0.290 & 0.669 & 0.28 & 0.00 & 0.33 & & 0.11 \\
\hline $\mathrm{Cs}_{2} \mathrm{O}$ & 0.020 & & 1.25 & & 0.03 & & 0.01 \\
\hline $\mathrm{CuO}$ & 0.080 & 0.009 & 1.31 & 0.00 & 0.08 & & 0.03 \\
\hline $\mathrm{F}$ & 0.040 & & & 0.00 & 0.04 & & 0.01 \\
\hline $\mathrm{Fe}_{2} \mathrm{O}_{3}$ & 39.254 & 9.412 & 0.57 & 0.00 & 36.01 & & 12.23 \\
\hline $\mathrm{K}_{2} \mathrm{O}$ & 0.000 & 0.000 & 8.30 & 0.00 & 0.07 & & 0.03 \\
\hline $\mathrm{La}_{2} \mathrm{O}_{3}$ & 0.980 & 0.118 & 0.00 & 0.00 & 0.89 & & 0.30 \\
\hline $\mathrm{Li}_{2} \mathrm{O}$ & 0.000 & & 0.00 & 0.00 & 0.00 & 5 & 5.00 \\
\hline $\mathrm{MgO}$ & 0.390 & 0.045 & 0.00 & 0.00 & 0.35 & & 0.12 \\
\hline $\mathrm{MnO}$ & 0.850 & 23.443 & 0.01 & 0.00 & 3.02 & & 1.03 \\
\hline $\mathrm{MoO}_{3}$ & 0.000 & 0.006 & 0.00 & 0.00 & 0.00 & & 0.00 \\
\hline $\mathrm{Na}_{2} \mathrm{O}$ & 8.397 & 14.226 & 76.06 & 58.40 & 9.59 & 10.65 & 13.91 \\
\hline $\mathrm{Nd}_{2} \mathrm{O}_{3}$ & 0.680 & 0.345 & 0.00 & 0.00 & 0.64 & & 0.22 \\
\hline $\mathrm{NiO}$ & 2.489 & 0.024 & 1.16 & 0.09 & 2.24 & & 0.76 \\
\hline $\mathrm{P}_{2} \mathrm{O}_{5}$ & 1.499 & 0.240 & 0.05 & 0.00 & 1.36 & & 0.46 \\
\hline $\mathrm{PbO}$ & 0.290 & 0.718 & 0.51 & 0.00 & 0.33 & & 0.11 \\
\hline $\mathrm{PdO}$ & 0.000 & 0.159 & 0.00 & 0.00 & 0.02 & & 0.01 \\
\hline $\mathrm{Rh}_{2} \mathrm{O}_{3}$ & 0.000 & & 0.00 & 0.00 & 0.00 & & 0.00 \\
\hline
\end{tabular}




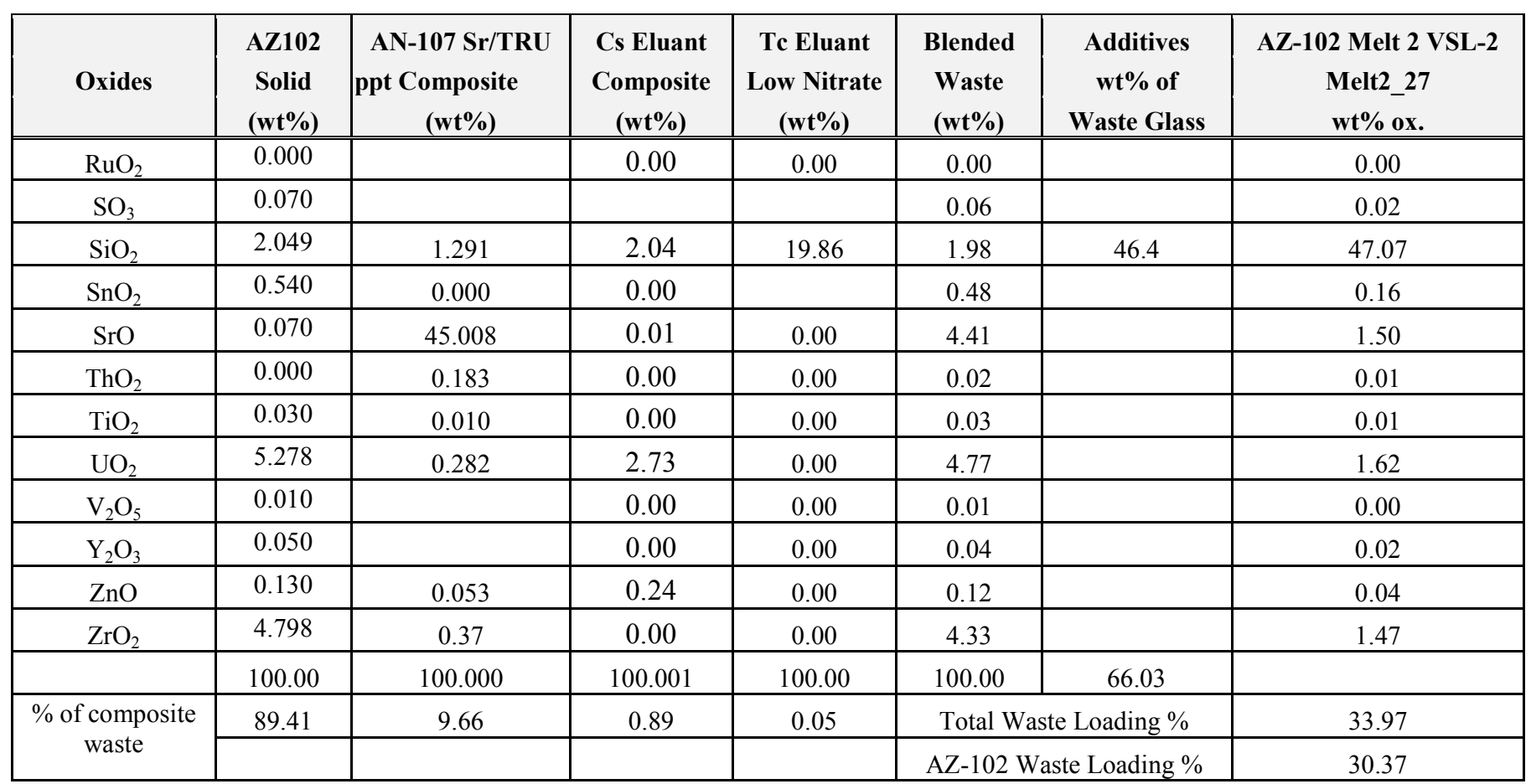

Table 3.13. AZ-102 Melt 2 Pretreated Waste and Secondary Waste Additions to Melter Feed

\begin{tabular}{|c|c|}
\hline Waste and Secondary Waste & AZ-102 Melt 2 \\
\hline \hline AZ-102 Pretreated Waste Slurry & $273.94 \mathrm{~g}$ slurry \\
\hline AN-107 Sr/TRU Precipitate & $6.47 \mathrm{~g}$ wet solids \\
\hline Tc Eluant - low nitrate & $48.19 \mathrm{~g}$ eluant \\
\hline Cs Eluant - Average & $86.50 \mathrm{~g}$ eluant \\
\hline $\begin{array}{l}\text { Note: Density of Tc eluant is assumed to be } 1.00 \mathrm{~g} / \mathrm{mL} \text {. Density of composite Cs eluants is } \\
\text { assumed to be } 1.015 \mathrm{~g} / \mathrm{mL} \text {. }\end{array}$ \\
\hline
\end{tabular}

Table 3.14. Grams of Mineral Additives added to the Waste Slurry to Make up $69.81 \mathrm{~g}$ of Glass from the AZ-102 Melt 2 Melter Feed

\begin{tabular}{|c|c|c|}
\hline Oxides & Additives & AZ-102 Melt 2 \\
\hline \hline $\mathrm{B}_{2} \mathrm{O}_{3}$ & $\mathrm{Na}_{2} \mathrm{~B}_{4} \mathrm{O}_{7} \cdot 10 \mathrm{H}_{2} \mathrm{O}$ & $6.76 \mathrm{~g}$ \\
\hline $\mathrm{Li}_{2} \mathrm{O}$ & $\mathrm{LiOH} \cdot \mathrm{H}_{2} \mathrm{O}$ & $9.74 \mathrm{~g}$ \\
\hline $\mathrm{Na}_{2} \mathrm{O}$ & $\mathrm{Na}_{2} \mathrm{SiO}_{3} \cdot 5 \mathrm{H}_{2} \mathrm{O}$ & $20.15 \mathrm{~g}$ \\
\hline $\mathrm{SiO}_{2}$ & $\mathrm{SiO}_{2}(\mathrm{Sil}-\mathrm{co}-\mathrm{Sil} 75)$ & $24.94 \mathrm{~g}$ \\
\hline $\mathrm{Sucrose}$ & Sugar & $0.84 \mathrm{~g}$ \\
\hline
\end{tabular}

\section{HLW Process Blank (AZ-102 Melt 1 Simulant) Melter Feed Batching}

This simulant melt test was conducted to provide qualitative data on the affect of the sugar-nitrate exothermic reaction on the processability of the AZ-102 Melt 2 melter feed. The results of this test provided the basis for procedure modifications to assure successful melting of the AZ-102 Melt 2 melter 
feed. This simulant melt was also produced to provide a HLW process blank as a baseline for the organic evaluations of the HLW glasses.

The AZ-102 pretreated sludge waste composite containing AN-107 Sr/TRU Precipitate was simulated by shimming available HLW pretreated sludge simulant to the AZ-102 Melt 1 melter feed composition. Table 3.15 summarizes the make up of the simulant from a pre-existing West Valley Demonstration Project (WVDP) hydroxide based waste simulant and oxides or equivalent chemicals. The WVDP simulant provided a well-homogenized mix on which to base the simulant. The need for the large proportion of added oxides was due to the high content of strontium in the WVDP simulant. The table also gives the target composition based on the AZ-102 Melt 1 feed with neodymium substituted for uranium.

Table 3.15. AZ-102 Melt 1 Simulant Used to Produce the HLW Process Blank Glass. Note that the AZ-102 Melt 1 Composition has Been Adjusted for the Substitution of Neodymium Oxide for Uranium Oxide on a Molar Basis

\begin{tabular}{|c|c|c|c|c|}
\hline & WVDP OH based simulant & Added Oxides & $\begin{array}{l}\text { Target AZ-102 Melt } 1 \\
\text { simulant composition }\end{array}$ & AZ-102 Melt 1 Simulant \\
\hline Oxide & Grams Oxide per 100gTO & Grams & Grams Oxide per 100gTO & Grams Oxide per 100gTO \\
\hline$\overline{\mathrm{Al}_{2} \mathrm{O}_{3}}$ & 8.31 & 39.00 & 7.75 & 7.73 \\
\hline $\mathrm{B}_{2} \mathrm{O}_{3}$ & 0.00 & 24.50 & 4.01 & 4.00 \\
\hline $\mathrm{CaO}$ & 1.51 & 1.50 & 0.48 & 0.49 \\
\hline $\mathrm{CdO}$ & 0.00 & 8.50 & 1.41 & 1.39 \\
\hline $\mathrm{CeO}_{2}$ & 0.00 & 0.35 & 0.06 & 0.06 \\
\hline $\mathrm{Cr}_{2} \mathrm{O}_{3}$ & 0.00 & 0.50 & 0.09 & 0.08 \\
\hline $\mathrm{CuO}$ & 0.00 & 0.20 & 0.03 & 0.03 \\
\hline $\mathrm{Fe}_{2} \mathrm{O}_{3}$ & 36.06 & 39.00 & 12.25 & 12.27 \\
\hline $\mathrm{K}_{2} \mathrm{O}$ & 3.07 & 0.00 & 0.03 & 0.50 \\
\hline $\mathrm{La}_{2} \mathrm{O}_{3}$ & 0.00 & 1.80 & 0.30 & 0.29 \\
\hline $\mathrm{Li}_{2} \mathrm{O}$ & 0.00 & 30.70 & 5.04 & 5.02 \\
\hline $\mathrm{MgO}$ & 0.24 & 0.45 & 0.12 & 0.11 \\
\hline $\mathrm{MnO}$ & 2.91 & 2.80 & 0.93 & 0.93 \\
\hline $\mathrm{Na}_{2} \mathrm{O}$ & 11.76 & 67.00 & 13.38 & 12.87 \\
\hline $\mathrm{Nd}_{2} \mathrm{O}_{3}$ & 0.00 & 7.50 & 1.23 & 1.23 \\
\hline $\mathrm{NiO}$ & 1.45 & 3.30 & 0.77 & 0.78 \\
\hline $\mathrm{P}_{2} \mathrm{O}_{5}$ & 0.00 & 3.00 & 0.47 & 0.49 \\
\hline $\mathrm{PbO}$ & 0.00 & 0.50 & 0.09 & 0.08 \\
\hline $\mathrm{SiO}_{2}$ & 22.67 & 274.00 & 48.39 & 48.50 \\
\hline $\mathrm{SnO}_{2}$ & 0.00 & 1.00 & 0.17 & 0.16 \\
\hline $\mathrm{SrO}$ & 9.09 & 0.00 & 1.50 & 1.49 \\
\hline $\mathrm{TiO}_{2}$ & 0.01 & 0.04 & 0.01 & 0.01 \\
\hline $\mathrm{ZrO}_{2}$ & 2.92 & 6.10 & 1.48 & 1.47 \\
\hline & 100.00 & 511.74 & 100.00 & 100.00 \\
\hline
\end{tabular}




\section{HLW Melter Feed Preparation and Glass Fabrication Summary}

Exact details of how each waste was processed and melted will be discussed in Section 4.0. This subsection will outline the process used for feed processing and batch melts.

Pretreated tank wastes (see Figure 3.3 for an example) were vigorously blended in a stainless steel beaker using a magnetic stirrer and stir bar. A peristaltic pump was used to subdivide wastes for physical and rheological testing, slurry preparation, and sample archival. Secondary wastes (Sr/TRU precipitate, the composite Cs ion exchange eluant, and Tc ion exchange eluants) were combined with the pretreated tank sludge waste into the same stainless steel beaker. The glass forming mineral additive mixture was then added to complete the melter feed.

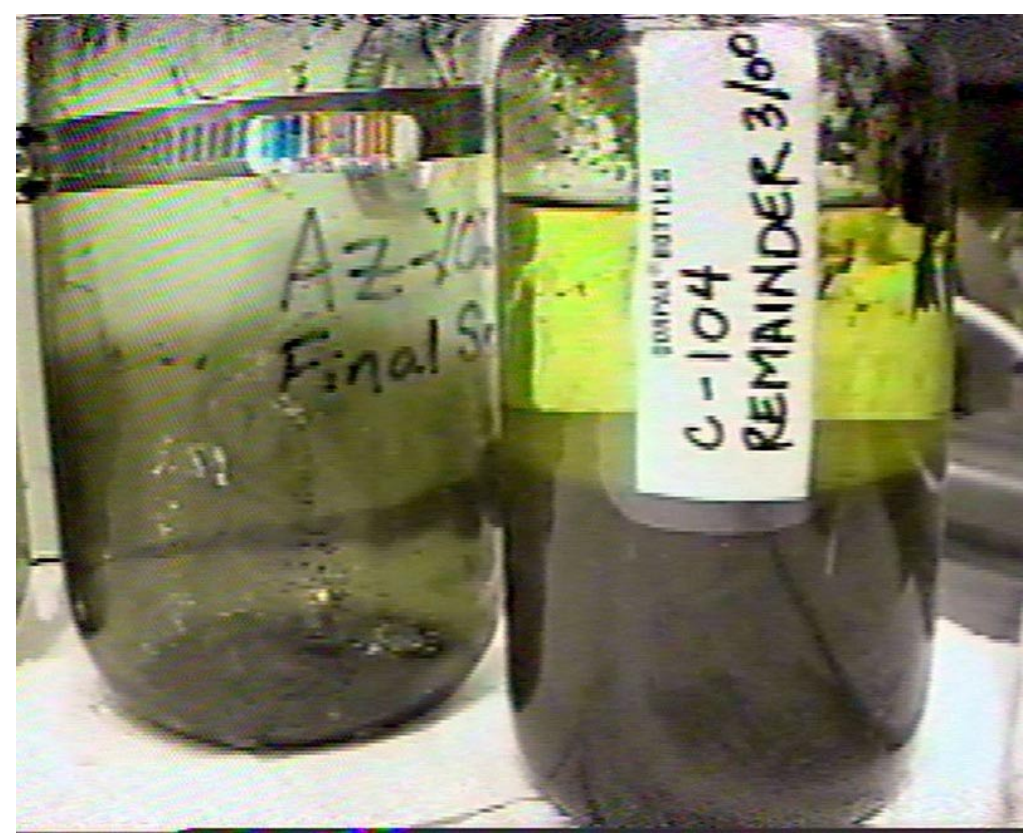

Figure 3.3. The pretreated C-104 tank waste archived sample. This photo gives a good view of the settled waste with the yellow supernate segregated from the solids. Next to the C-104 waste is the empty storage container for the pretreated AZ-102 tank waste, which has a high dose rate and left the glass container darkened where the waste solids had settled.

The melter feed slurry was heated and stirred on a hot plate to evaporate water. Mixing was vigorous so solids from the mineral additives could not settle. The heating / stirring process took three to five hours to thicken the batch to the point the stir bar would no longer rotate. Hand blending using manipulators continued while the melter feed sample was heated on the hot plate until the batch formed a dry crust and eventually completely dried; this took an additional one to three hours to complete. The dry cake that was produced was hard and brittle. The blended and dried feed was then added to a $300 \mathrm{~mL}$ Pt-10\% Rh crucible, placed into a furnace at approximately $600^{\circ} \mathrm{C}$ and calcined for about 4 hours. 


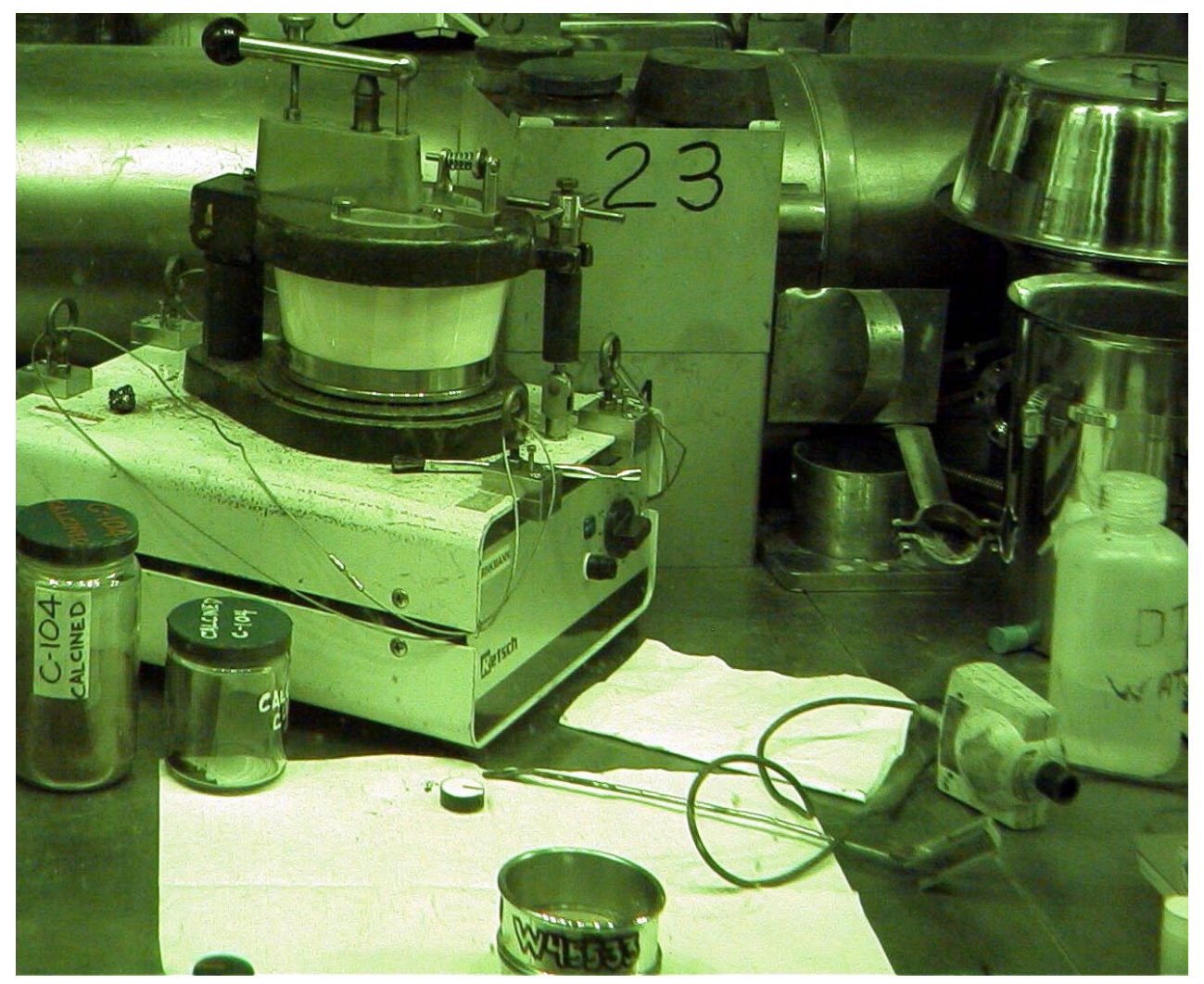

Figure 3.4. The automated grinder it shown in the center-left of the picture with a sintered aluminum oxide mortar and pestle clamped between the two black metal supports atop the machine. The top metal support is removable so that the pestle can be taken out to remove the crushed sample. A stack of 3-inch diameter stainless steel sieves is in the foreground, jars of processed feed are adjacent to the grinder, and the in-cell video camera is lying on its side next to the poly water dispenser.

The calcined batch was crushed in an automated alumina mortar and pestle (see Figure 3.4) and passed through a 40 mesh sieve. About half the powder was added back into the Pt $10 \% \mathrm{Rh}$ crucible melted in a high temperature furnace for about twenty minutes and the remainder of the batch added to the melt. The batch melted approximately 2 hours with a lid covering the crucible. The glass melt was then poured onto a stainless steel plate (air quenched), cooled to room temperature, and handled in a manner to keep the glass free of organic contamination. All glass samples were stored in glassware cleaned to EPA standards. (These jars were purchased from VWR and came from the manufacturer (I-Chem) with a certificate that states "product meets or exceeds analyte specifications established in the US EPA Specification and Guidance for Contaminant-free Sample Containers." The product was ordered as a Group 3 Type container: cleaned for the use of the container for the analysis of volatiles.) A portion of the melt was poured into a small box crucible, about $20 \mathrm{~mL}$, (see Figure 3.5) to be heat treated following the predicted canister centerline cooling (CCC) heat treatment of a Hanford HLW canister of glass $3 / 5$ of the way from the bottom of the canister (see Section 3.2 for details).

Vitrification of slurry melter feed in an actual melter progresses continuously through 3 distinct stages, drying, calcining and melting. Under steady-state operating conditions, the aqueous slurry that is introduced into the high-temperature melter environment spreads out over an existing cold cap where it dries and becomes part of the melter cold-cap structure. This dried material begins working it's way down through the cold-cap as it becomes submerged in incoming feed while, at the same time, material, at 
the molten-glass/cold-cap interface, is dissolving into the glass melt. During this continuous progression through the cold cap, the temperature that the feed is subjected to monotonically increases from the boiling point of water $\left(\sim 100^{\circ} \mathrm{C}\right)$ to molten glass temperatures $\left(\sim 1150^{\circ} \mathrm{C}\right)$. Accompanying this continuous physical and thermal transition, inorganic eutectic salts are slowly converted to their oxide forms (calcined) that are suitable for subsequent incorporation into the melter's molten glass pool.

All of these discrete phases of liquid-fed ceramic melter (LFCM) feed processing have been faithfully reproduced in the crucible studies performed. What may not be truly represented, however, is the complex stages and nature of the cold-cap chemistry that results in the calcination of the feed material. For non-volatile, inorganic feed constituent, the differences between crucible and melter vitrification conditions are inconsequential. For all other feed components, cold-cap chemistry can influence both partitioning behavior and chemical byproduct yields, which, in turn, can and will affect the resultant glass product.

Consequently to properly represent an LFCM glass product, actual physical and chemical processing conditions need to be replicated. But since this requires the development of a representative cold-cap structure, nothing short of a liquid-fed melting process (e.g., scaled melter or possibly a gradient furnace test) is truly adequate. However, relationships drawn between previous crucible and actual melter testing results, i.e. from VSL and GTS Duratek testing, that were conducted using a fixed feed may be useful in extracting reference glass-product quality parameters from extrapolated crucible test data.

\section{$\underline{\text { Test Equipment }}$}

The Envelope D radioactive, pretreated sludge and secondary wastes, and additives feed were dried in a Blue-M Stabil-Therm Gravity drying oven, and calcined and melted in a custom-made Del Tech high-temperature bottom loading furnace equipped with a Eurotherm programmer/controller and the temperature monitored with a calibrated Type K thermocouple and an Omega, Model 660 thermocouple readout. Vitrification was completed in a $300-\mathrm{mL}$ platinum $/ 10 \%$ rhodium crucible.

A Reisch automated mortar and pestle grinding mill with an alumina grinding chamber was used to crush and mix the glass, and 3-in.-diameter stainless-steel sieves were used to sieve glass samples.

\subsubsection{Chemical Composition}

Chemical composition of the three HLW glasses (i.e., elements \{excluding oxygen\}) present in concentrations greater than 0.5 percent by weight) were measured in duplicate along with an analytical reference glass-1 (ARG-1) powdered glass reference standard (Smith 1993) using a sodium peroxide $\left(\mathrm{Na}_{2} \mathrm{O}_{2}\right)$ fusion, according to procedure PNL-ALO-114, and a potassium hydroxide (KOH) fusion, according to procedure PNL-ALO-115. Analytical Reference Glass-1 (ARG-1) is a compositionally well-characterized glass and provides an excellent independent check of the analytical processes and results. The $\mathrm{KOH}$ fusion uses a nickel crucible and the $\mathrm{Na}_{2} \mathrm{O}_{2}$ fusion uses a zirconium crucible. Cation analysis was performed using Inductively Coupled Plasma-Atomic Emission Spectrometry (ICP-AES). Approximately 0.1 grams of sample was processed and diluted to a final volume of about $100 \mathrm{ml}$ (the final solution volume was weighed and density corrected to a volume). Hydrofluoric acid was added as necessary to ensure 'clear' solutions. All sample material after processing appeared to go into solution (no apparent residue remained in fusion crucibles or as precipitate in final solution). Analytical dilution of 2 and 10-fold were prepared for each fusion preparation and analyzed by ICP-AES. An analytical process blank (not to be confused with the AZ-102 Melt 1 simulant glass HLW Process Blank) was prepared similarly at the same time as the above samples. 
A portion of the PNL-ALO-114 (sodium peroxide) fusion prepared samples was submitted for radiochemical analysis and Inductively Coupled Plasma-Mass Spectrometry (ICP-MS) analysis (see Section 3.1.3 Radiochemical Composition). No hydrofluoric acid was added to the aliquots submitted for radiochemistry or ICP-MS analysis.

\section{$\underline{\text { Test Equipment }}$}

Cation analysis of the leachate solutions was completed using a Thermo Jarrell-Ash, Model 61 inductively coupled argon plasma spectrometer according to procedure PNL-ALO-211.

\subsubsection{Radiochemical Composition}

Radiochemical analyses were performed on two of the IHLW glass products, i.e. C-104 and AZ-102 Melt1 glasses. Analyses included ${ }^{60} \mathrm{Co},{ }^{106} \mathrm{Ru},{ }^{125} \mathrm{Sb},{ }^{134} \mathrm{Cs},{ }^{137} \mathrm{Cs},{ }^{144} \mathrm{Ce},{ }^{154} \mathrm{Eu},{ }^{155} \mathrm{Eu}$, and ${ }^{241} \mathrm{Am}$ by GEA, ${ }^{90} \mathrm{Sr},{ }^{239 / 240} \mathrm{Pu},{ }^{241} \mathrm{Pu}$, and total uranium. Concentration values of additional gamma emitters (i.e., ${ }^{51} \mathrm{Cr},{ }^{59} \mathrm{Fe},{ }^{79} \mathrm{Se},{ }^{95} \mathrm{Nb},{ }^{103} \mathrm{Ru},{ }^{113} \mathrm{Sn}$, and ${ }^{152} \mathrm{Eu}$ ) were obtained by GEA depending on concentrations and detection limits. The following radioisotopes: ${ }^{99} \mathrm{Tc},{ }^{237} \mathrm{~Np},{ }^{239} \mathrm{Pu},{ }^{240} \mathrm{Pu},{ }^{233} \mathrm{U},{ }^{234} \mathrm{U},{ }^{235} \mathrm{U},{ }^{236} \mathrm{U}$ and ${ }^{238} \mathrm{U}$ were measured by inductively coupled plasma mass spectroscopy (ICP-MS) in the C-104, AZ-102 Melt1, and AZ-102 Melt2 glasses.

Samples of waste glass, C-104 and AZ-102 Melt 1, were initially processed in the shielded analytical laboratory (SAL). A nominal $0.1 \mathrm{~g}$ sample was fused using $\mathrm{Na}_{2} \mathrm{O}_{2}$ flux according to PNL-ALO-114, and brought to a $100-\mathrm{mL}$ volume. The samples were prepared in duplicate with a process blank. Aliquots of these solutions were distributed to the radiochemistry laboratory for subsequent radiochemical analyses.

Aliquots from the SAL-prepared solutions were prepared for gamma spectrometry in a standard geometry and counted using high-purity germanium (HPGe) detectors according to PNL-ALO-450. Aliquots of the diluted SAL preparation were taken for ${ }^{90} \mathrm{Sr}$ analysis. Strontium was isolated according to procedure PNL-ALO-476, beta-counted according to PNL-ALO-408, and the ${ }^{85} \mathrm{Sr}$ tracer was gamma-counted according to PNL-ALO-450. Along with the samples, a chemistry sample replicate (00-2313Rep), a chemistry blank (Blank), a reagent spiked with ${ }^{90} \mathrm{Sr}$ (Blank Spike), and a sample spiked with ${ }^{90} \mathrm{Sr}$ (Matrix Spike) were also prepared. These QC samples help verify the accuracy of the sample separation, counting, and analysis methods.

Plutonium was isolated from diluted SAL preparations according to PNL-ALO-417. The separated fractions were then precipitation plated according to PNL-ALO-496 and counted by alpha spectrometry according to PNL-ALO-422. After alpha-counting was complete, the filters were suspended in scintillation cocktail and beta-counted according to PNL-ALO-474. The ${ }^{241} \mathrm{Pu}$ count rate was determined by integrating from $2-20 \mathrm{keV}$. The liquid scintillation counter was calibrated relative to tritium that has a similar beta end-point energy $(18.6 \mathrm{keV})$.

Total uranium was determined on dilutions of the SAL preparations using kinetic phosphorescence analysis according to PNL-ALO-4014.

In addition to the above analyses, C-104, AZ-102 Melt1, and AZ-102 Melt2 glass samples were analyzed by ICP-MS for technetium, uranium, and plutonium isotopic analysis. 
Test Equipment

Test equipment conformed to that required to carry out the PNL-ALO and RPG-CMC procedures called out above.

\subsection{Crystalline and Non-Crystalline Phase Determination}

Crystalline and non-crystalline phases were identified and measured using x-ray diffraction (XRD), optical microscopy, and scanning electron microscope (SEM) on glass samples of C-104 and AZ-102 Melt 1 that had been heat-treated to simulate a HLW canister centerline cooling (CCC) curve. In addition, CCC heat-treated C-104 and AZ-102 Melt 1 glass samples were used to prepare the powder samples for PCT durability testing (see Section 3.3 below).

\section{$\underline{\text { Canister Centerline Cooling Heat-treatment }}$}

Samples of two HLW glasses (C-104 and AZ-102 Melt 1) were given a slow cool down heat treatment which simulates the cooling profile for glass at the center line of a Hanford HLW canister being filled with a waste glass and allowed to cool to ambient temperature. The immobilized high-level waste (HLW) stainless steel canisters are basically right circular cylinders $4.5 \mathrm{~m}$ in height and $0.61 \mathrm{~m}$ in diameter. Glass canister filling was modeled with a batch target fill rate of $500 \mathrm{~kg} / \mathrm{hr}$ for 30 minutes at a temperature of $1150^{\circ} \mathrm{C}$ with 3 hours between pours (12 MT/day). Based on the canister configuration and fill rate, BNFL provided a model calculation of the cooling curve for the centerline of a canister of glass $3 / 5$ of the way from the bottom of the canister. This model curve was approximated by a series of linear time-temperature segments that a programmable furnace duplicated. Table 3.16 below gives the set of linear time-temperature segments, which were duplicated by the furnace to within \pm 4 to $5{ }^{\circ} \mathrm{C}$ at all points along the profile.

Table 3.16. Temperature Profile Line Segments Used as Guidelines for Programming the Del Tech Furnace Controller to Generate the Hanford HLW Canister Centerline Cooling Profile

\begin{tabular}{|c|c|c|}
\hline Hours & Temperature $\left({ }^{\mathbf{}} \mathbf{C}\right)$ & dT/dt(deg./hr) \\
\hline $0.00-0.17$ & $1004.2-1050.4$ & +277.2 \\
\hline $0.17-2.17$ & $1050.4-1002.5$ & -23.95 \\
\hline $2.17-7.0$ & $1002.5-843.7$ & -32.86 \\
\hline $7.0-10.3$ & $843.7-749.2$ & -28.63 \\
\hline $10.3-15.5$ & $749.2-617.4$ & -25.35 \\
\hline $15.5-21.17$ & $617.4-490.8$ & -22.40 \\
\hline $21.17-25.8$ & $490.8-399.8$ & -19.65 \\
\hline
\end{tabular}

The furnace used to do the model CCC heat treatment was the same high temperature Del Tech furnace used to melt the glasses. The heat treated samples consisted of about 30 to 35 grams of glass which was poured from the initial melt into a $2.5 \mathrm{~cm}^{3}$ crucible of platinum, $10 \%$ rhodium foil with a tightly fitted lid covering the crucible. These samples were returned to the furnace (Figure 3.5) as soon as it was ready to run the CCC profile. The samples were heat-treated, cooled to ambient hot cell temperature, and removed from the furnace, and then the samples were weighed, taken out of their crucibles and stored in labeled glass jars. 
After the CCC heat treatment, each sample was cut into three to four sections perpendicular to the melt surface with a diamond wafering low-speed saw (Figures 3.6 and 3.7). The largest piece, about 11 grams from each glass sample, was used to prepare the powder samples for PCT durability testing and a smaller corner section was used to make a thin section (a cross-sectional slice) for optical and scanning electron microscopy.

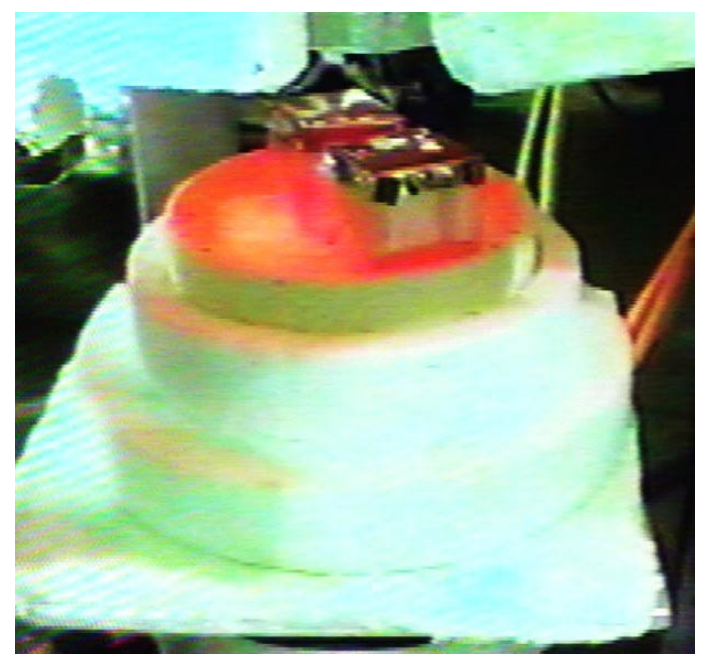

Figure 3.5. Samples of C-104 and AZ-102 glass in platinum 10\% rhodium box crucibles covered with lids are placed on the hot hearth plate for the CCC heat treatment. The pedestal that holds the hearth plate will be hydraulically lifted into the heating cavity of the high temperature furnace.

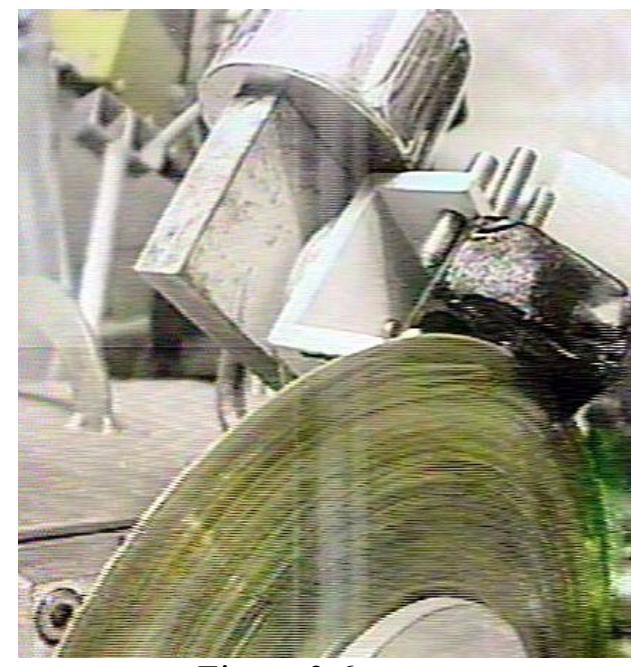

Figure 3.6

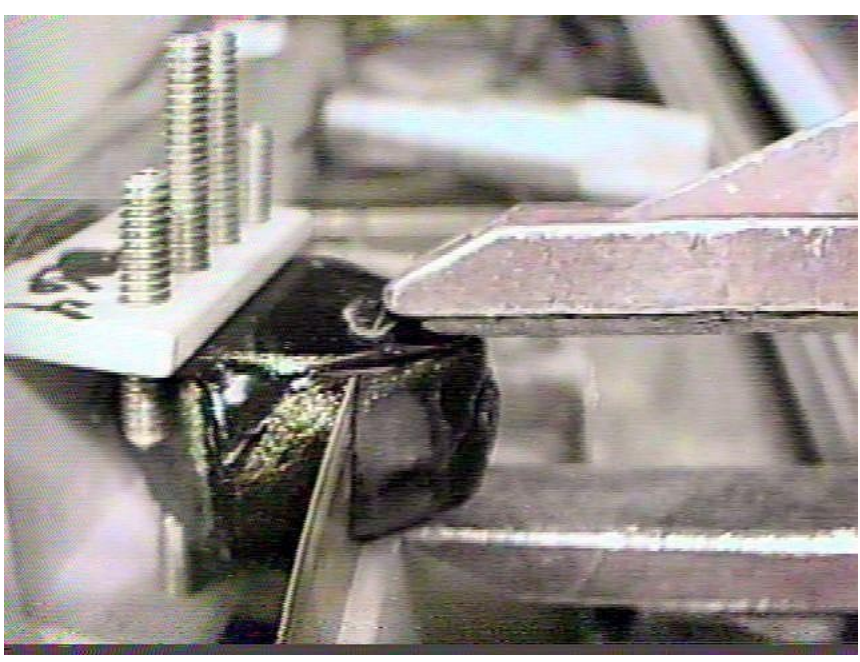

Figure 3.7

Figure 3.6. Cutting process for preparation of the AZ-102 CCC heat-treated glass samples showing the beginning cut using a diamond wafering blade.

Figure 3.7. Cutting process for preparation of the AZ-102 CCC heat-treated glass samples showing the final sectioning stage where the manipulator fingers are grasping the section of glass just about to be separated from the original sample. 
$\underline{X-r a y ~ D i f f r a c t i o n ~ A n a l y s i s ~ S a m p l e ~ P r e p a r a t i o n ~}$

For XRD analysis, a piece of CCC heat treated glass was broken from a segment of sectioned glass, crushed in an alumina mortar and pestle grinder, sieved through a 75 micron (200 mesh) sieve and stored in a glass vial until analysis. The powder prepared for XRD analysis was weighed to between 8 and $9 \mathrm{mg}$, mixed into a solution of callodin, mounted on a plastic XRD sample mount, leveled to X-ray beam height, encapsulated in Mylar film, and transported to the XRD laboratory for analysis. The twotheta scan range was from 5 to 75 degrees at a step size of 0.05 degrees with a 1 second dwell at each step.

\section{Optical and Scanning Electron Microscopy Sample Preparation}

For optical and scanning electron microscopy, a thin slice of one of the cross sections of the CCC heat-treated glass was used. Conventional grinding and polishing techniques were followed. A section of glass was ground and polished to a 600 grit surface finish, mounted to a glass slide with super glue, cut to a thin cross section, and the exposed surface of the sample ground and polished until it had a mirror reflection (final polish was a 6 micron polishing paste). The thickness of the glass thin section was estimated to be $<0.25 \mathrm{~mm}$.

The thin slices of C-104 and AZ-102 glass were examined using an optical microscope in both reflected and transmitted light (magnification from 50 to 200×). The analysis was accomplished in Mini Hot Cell 7 in the Shielded Analytical Laboratory located in the RPL facility. Viewing of the samples was accomplished with a video camera with the image viewed on a monitor near the hot cell.

When preparations were being made to do analysis by SEM, it was determined that the radiological dose rates on the samples were too high for the operating conditions of the SEM in the 326 Building radioactive SEM facility. Size reduction of the samples was necessary to cut the dose and the samples were broken to obtain smaller samples. From the AZ-102 sample a sliver about $2 \times 5$ to $6 \mathrm{~mm}$ and from the C-104 sample, a piece about $5 \mathrm{~mm}^{2}$, were used for analysis. The samples were bonded to new glass slides with super glue, the surface of the glass decontaminated with methanol and the samples shipped for analysis. Each slide was then coated with a gold film and examined at low magnification $(100 \times)$ and higher magnifications of $500 \times, 1500 \times, 2000 \times$, and $5000 \times$.

$\underline{\text { Testing and Test Sample Evaluation Equipment }}$

Glass samples were cut and polished with Buehler diamond saw and polishing equipment modified for handling with hot cell manipulators. Optical microscopy was completed using a Conneaut Lake Scientific microscope. An SEM (Model VG Elemental Shielded PQ2) with EDS capability was used to look for crystals and chemical inhomogeneities. XRD was performed using a Scintag X-ray diffractometer, model PAD V, employing $\mathrm{Cu} \mathrm{K"} \mathrm{radiation} \mathrm{(1.54056} \mathrm{C).}$

\subsection{Release Rate, Product Consistency Testing of HLW Glasses}

The ultimate objective for immobilization of the high-level radioactive tank waste is to incorporate and convert the radioactive and hazardous components into a solid waste form that will be chemically durable and meet the conditions for storage in a geologic repository for high-level radioactive waste. This resistance of the waste form to release deleterious environmental components is defined by measuring its chemical durability, i.e. the resistance of the glass to react with the aqueous environment expected in the glass disposal site. However, to mimic the mean temperature, amount and frequency of available ground waster, etc. expected in the geologic repository would require a great amount of testing 
time to be able to detect glass dissolution. Therefore, an accelerated chemical durability test, the Product Consistency Test (PCT), is employed to gauge the IHLW glass chemical durability. The glass samples used in the PCT were given a slow cool-down heat treatment (see Section 3.2 for details) which simulates the cooling profile for glass at the center line of a Hanford standard HLW canister being filled with a waste glass and allowed to cool to ambient temperature. The PCT was run at $90^{\circ} \mathrm{C}$ to determine the normalized release of boron, sodium, lithium, aluminum, and silicon. The Environmental Assessment glass (EA glass) test reference material, standard glass (Jantzen et al. 1993) was included in these tests to provide a reliable baseline of results by which to judge the quality of the PCT results for the C-104 and AZ-102 Melt 1 glasses.

The ASTM procedure C 1285-97 "Standard Test Methods for Determining Chemical Durability of Nuclear, Hazardous, and Mixed Waste Glasses: The Product Consistency Test (PCT)"was used to test the durability of C-104, AZ-102 Melt 1, and the EA glasses. A brief summary of the steps followed is provided here. Each glass was ground in an automated alumina mortar and pestle (grinding chamber) and then sieved through 75 and $150 \mu \mathrm{m}(-100$ to +200 mesh) stainless steel sieves. The glass particles were cleaned by washing in deionized water (DIW) and ethanol using an ultrasonic cleaner, and dried in an oven at $90^{\circ} \mathrm{C}$ as per the PCT procedure. Approximately $1.5 \mathrm{~g}$ of glass was weighed and placed into a 22 $\mathrm{mL}$ desensitized Type 304L stainless steel container (See Figure 3.8). The volume of water for each sample was measured by mass and added to the requisite stainless steel container. The glass was precisely weighed and the leachant volume precisely controlled to achieve a solution volume to glass mass ratio of $10 \mathrm{~mL} / \mathrm{g}$ glass. The ratio of the surface area of the sample to the leachant volume is calculated to be about $2000 \mathrm{~m}^{-1}$. To calculate the exposed glass surface area for the $-100(0.149 \mathrm{~mm})$ to $+200(0.074 \mathrm{~mm})$ mesh glass particles the glass particles are assumed to be spherical and that the particle size distribution over this range is Gaussian (shown to be true based upon particle size distribution measurements provided in Appendix X1 of ASTM C 1285-97). (Note that doing the same analysis assuming cubic or tabular particles results in a surface area number that differs from that obtained using spherical particles by about $1 \%$.) As such, using the average diameter of well sieved samples in the -100 to +200 mesh range introduces no significant error to calculating the exposed glass surface area. Based upon these assumptions one can calculate the average particle size and mass that enables one to calculate the number of particles per gram of crushed glass and finally the total calculated exposed glass surface area. Triplicate samples were prepared and tested for each of the three glasses. Two vessels were tested as blanks by filling the container with $15 \mathrm{~mL}$ of DIW and following the PCT procedure except no glass sample was added to the vessel. Each container and their contents were held (without agitation) at $90^{\circ} \mathrm{C}$ for 7 days for each PCT conducted with each glass sample. The initial and final $\mathrm{pH}$ values of the solution were taken. Aliquots of the solution were filtered through a $0.45-\mu \mathrm{m}$ filter and submitted for ICP analysis. Results are reported as normalized elemental mass releases in Section 4.3.

All tests were run without deviation from the procedure described above except the duration of time was short by 0.5 percent at the test temperature of $90^{\circ} \mathrm{C}$. The requirement for the test is $\pm 2 \%$ of the seven day test period, which is $168 \pm 3.4$ hours. All glass samples were within $2.5 \%$ of the test period. A deficiency report has been filed, and further details of this deviation are explained in Section 4.3. 


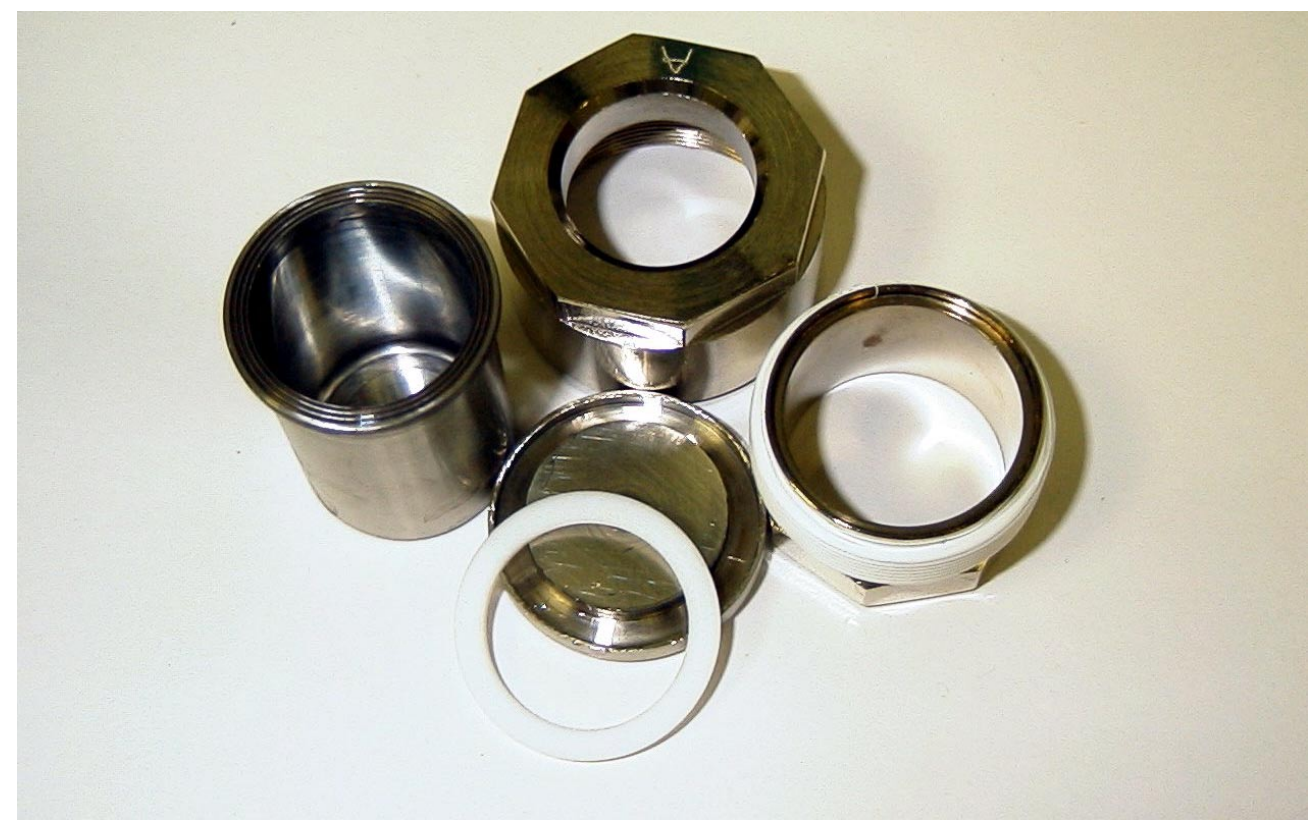

Figure 3.8. Pictured are the Desensitized Type 304L stainless steel, $22 \mathrm{~mL}$, PCT vessel and lid, a white Teflon gasket which seals the vessel and lid when the assembly is closed and tightened, and the nickelplated brass, nut and screw vessel tightening assembly.

\section{$\underline{\text { Test Equipment }}$}

Leach vessels used were 22-mL screw-cap containers fabricated from desensitized 304L stainless steel. The vessels, including the lids and Teflon gaskets, were cleaned following the ASTM C 1285-97 procedure. DIW used for cleaning and leachate was taken from a Barnstead, NANOpure Ultra Water System, Model D4741 with resistivity of the water measured at $18.1 \mathrm{M} \Omega \cdot \mathrm{cm}$. An Orion Research Ion Analyzer, Model 720A was used to measure the $\mathrm{pH}$ of solutions outside the hot cells and an Orion Research Ion Analyzer, Model 520A, was used to measure the final $\mathrm{pH}$ of the PCT solutions in the hot cell. The $\mathrm{pH}$ meters were calibrated before use with VWR brand buffer solutions of 4.00, 7.00, and 10.00. A Blue M oven in the HLRF Hot Cell C and an Omega temperature controller, Series CN7100, located in the HLRF gallery were used for the $90^{\circ} \mathrm{C}$ seven day PCT.

Analysis of the PCT leachate solutions was completed using a Thermo Jarrell-Ash, Model 61 inductively coupled argon plasma atomic emission spectrometer according to procedure PNL-ALO-211. 


\subsection{Results}

\subsection{Glass Fabrication and Analysis}

Four glass samples (C-104, AZ-102 Melt 1, AZ-102 Melt 2, and the HLW Process Blank) were successfully processed and melted into a HLW glass form. These glasses were prepared for chemical and radiochemical composition determination.

Approximately $239.8 \mathrm{~g}$ of useable, i.e. poured from crucible, C-104 glass was produced $(250 \mathrm{~g}$ theoretical). The amount of useable AZ-102 Melt 1 produced was about $115 \mathrm{~g}$ (118.9 g theoretical) and the amount of AZ-102 Melt 2 used was $35.86 \mathrm{~g}$ (produced $69.81 \mathrm{~g}$ theoretical).

\subsubsection{Glass Fabrication}

Feed Preparation and Vitrification of the C-104 Glass

The C-104 melter feed was prepared in hot cell "C" of the HLRF. A portion of the Cs ion exchange eluant composite, $529.0 \mathrm{~g}$, was placed in a $4 \mathrm{~L}$ stainless steel beaker and heated and agitated with a Teflon stir bar on a combination hot plate/stirrer. Next, $25.55 \mathrm{~g}$ of the actual AN-107 Sr/TRU precipitate was added to the solution. This solution was evaporated, then, the C-104 pretreated sludge waste $(400.5 \mathrm{~g})$ was added to this mixture. Deionized water was used to thoroughly rinse containers and wash beaker walls.

The dry mineral additives, borax, $\left(\mathrm{Na}_{2} \mathrm{~B}_{4} \mathrm{O}_{7} \cdot 10 \mathrm{H}_{2} \mathrm{O}\right)$; sodium carbonate, $\left(\mathrm{Na}_{2} \mathrm{CO}_{3}\right)$; lithium hydroxide, $\left(\mathrm{LiOH} \cdot \mathrm{H}_{2} \mathrm{O}\right)$; silica sand $\left(\mathrm{SiO}_{2}\right)$; and zinc oxide $(\mathrm{ZnO})$, were weighed, blended together for 2 minutes in a $250 \mathrm{~mL}$ agate milling chamber in the Glass Development Laboratory of APEL. From a $230.42 \mathrm{~g}$ chemical batch, $219.53 \mathrm{~g}$ was weighed, transferred into hot cell "C", and slowly added as four separate scoops into the heated waste slurry as it stirred. No visible changes were observed with the addition of the mineral additives, dissolution and suspension of the materials into the slurry went well. The slurry was heated and mixed until a sludge formed and the stir bar stopped. Agitation continued manually with manipulator stirring using a stainless steel stir rod (see Figure 4.1).

It took several days from the beginning of the evaporation process until the melter feed was dry. Heating of the melter feed occurred only when someone was monitoring the process but evaporation was allowed over night by leaving the slurry/sludge open to the $30 \pm 2{ }^{\circ} \mathrm{C}$ hot cell ambient temperature. During the first $24 \mathrm{~h}$ of drying, enough liquid was present in the slurry for continuous stirring with a magnetic stir bar. During the second day of heating the slurry thickened and manipulator stirring became necessary. By the end of the second day, the viscosity of the slurry was like the consistency of molten fudge. The heat was turned off and over night the slurry hardened. The stirring rod could not penetrate the hardened melter feed the following morning, but further drying was necessary. When the melter feed was again heated on the hot plate, it softened back into a smooth, viscous slurry.

Manipulator stirring continued for 2.5 hours. After $1.5 \mathrm{~h}$ of stirring, the melter feed clumped into clusters several centimeters in diameter, but the clumps were 'gooey'. By breaking the clusters and moving them about during the next half hour, the moisture level decreased and the clusters hardened. An additional half hour of heating continued to drive off moisture while the beaker walls were carefully scraped of residual melter feed. At this point the beaker was placed in an oven for about 1 hour at $115^{\circ} \mathrm{C}$. 


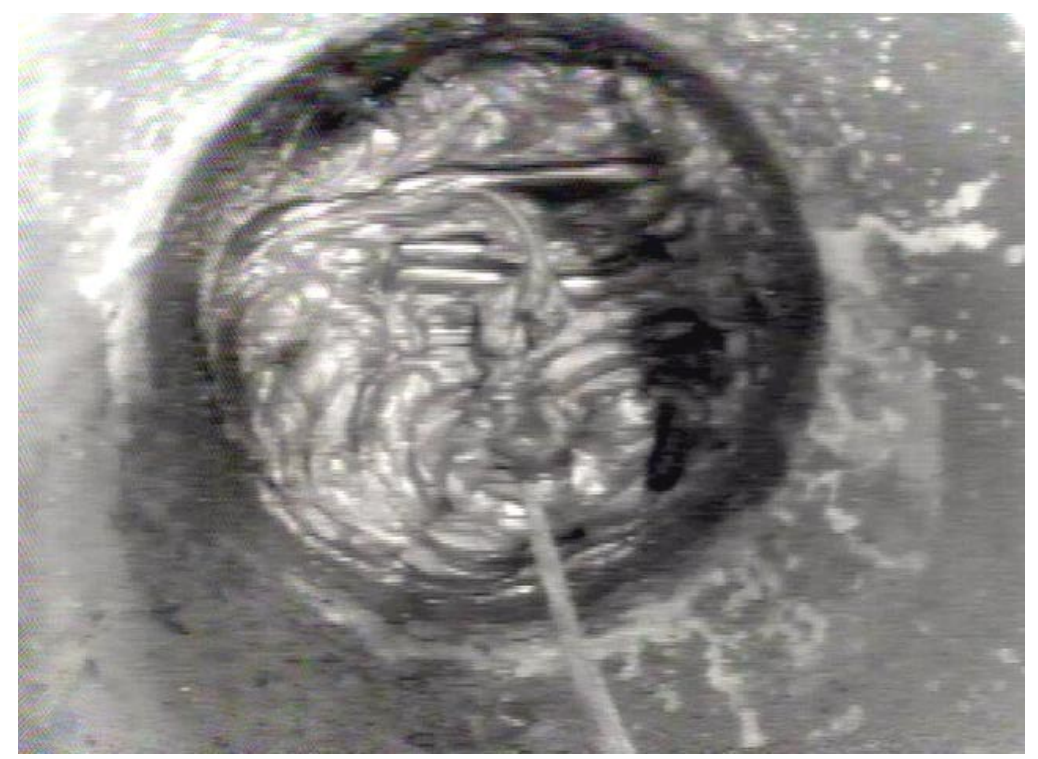

Figure 4.1. Thick C-104 melter feed sludge, at the bottom of a $4 \mathrm{~L}$ stainless steel beaker, is stirred with a stainless steel stir rod rotated by the hot cell manipulator arm.

The dry melter feed was transferred to a $300 \mathrm{~mL}$ platinum, 10\% rhodium (Pt10\%Rh) crucible (Figure 4.2) and placed into a furnace that ramped slowly from 290 to $320^{\circ} \mathrm{C}$ over $1 \mathrm{~h}$ (Figure 4.3). Drying was complete when the sample was removed from the furnace. The melter feed reduced slightly in volume during the final drying cycle, changed in color from a dark gray to areas on the sample that had light and dark brown hues. The furnace also had brown stains probably from nitrate decomposition and clear liquid was observed condensed on the top sill above the furnace door. The sample was hard and brittle, and appeared to be dry.

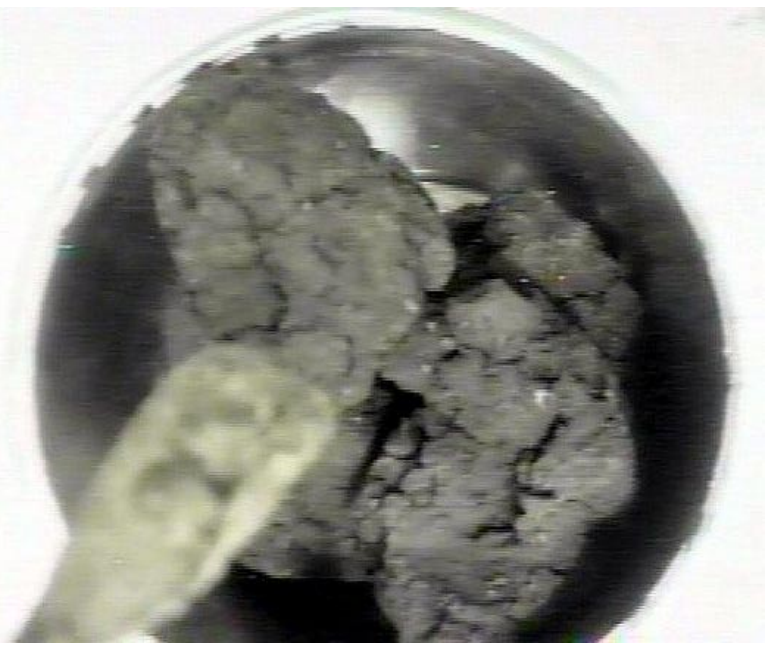

Figure 4.2

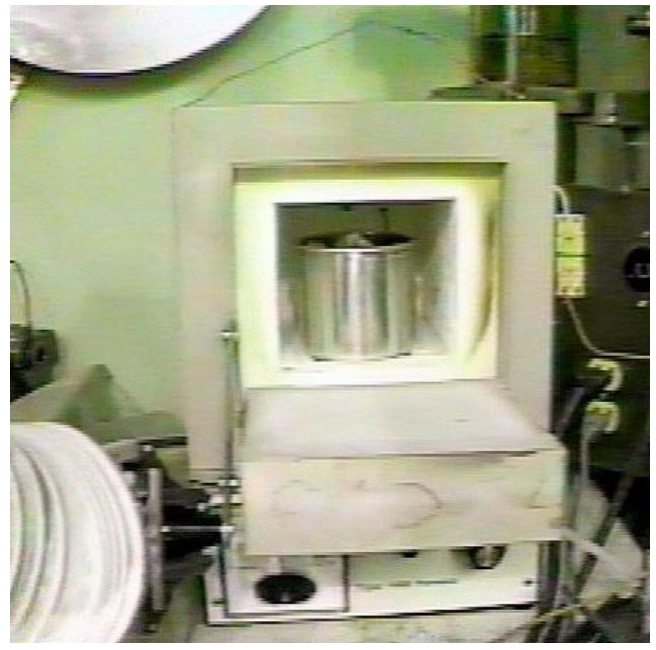

Figure 4.3

Figure 4.2. The C-104 melter feed in large chunks in the Pt10\%Rh crucible after drying on the hot plate. The tip of the stainless steel stir rod is in the foreground above the crucible.

Figure 4.3. The Thermolyne furnace used for drying and calcining the C-104 melter feed. The manipulator arm is holding the furnace door open and the Pt $10 \% \mathrm{Rh}$ crucible with dried feed is inside the furnace cavity. Note the dark stains on the white insulation surrounding the furnace cavity. This is probably from nitrate volatilizing during the drying cycle. 
The melter feed in the Pt $10 \% \mathrm{Rh}$ crucible was then placed back into the furnace at $600^{\circ} \mathrm{C}$ for about $1.5 \mathrm{~h}$ to begin the calcining process. The temperature was increased to $650^{\circ} \mathrm{C}$ and adjusted up to $681^{\circ} \mathrm{C}$ over a one hour period. A crust began to form on the surface of the chunks of melter feed, so to allow for full nitrate evolution, the temperature was lowered in the furnace to $624^{\circ} \mathrm{C}$ and calcining continued for an additional hour. Calcining was complete and the crucible was removed and cooled to ambient hot cell temperature. The rounded chunks nearly maintained their shape through the drying/calcining process (compare Figures 4.2 and 4.4).

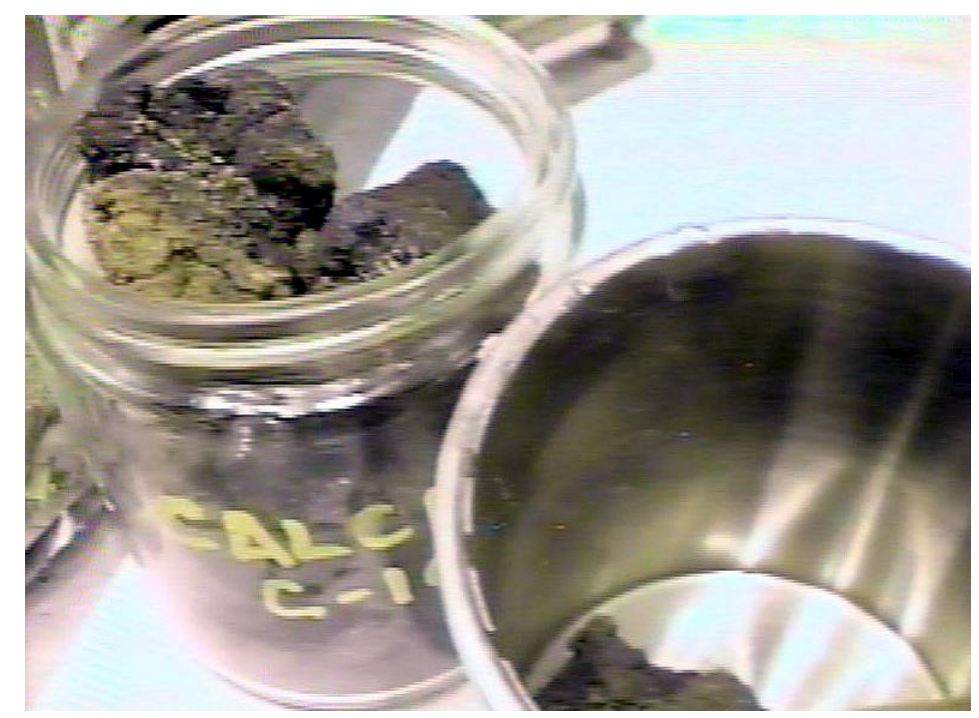

Figure 4.4. Calcined C-104 melter feed in glass jar next to the Pt10\%Rh crucible.

The calcined melter feed was very hard and difficult to break even with a hammer. The entire batch was put back into the $4 \mathrm{~L}$ beaker used for drying and crushed to smaller size pieces which were then placed into an automated alumina mortar and pestle (also referred to as the alumina grinder), and crushed to a powder that was passed through a $425 \mu \mathrm{m}(40 \mathrm{mesh})$ stainless steel sieve to ensure homogeneity of the glass.

It had been determined that not enough of the AN-107 Sr/TRU precipitate had been added to the C-104 melter feed. The requisite amount of simulant Sr/TRU precipitate was dried, added to a portion of the melter feed, crushed in the alumina grinder, and sieved through the $425 \mu \mathrm{m}$ sieve. The melter feed was blended for about 5 minutes in a $250 \mathrm{~mL}$ glass jar by shaking, rotating, and turning the jar until the batch was well blended.

The high temperature furnace was heated to $1150^{\circ} \mathrm{C}$. Approximately one third of the batch was added to the Pt $10 \%$ Rh crucible (Figure 4.5 ) and placed in the furnace. Two more additions were made over time to complete the total addition of the melter feed into the crucible. Observations were made of the melt about every five minutes to check melt characteristics. Though foaming in the melt could not be observed, the foam lines on the crucible wall indicated that the batch volume doubled in size as the feed reacted. The small melter feed addition sizes kept the foam from reaching the top of the crucible. 


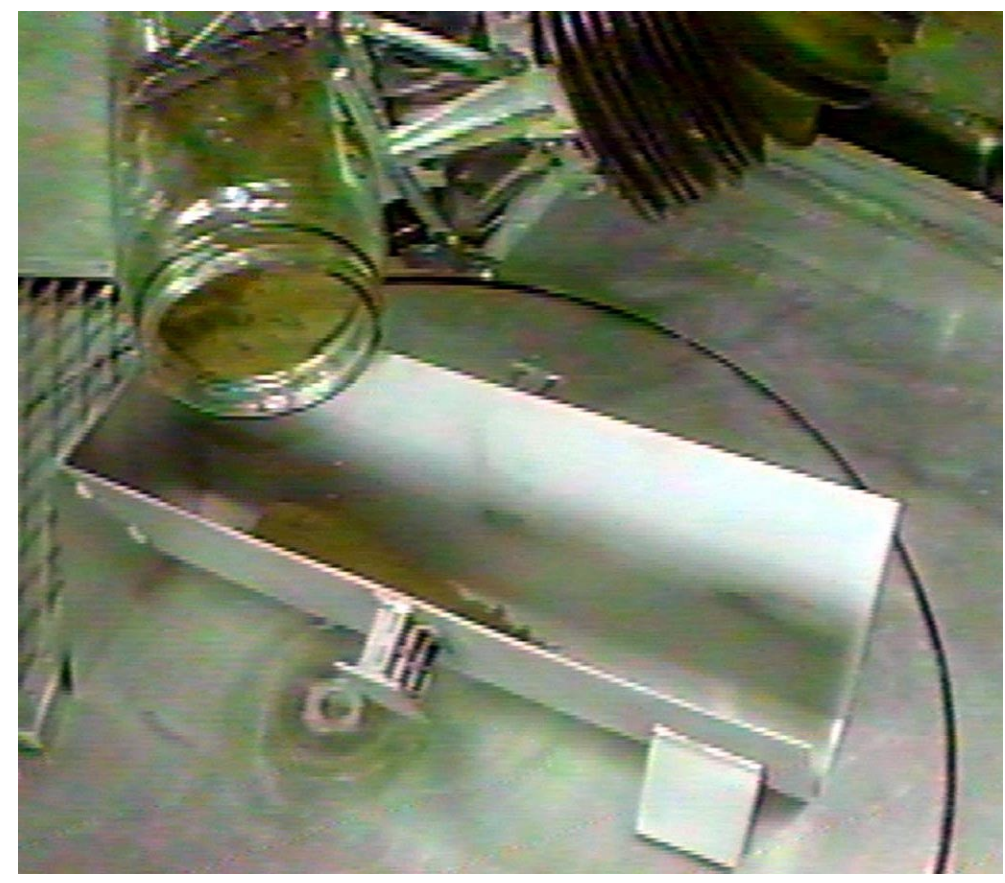

Figure 4.5. Powdered, calcined melter feed is added to a stainless steel funnel to help load it into the crucible in preparation for melting. The funnel is used to evenly distribute the powder into the hot crucible as additional batches of melter feed are added to the melt.

Melt observations were made as each feed addition to the crucibles were made. Foam had collapsed by the time the additions were made, but bubbles were present at the melt surface. The incorporation of solids into the liquid state was slow and undissolved feed was observed with each observation as scum or a dull, thin layer on the melt surface. After the final addition of feed, a Pt10\%Rh lid was place over the top of the crucible, and the melt temperature adjusted to $1150^{\circ} \mathrm{C}$.

After 1 hour the melt was again visually observed. The melt looked excellent. The glass surface was very smooth with no bubbles present or any signs of undissolved feed. The crucible was rotated from side to side to check viscosity and the melt moved easily with an estimated viscosity of $15 \mathrm{~Pa} \cdot \mathrm{s}$ based upon past experience. The crucible was placed back in the furnace with the lid and the melt continued for another hour.

The molten glass looked very good during the glass pour with no bubbles or undissolved feed observed. The glass was first poured into a $2.5 \mathrm{~cm}^{3}$ box shaped Pt $10 \% \mathrm{Rh}$ crucible (Figure 4.6) for canister centerline cooled (CCC) heat treatment of a portion of the glass. Immediately following, the remainder of the glass was air quenched on a stainless steel plate (Figure 4.6). The glass was covered with a stainless steel metal screen to prevent thermally stressed glass from flying out of the quench plate. Again, the viscosity of the molten glass while being poured was estimated to be $15 \mathrm{~Pa} \cdot \mathrm{s}$ based upon past experience. The resultant glass was stored in clean glass jars and segregated from glass that had contacted the crucible wall during the pour or had not been contained on the clean stainless steel surface while being prepared for storage. A total of $239.84 \mathrm{~g}$ of C-104 glass was produced, of which $37.36 \mathrm{~g}$ was poured into the box crucible for the $\mathrm{CCC}$ heat treatment. The box crucible was tightly fitted with a $\mathrm{Pt} 10 \% \mathrm{Rh}$ lid and stored until this heat treatment. 


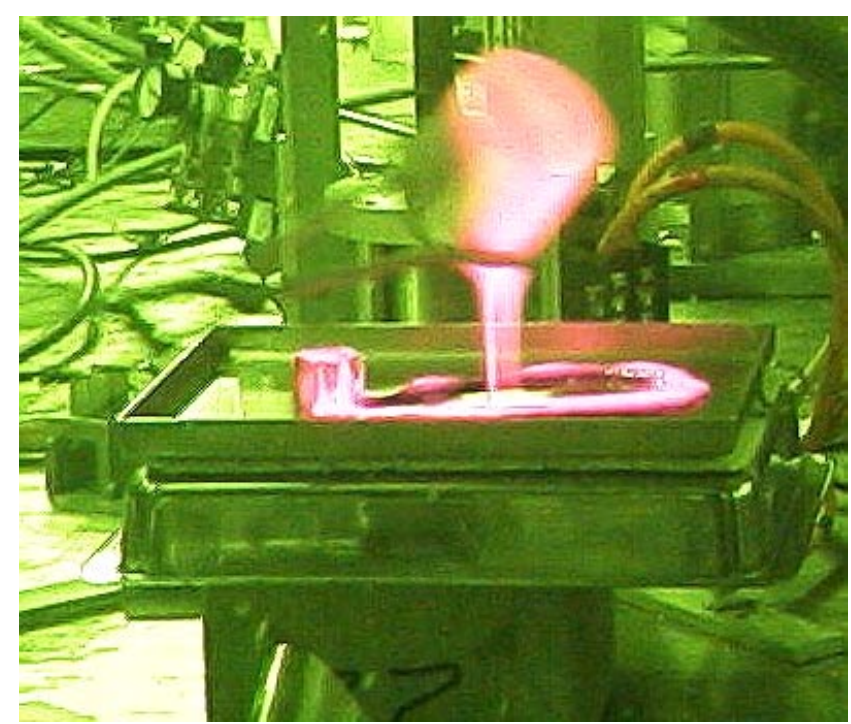

Figure 4.6. The C-104 glass sample is air quenched on a stainless steel tray. Note the box crucible full of molten glass on the left side of the glass pour.

Feed Preparation and Vitrification of the AZ-102 Glasses

It was necessary to complete both the physical and rheological properties tests and the vitrification and product testing scopes of work related to the pretreated AZ-102 tank sludge waste simultaneously; therefore, the pretreated AZ-102 waste was split into nearly equal amounts to make two AZ-102 glass melts. The first melt, AZ-102 Melt 1, did not have sugar added to the melter feed, i.e. did not simulate the actual BNFL HLW flowsheet, as it will not affect the crucible melt final glass product composition or properties being tested (PCT, crystalline and noncrystalline phase determination, chemical and radiochemical composition analysis, and regulatory analysis except for TCLP and SVOA). The second melt, AZ-102 Melt 2, was generated from the melter feed supplied by the physical and rheological properties testing task. This AZ-102 pretreated sludge and secondary waste split melter feed contained sugar in flowsheet amounts as sugar addition was necessary to accurately measure the melter feed flowsheet "Physical and Rheological Properties." Both AZ-102 Melt 1 and AZ-102 Melt 2 preparation and melt processes are explained separately below.

\section{Feed Preparation and Vitrification of the AZ-102 Melt 1 Glass (No Sugar Added)}

The pretreated AZ-102 tank sludge waste needed for processing AZ-102 Melt 1 (499.02 g) had been stored in a $2 \mathrm{~L}$ stainless steel beaker with a tightly fitted lid once the samples had been split: one part for this melt and the other portion for the physical and rheological properties testing. The beaker was transferred into hot cell "A" of the HLRF and placed on a stirrer/hot plate and heated to near boiling with the solution mixed using a rotating Teflon stir bar. Secondary wastes were added: $14.37 \mathrm{~g}$ of AN-107 simulant Sr/TRU precipitate, $69.38 \mathrm{~g}$ of low nitrate and $11.37 \mathrm{~g}$ of high nitrate Tc ion exchange eluant, and $157.29 \mathrm{~g}$ of the composite Cs ion exchange eluant solution.

The dry mineral additives, borax, $\left(\mathrm{Na}_{2} \mathrm{~B}_{4} \mathrm{O}_{7} \cdot 10 \mathrm{H}_{2} \mathrm{O}\right)$; sodium carbonate, $\left(\mathrm{Na}_{2} \mathrm{CO}_{3}\right)$; sodium metasilicate, $\left(\mathrm{Na}_{2} \mathrm{SiO}_{3} \cdot 5 \mathrm{H}_{2} \mathrm{O}\right)$; lithium hydroxide, $\left(\mathrm{LiOH} \cdot \mathrm{H}_{2} \mathrm{O}\right)$; and silica sand $\left(\mathrm{SiO}_{2}\right)$, were weighed, and blended together for 2 minutes in a $250 \mathrm{~mL}$ agate milling chamber in the Glass Development Laboratory located in the APEL facility. From a $116.93 \mathrm{~g}$ chemical batch, $111.36 \mathrm{~g}$ was weighed, transferred into hot 
cell "A", and the powder slowly poured into the heated waste slurry as it stirred. No visible changes were observed with the addition of the mineral additives, dissolution and suspension of the materials into the slurry went well.

Evaporation of most of the liquid took 5 hours, at which point the slurry was very thick (like molten fudge or chocolate) with a thin skin that developed on the surface and the slurry at the wall of the beaker 'jelled'. Manipulator stirring with the stainless steel stir rod was used and blended the total batch back into a smooth consistency. Three additional hours of stirring and heating made the slurry very thick and it was difficult to move the stir rod through the mixture, but the slurry maintained its smooth texture. The heat was turned off and the melter feed allowed to dry over night at $30 \pm 2{ }^{\circ} \mathrm{C}$, the hot cell ambient temperature.

The following morning the melter feed had dried and cracked similarly to dried mud. The melter feed was heated and began to soften from the heat forming into approximately 1 to $1.5 \mathrm{~cm}$ diameter chunks aided by mixing with the stir rod. Hot cell activities at the time (broken manipulator and scheduling constraints) would not allow the use of the furnaces, so the drying cycle was completed on the hot plate with a watch glass placed over the top of the beaker and a thermocouple resting in the middle of the melter feed. Over the next 3.5 hours, the sample was heated from about $100^{\circ}$ to $425^{\circ} \mathrm{C}$. The melter feed appeared dry and came easily out of the beaker and was added to the Pt10\%Rh crucible (Figure 4.7).

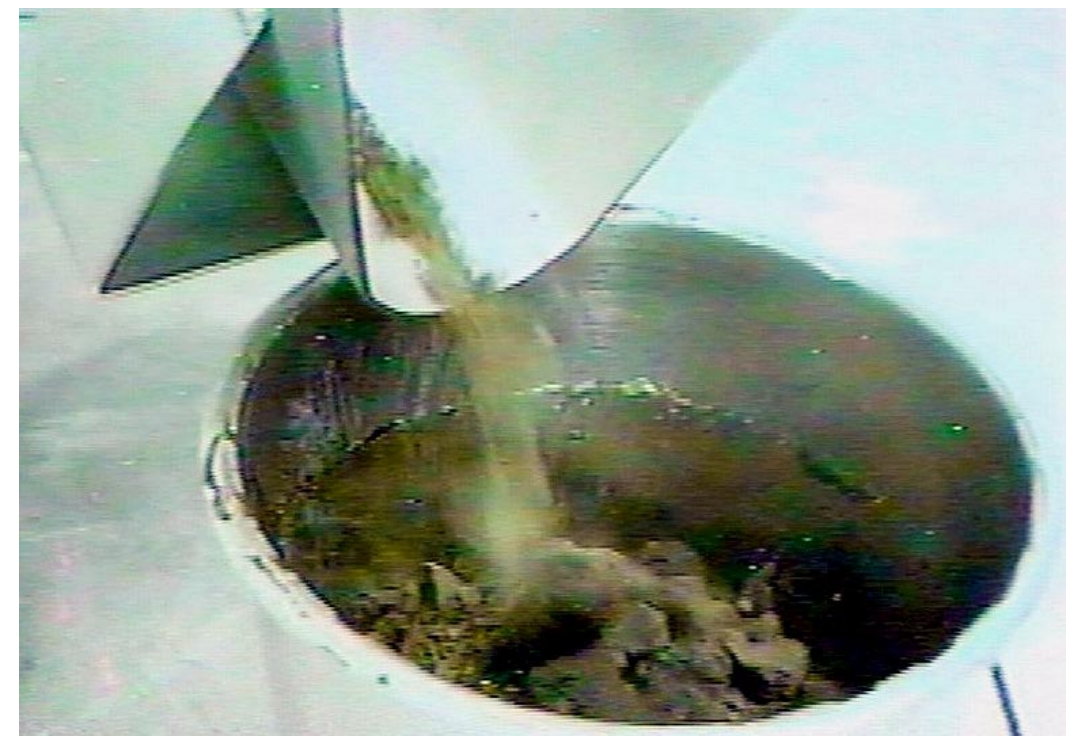

Figure 4.7. Dried AZ-102 Melt 1 melter feed is being loaded into the Pt10\%Rh crucible using the stainless steel funnel in preparation for calcining.

Calcining the sample had the same restraint as drying, so again the hot plate was used. The thermocouple was fitted into the dried melter feed just below its surface. A stainless steel beaker was fitted over the crucible to trap the heat and the temperature on the hot plate slowly increased until the dial reached "high". Temperature at the top of the calcining feed increased from 400 to $553^{\circ} \mathrm{C}$ over the first hour of heating and slowly rose to $559^{\circ} \mathrm{C}$ during the second hour. The melter feed sample was removed from the crucible and examined. The melter feed at the top of the crucible was still loose and light in color. At the crucible bottom, the melter feed batch changed to a dark gray and had sintered; the chunks of material were sticking together and to the crucible. The calcined feed was easily ground in the alumina grinder, sieved through the $425 \mu \mathrm{m}$ sieve, and mixed in a glass jar by rotating and tumbling the powdered sample. 
The high temperature furnace was heated to $1150^{\circ} \mathrm{C}$. About half of the batch was added to the Pt $10 \% \mathrm{Rh}$ crucible (Figure 4.8) and placed into the furnace. The final addition was made 7 minutes later after the melt quickly slumped without any foaming into a melt pool. After 14 minutes the melt looked good, the Pt $10 \% \mathrm{Rh}$ lid was added to the top of the crucible, and the melter feed sample was melted for two hours. Twenty five minutes before the melt pour, the temperature of the melt was raised to $1200^{\circ} \mathrm{C}$ to lower the glass melt viscosity, because of the small melt size (about $100 \mathrm{~g}$ ), to allow the glass melt to be more easily poured from the crucible.

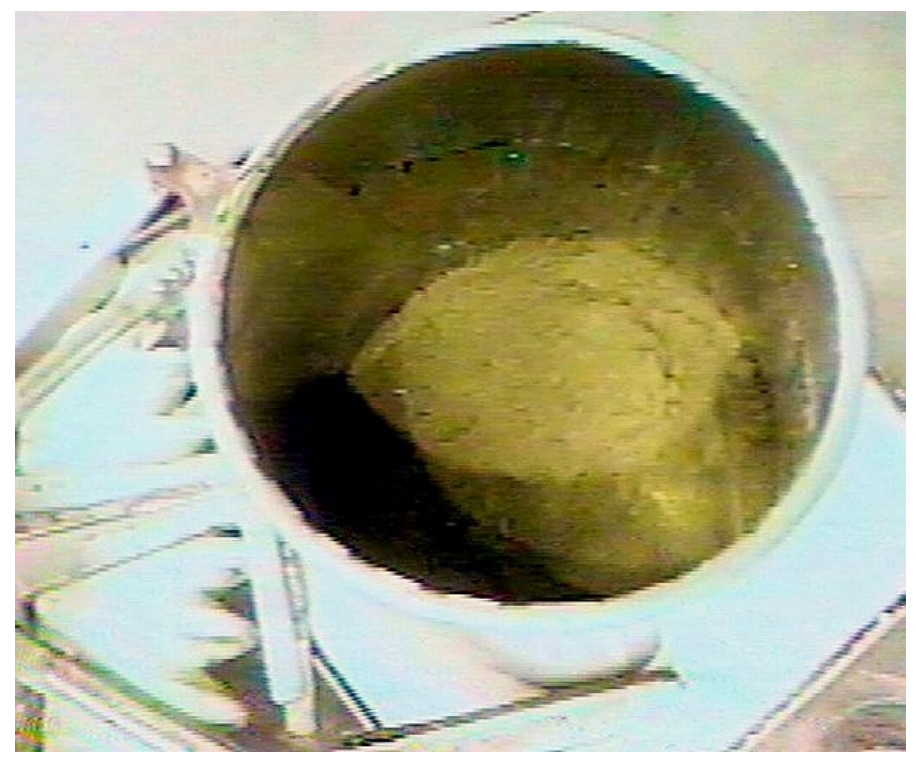

Figure 4.8. Approximately 50 percent of the AZ-102 Melt 1 powdered calcined feed loaded in the Pt10\%Rh crucible and ready for melting.

The molten glass looked very good during the glass pour with no bubbles or undissolved feed observed. The glass was very thick, estimated viscosity was $30 \mathrm{~Pa} \cdot \mathrm{s}$ based upon past experience. An attempt was made to pour the molten glass into a $2.5 \mathrm{~cm}^{3}$ box shaped Pt $10 \% \mathrm{Rh}$ crucible for canister centerline cooled (CCC) heat treatment, but only a small bead of glass covered the crucible bottom before the glass solidified. The poured glass was broken into 1 to $2 \mathrm{~cm}$ chunks, put into the box crucible, and weighed (29.07 g of glass). The box crucible was tightly fitted with a Pt10\%Rh lid and stored until the CCC heat treatment.

Following the initial glass pour of AZ-102 Melt 1, $86 \mathrm{~g}$ of glass remained after the CCC heat treatment sample had been secured. Of the remaining glass, 76.26 was ground and sieved through the 425 $\mu \mathrm{m}$ sieve in preparation for the glass to be added to AZ-102 Melt 2. However, it was decided that this glass would be used for the regulatory testing due to schedule constraints, so this glass powder was remelted to ensure it was suitable for testing. The glass powder was placed back in the Pt $10 \% \mathrm{Rh}$ crucible, put back into the high temperature furnace for 37 minutes at $1150^{\circ} \mathrm{C}$ then 28 minutes at $1216^{\circ} \mathrm{C}$, and poured onto the stainless steel plate. The glass pour was excellent with no bubbles present. The pour was smooth and flowed evenly at an estimated viscosity of $5 \mathrm{~Pa} \cdot \mathrm{s}$ based upon past experience. The glass was poured in a thin ribbon to accommodate preparation for the regulatory tests. Caution was taken to ensure the glass was handled and stored in a manner that kept the glass clean and free of organic contamination for the regulatory tests. 
Feed Preparation and Vitrification of the AZ-102 Melt 2 Glass (Sugar Added)

Safety concerns were raised about the AZ-102 Melt 2 melter feed_sugar-nitrate reaction (see Section 3.1.1, subsection "AZ-102 Melt 2 Melter Feed Batching" for more details) during the drying and calcining stages of the glass preparation process. To examine and determine the extent of the reaction for this particular melter feed being process in a confined space (the hot cells), a simulant melter feed was prepared, dried, calcined, and melted in the Glass Development Laboratory located in the APEL Building. The entire process went very smoothly with no observable signs of an excessive energetic exothermic reaction detected, even with a thermocouple monitoring the drying process.

The processing of AZ-102 Melt 2 was simple because the melter feed preparation was accomplished by the team of people that did the physical and rheological properties tests (Bredt et al., 2000). The AZ-102 Melt 2 melter feed came in a $250 \mathrm{~mL}$ glass jar with all melter feed components added. The feed was emptied into a $2 \mathrm{~L}$ stainless steel beaker and the jar rinsed clean (melter feed had a lot of solids on container bottom). The slurry was heated and mixed for 3.3 hours on a hot plate/stirrer until the slurry thickened enough that the Teflon stir bar could not rotate. The beaker was left open to the hot cell environment over the weekend to aid in drying. The melter feed was dried and cracked similarly to mud when examined the following Monday. The melter feed was dry enough to easily come out of the beaker and was added to the $300 \mathrm{~mL} \mathrm{Pt10 \% Rh} \mathrm{melting} \mathrm{crucible.}$

The melter feed was further dried on the hot plate from 120 to $400^{\circ} \mathrm{C}$ over several hours. The hot plate was used for both drying and calcining using a $2 \mathrm{~L}$ beaker as a cover along with the Pt $10 \% \mathrm{Rh}$ lid to trap the heat. A thermocouple was also placed at the top of the sample to monitor temperature. The sample was calcined for several hours at $600^{\circ} \mathrm{C}$. The entire drying/calcining process took 5 hours. The calcined material was ground in the alumina grinder, sieved through the $425 \mu \mathrm{m}$ sieve, and mixed in a glass jar.

The entire batch of melter feed was added to the Pt $10 \% \mathrm{Rh}$ crucible and placed in the high temperature furnace at $1150^{\circ} \mathrm{C}$ for a 2 hour melt after initially viewing the melter feed uneventfully melting and slumping into a molten pool. The lid covered the crucible during the $2 \mathrm{~h}$ melt and was removed at the end of the melt time, the temperature was then raised to $1200^{\circ} \mathrm{C}$ for 20 minutes, and the glass poured in a thin ribbon, (at an estimated viscosity of $5 \mathrm{~Pa} \cdot \mathrm{s}$ based upon past visual observations) onto the stainless steel plate (Figure 4.9). Caution was taken to ensure glass was handled and stored to keep it clean for TCLP, VOA and SVOA regulatory testing. 


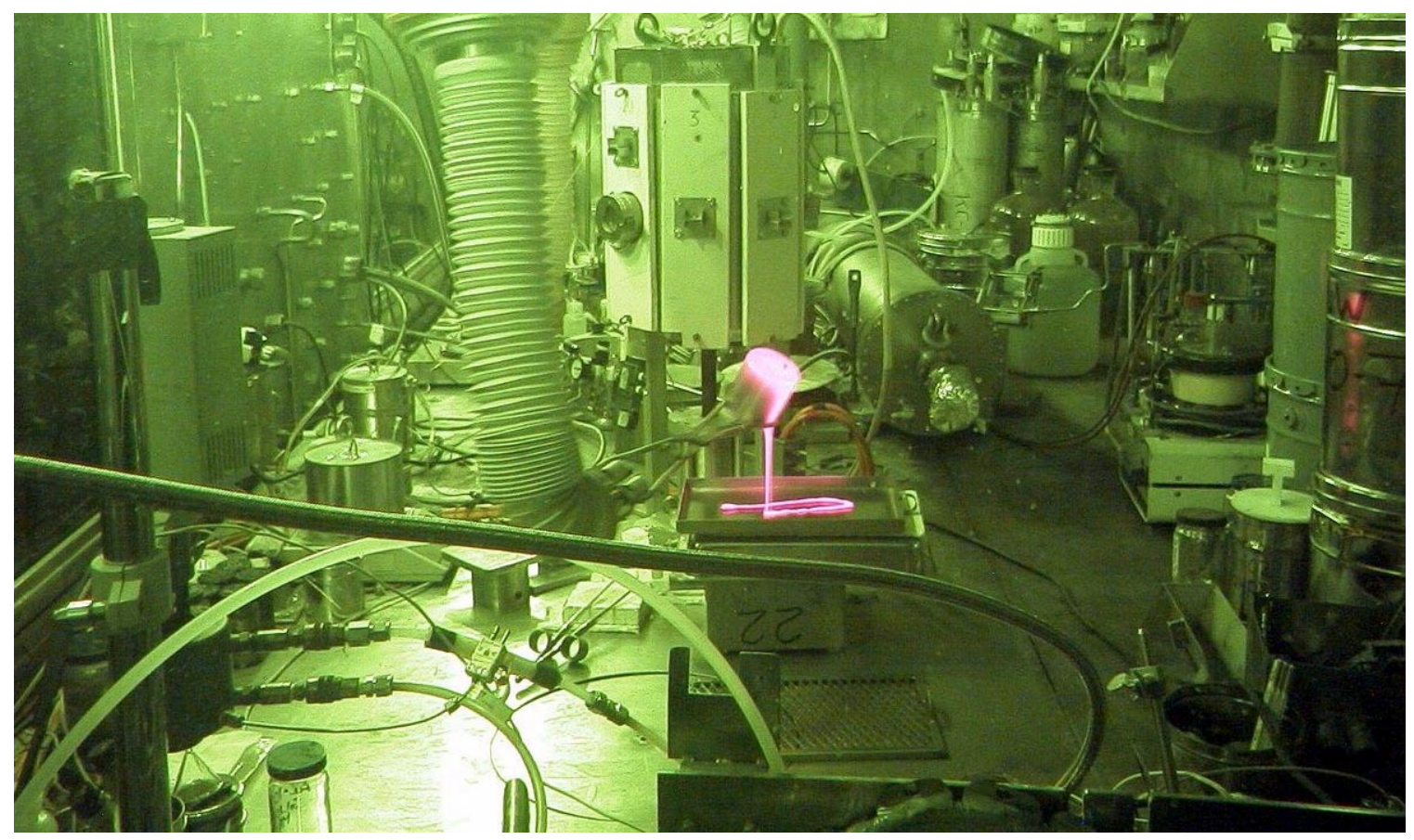

Figure 4.9. Molten AZ-102 Melt 2 glass being air quenched on a stainless steel plate.

\section{Feed Preparation and Vitrification of the HLW Process Blank Glass (AZ-102 Melt 1 Simulant)}

Feed preparation of the HLW Process Blank (AZ-102 Melt 1 simulant) was completed in the Glass Development Laboratory located in the APEL facility. Before melter feed preparation, the density, weight percent ( $\mathrm{wt} \%$ ) solids, and the total oxide per gram ( $\mathrm{g} \mathrm{TO} / \mathrm{g}$ ) of the WVDP simulant waste were measured and calculated to be $1.143 \mathrm{~g} / \mathrm{cm}^{3}, 13.33 \mathrm{wt} \%$, and $0.1119 \mathrm{~g} \mathrm{TO} / \mathrm{g}$, respectively. The WVDP simulant waste was weighed ( $446.8 \mathrm{~g}$, equal to $50 \mathrm{~g}$ of oxide) into a $2 \mathrm{~L}$ stainless steel beaker. The solution was heated and stirred on a hot plate/stirrer to evaporate the water. Chemical components were added to adjust the melter feed to near the composition of the AZ-102 Melt 1 glass. The simulant melter feed was dried on the hot plate, transferred to a drying oven and left at $107^{\circ} \mathrm{C}$ for nearly 5 days. The feed was then calcined for $2 \mathrm{~h}$ in a high temperature furnace at $600^{\circ} \mathrm{C}$. It was then ground to a fine powder using a tungsten carbide grinding chamber contained is a disc mill. The fine powder was put in a $250 \mathrm{~mL}$ plastic bottle and taken to the HLRF, hot cell "A".

The high temperature furnace was heated to $1150^{\circ} \mathrm{C}$. About half the batch was placed in the 250 $\mathrm{mL} \mathrm{Pt} 10 \% \mathrm{Rh}$ crucible, which filled it about one fourth full, and put into the furnace. Foaming of the melt half filled the crucible. After twenty minutes, the melt had settled into a molten pool; the remainder of the simulant melter feed batch was added to the melt. Thirteen minutes into the melt of the second chemical charge, vigorous foaming was observed above the top of the crucible. The foam was carefully monitored, but six minutes later it flowed over the top of the crucible and down the sides. The crucible was quickly removed, the foam cap pressed down into the crucible with tongs, and the melt cooled.

The foam on the outside of the crucible was chipped off with a hammer over the stainless steel pour plate to catch the broken pieces. When the crucible was clean, it was placed back into the furnace and melting resumed. Even after 15 minutes of melting, foam still persisted in the crucible though the volume of foam was much reduced. The chips of foam broken from the outside of the crucible were 
poured back into the crucible and melting resumed for an additional 15 minutes. Foam was still present in the crucible; the melt temperature was increased to $1200^{\circ} \mathrm{C}$. After 20 minutes of melting, foam still persisted so the temperature was raised to $1250^{\circ} \mathrm{C}$. The glass was removed 15 minutes later, observed, and poured. The melt had finally incorporated solids into it, the bubbles had finally burst, and the glass looked good. The melt poured at about $4 \mathrm{~Pa} \cdot \mathrm{s}$, based upon past visual observations, with no visible bubbles or volatile fumes (Figures 4.10 and 4.11). Caution was taken to ensure glass was handled and stored in a manner to keep the glass clean for regulatory tests.

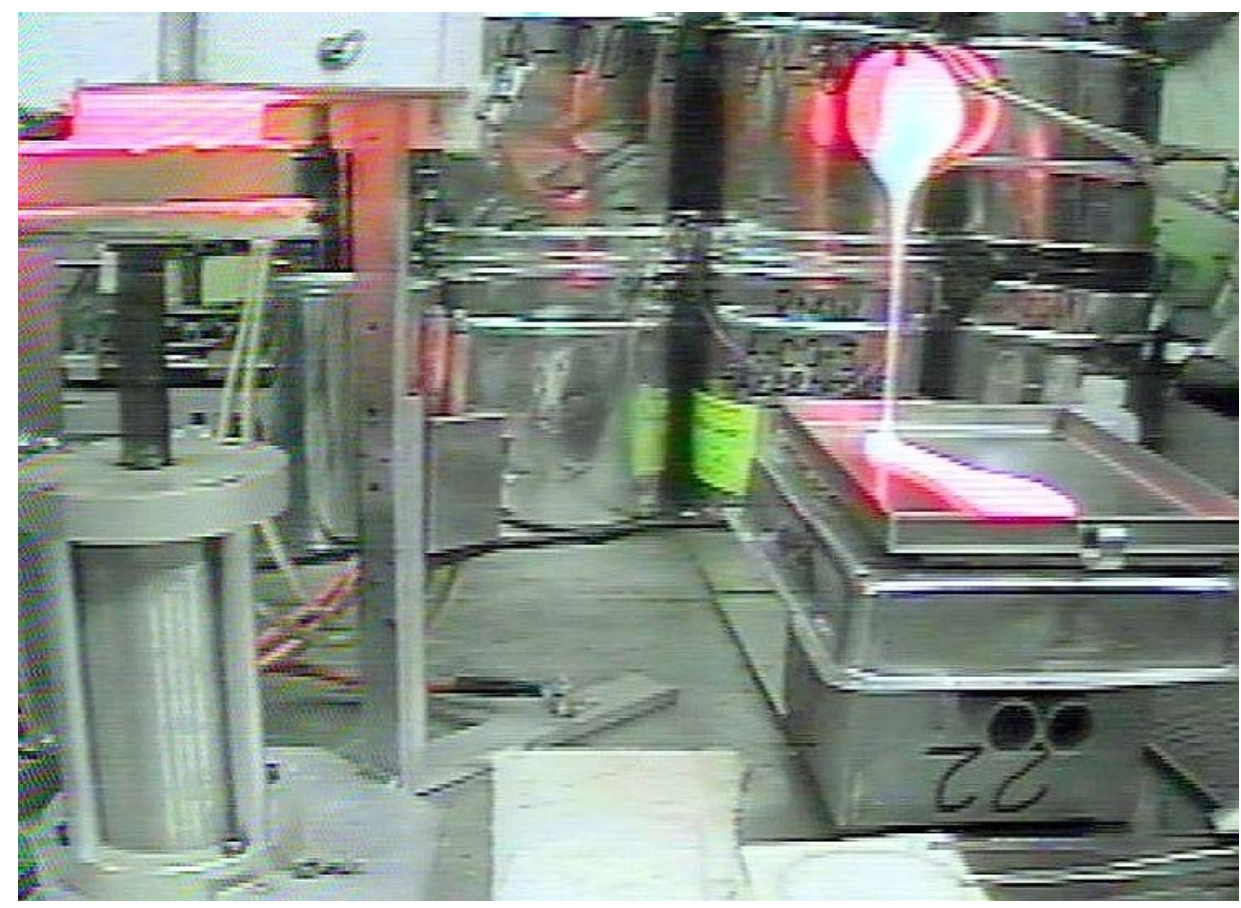

Figure 4.10. The HLW Process Blank (AZ-102 Melt 1 simulant) glass being poured.

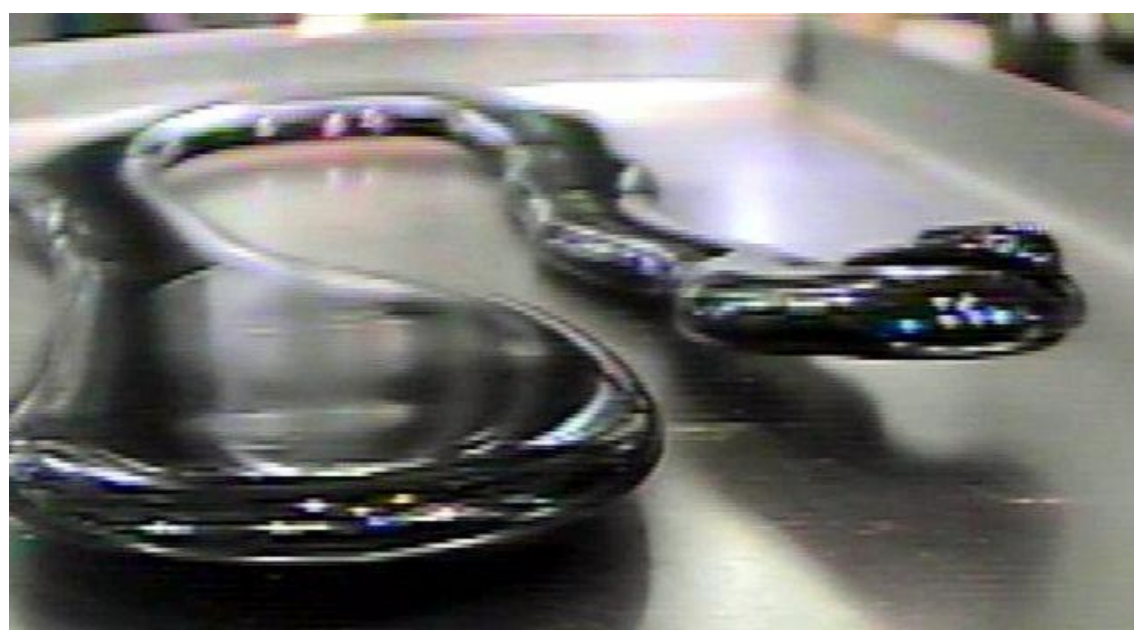

Figure 4.11. The HLW Process Blank (AZ-102 Melt 1 simulant) glass pour just after air quenching on the stainless steel tray. 


\subsubsection{Chemical Composition}

Chemical composition of the three HLW glasses (i.e., elements \{excluding oxygen\}) present in concentrations greater than 0.5 percent by weight) were measured in duplicate along with an ARG-1 powdered glass reference standard (Smith 1993) using a sodium peroxide $\left(\mathrm{Na}_{2} \mathrm{O}_{2}\right)$ fusion, according to procedure PNL-ALO-114, and a potassium hydroxide $(\mathrm{KOH})$ fusion, according to procedure PNL-ALO-115. Analytical Reference Glass-1 (ARG-1) is a compositionally well-characterized glass and provides an excellent independent check of the analytical processes and results. The $\mathrm{KOH}$ fusion uses a nickel crucible and the $\mathrm{Na}_{2} \mathrm{O}_{2}$ fusion uses a zirconium crucible. Cation analysis was performed using Inductively Coupled Plasma-Atomic Emission Spectrometry (ICP-AES).

\section{Statistically Based Renormalization of the ICP-AES Chemical Analyses Data}

$\mathrm{KOH}$ and $\mathrm{Na}_{2} \mathrm{O}_{2}$ fusion preparations and ICP-AES analyses were performed on each of the radioactive glasses, C-104 and AZ-102, as well as the glass reference standard, ARG-1 (Smith 1993). This process established elemental composition for contract compliance and allowed calculation of modified PCT normalized releases. Tables $4.1,4.2$, and 4.3 provide analyzed chemical compositions in $\mu \mathrm{g}$ element/gram glass and $\mathrm{wt} \%$ oxide. The reported $\mathrm{wt} \%$ oxide values are analytical 'process blank' corrected. The ARG-1 analysis is found in Appendix A, Table A.3. The table shows that the analytical $w t \%$ values agree with the target values for ARG-1 quite well indicating good analytical results.

Quality control objectives were met for all analytes whose concentration was equal to or greater than $0.5 \mathrm{wt} \%$ as required. Concentrations of analytes in the ARG-1 laboratory control standard (LCS) that were present at levels greater than the estimated quantification limits (EQL) were within $\pm 10 \%$ of the values listed for the "Consensus Composition Determined by Round Robin 6" (Table 3.1, Smith 1993). Except for zinc, all other analytes detected in the LCS were recovered within the acceptance limits of 75 to $125 \%$. Summation of measured wt $\%$ oxides in the LCS was about $99 \%$. The total accountability of mass in these glasses by ICP-AES ranges from 92.4 to $101.8 \%$ for the Envelope D glasses AZ-102 Melt 1 and Melt 2 and C-104. The discrepancy in total wt $\%$ oxides is because certain elements (such as $\mathrm{SO}_{3}$; the halides $\mathrm{Br}, \mathrm{Cl}$, and F; and trace metals) were not included in the analyses. Another reason is the possible incomplete recovery of $\mathrm{SiO}_{2}$ during the preparation of the sample for analysis and the difficulty in optimizing $\mathrm{Na}_{2} \mathrm{O}$ detection. As shown below when omitted or discrepant components are adjusted, the total wt $\%$ values for AZ-102 Melt 1 and Melt 2 and C-104 are quite close to $100 \mathrm{wt} \%$.

Tables 4.1, 4.2, and 4.3 list the target and measured wt\% oxide compositions for the AZ-102-Melt 1, AZ-102-Melt 2, and C-104 glasses. Measured compositions are listed separately for $\mathrm{K} / \mathrm{Ni}$ and $\mathrm{Na} / \mathrm{Zr}$ fusions of the glasses. It should be noted that the $\mathrm{Na}$ and $\mathrm{Zr}$ measurements in the $\mathrm{Na} / \mathrm{Zr}$ fusion are copied from the $\mathrm{K} / \mathrm{Ni}$ fusion measurements and the $\mathrm{K}$ and $\mathrm{Ni}$ measurements in the $\mathrm{K} / \mathrm{Ni}$ fusion are copied from the $\mathrm{Na} / \mathrm{Zr}$ fusion measurements. The remaining columns in Tables 4.1, 4.2, and 4.3 are discussed below.

Adjustments have been made to the measured data to generate as realistic as possible estimates of the composition of each of the three glasses due to potential analytical problems caused by: analytical detection limits greater than target values and possible analytical bias. The approach taken for undetected elements/oxides was to use their target values as the measurement instead of a blank measurement. Use of target values and bias correction for reporting of glass compositions are quite common as it is extremely expensive to analyze for the large number of cations, anions, etc. along with the large range of masses and isotopes contained in nuclear waste glass. Bias correction for nuclear waste glasses is therefore routinely done, e.g. see paper by Smith et al. 1997. The approach taken for bias correction was to analyze a well characterized glass at the same time as these three glasses to evaluate potential biases between measured $\mathrm{wt} \%$ oxides in a glass sample and the true $\mathrm{wt} \%$ oxides in the glass. Using nominal $\mathrm{wt} \%$ oxides and associated standard deviations for ARG-1 from the Materials Characterization Center 
(MCC) Round Robin (Smith 1993, PNL-8992), a 80\% prediction interval for a single observation was formed for each oxide as discussed in Hahn and Meeker (1991). An 80\% confidence level was used because the fact that ARG-1 was only analyzed once with AZ-102-Melt 1, AZ-102-Melt 2, and C-104 makes it statistically more difficult to declare significant biases when ARG-1 measured values differ from nominal values

The approach taken for potential analytical bias was to decide which bias corrections to use for the three glasses, if any. Candidates for bias correction met the following criteria:

- Detected in the ARG-1 glass and the other glass (necessary to allow bias calculation)

- The ratio of the nominal ARG-1 composition to the other glass target composition was within a reasonable range, selected as $1 / 5$ to 5 .

- The ARG-1 composition in the measured glass was outside of the $80 \%$ prediction interval based on the ARG-1 historical data.

Bias assessments were performed separately for the $\mathrm{K} / \mathrm{Ni}$ and $\mathrm{Na} / \mathrm{Zr}$ fusions. Based on the above criteria bias corrections were made for the following on all three glasses:

- $\mathrm{Na} / \mathrm{Zr}$ fusion: $\mathrm{CaO}, \mathrm{Na}_{2} \mathrm{O}$

- K/Ni fusion: $\mathrm{Al}_{2} \mathrm{O}_{3}, \mathrm{~B}_{2} \mathrm{O}_{3}, \mathrm{CaO}, \mathrm{Fe}_{2} \mathrm{O}_{3}, \mathrm{Li}_{2} \mathrm{O}, \mathrm{MnO}_{2}, \mathrm{Na}_{2} \mathrm{O}$, and $\mathrm{SiO}_{2}$

Tables 4.1, 4.2, and 4.3 also list the normalized adjusted compositions for the $\mathrm{Ni}$ and $\mathrm{Zr}$ fusions, and the averaged normalized adjusted composition (obtained by averaging the normalized adjusted compositions for the $\mathrm{K} / \mathrm{Ni}$ and $\mathrm{Na} / \mathrm{Zr}$ fusions). The total $\mathrm{wt} \%$ values for the adjusted $\mathrm{AZ}-102$ Melt 1 , AZ-102 Melt 2, and C-104 compositions are close enough to $100 \mathrm{wt} \%$ to renormalize the adjusted compositions so they total $100 \mathrm{wt} \%$. Renormalization of unadjusted measured compositions to $100 \mathrm{wt} \%$ can be inappropriate in that: (1) biases may not be properly addressed by the renormalization, and (2) renormalization to $100 \mathrm{wt} \%$ can induce biases in unbiased measured values. However, after appropriate bias corrections or adjustments, if total $\mathrm{wt} \%$ values are close enough to $100 \mathrm{wt} \%$ to suggest that all significant biases have likely been addressed, then renormalizing the adjusted compositions to $100 \mathrm{wt} \%$ is appropriate. In fact, it has been shown in the statistics literature that renormalization in such a case actually reduces the uncertainty in the estimated composition (Deming 1964).

The "Target" and "Averaged Normalized" composition columns in Tables 4.1, 4.2, and 4.3 agree quite well for oxides with higher target values with one apparent exception for AZ-102 Melt 1 and AZ-102 Melt 2. For those two glasses the averaged normalized adjusted values of $\mathrm{UO}_{2}$ are nearly twice their target values. Independent uranium isotopic analyses have been performed by ICP-MS and give values $1.72 \mathrm{wt} \% \mathrm{UO}_{2}$ for both AZ-102 Melt 1 and AZ-102 Melt 2 consistent with their target values of $1.63 \mathrm{wt} \%$ and $1.62 \mathrm{wt} \% \mathrm{UO}_{2}$. Note that the ICP-MS value for uranium for glass C-104 agrees with the analytical value. Those results indicate that the measured values for AZ-102 Melt 1 and AZ-102 Melt 2 are high by a factor of two. It is concluded that the target values are more accurate for uranium in AZ-102 Melt 1 and AZ-102 Melt 2.

Tables 4.1, 4.2, and 4.3 also list adjusted wt\% values for oxides in the AZ-102 Melt 1, AZ-102 Melt 2, and C-104 glasses. These adjusted values are either: (i) target (T) values for undetected or unanalyzed oxides, (ii) the measured (M) values (i.e., no adjustment), or (iii) bias corrected (BC) versions of measured values. One exception to use of a target value was for K in the AZ-102 Melt 2 glass. The target value in both AZ-102 glasses was approximately $0.025 \mathrm{wt} \%$. However, the analytical measurement for $\mathrm{K}$ in the AZ-102 Melt 2 glass was reported at the detection limit. Because of the relative insensitivity 
of the analytical method to $\mathrm{K}$, this resulted in an estimated $2.41 \mathrm{wt} \%$, nearly 100 times the target value. For this special case the measurement was replaced by the target value.

The "Target" and "Averaged Normalized" composition columns in Tables 4.1, 4.2, and 4.3 agree quite well for oxides with higher target values. The apparent problem with the analytical results for uranium in the glasses is believed to be a result of the very high detection limit ( $\sim 2.0 \mathrm{wt} \%)$ for this element under the conditions of the analysis (a dilution factor of $\sim 10000$ times). The uranium target value was less than the detection limit and the detected values were less than 1.5 times the detection limit, so there was a very large uncertainty attached to these analyses. This is all consistent with using the target values, which were calculated on the basis of the AZ-102 waste analysis, which is also consistent with ICP-MS results. 
Table 4.1. Target, Measured, Adjusted, and Normalized Adjusted Compositions of AZ-102 Melt 1 Radioactive Glass. See Table 4.4 for a compilation of the footnotes for Tables 4.1, 4.2, and 4.3

\begin{tabular}{|c|c|c|c|c|c|c|c|c|c|c|c|c|c|}
\hline \multirow{3}{*}{$\begin{array}{c}\text { Glass } \\
\text { Fusion } \\
\text { Oxide } \\
\end{array}$} & \multicolumn{3}{|c|}{ ARG - 1} & \multicolumn{10}{|c|}{ AZ-102 Melt 1} \\
\hline & \multirow{2}{*}{$\begin{array}{c}\text { Target } \\
\mathrm{wt} \%\end{array}$} & $\mathrm{Na} / \mathrm{Zr}$ & $\mathrm{K} / \mathrm{Ni}$ & \multirow{2}{*}{$\begin{array}{c}\text { Target } \\
\mathrm{wt} \% \\
\end{array}$} & \multicolumn{4}{|c|}{$\mathrm{Na} / \mathrm{Zr}^{(\mathrm{a})(\mathrm{b})(\mathrm{c})(\mathrm{d})}$} & \multicolumn{4}{|c|}{$\mathrm{K} / \mathrm{Ni}^{(\mathrm{a})(\mathrm{b})(\mathrm{c})(\mathrm{d})}$} & \multirow{2}{*}{$\begin{array}{c}\text { Average } \\
\text { Normalized }\end{array}$} \\
\hline & & \multicolumn{2}{|c|}{ Measured } & & Measured & Adjustment & Adjusted & Normalized & Measured & Adjustment & Adjusted & Normalized & \\
\hline $\mathrm{Ag}_{2} \mathrm{O}$ & & & & 0.0190 & & $\mathrm{~T}$ & 0.0190 & 0.0192 & 0.0349 & M & 0.0349 & 0.0352 & 0.0272 \\
\hline $\mathrm{Al}_{2} \mathrm{O}_{3}$ & 4.66 & 4.7792 & 4.1837 & 7.6880 & 8.1605 & M & 8.1605 & 8.2295 & 7.3199 & M & 7.3199 & 7.3781 & 7.8038 \\
\hline $\mathrm{B}_{2} \mathrm{O}_{3}$ & 8.54 & 8.5652 & 7.5679 & 3.9793 & 3.7030 & M & 3.7030 & 3.7343 & 3.3649 & $\mathrm{BC}$ & 3.7971 & 3.8273 & 3.7808 \\
\hline $\mathrm{BaO}$ & 0.09 & 0.0893 & 0.0766 & 0.0383 & 0.0446 & M & 0.0446 & 0.0450 & 0.0430 & $\mathrm{BC}$ & 0.0505 & 0.0509 & 0.0480 \\
\hline $\mathrm{BeO}$ & & & 0.0056 & 0.0026 & & $\mathrm{~T}$ & 0.0026 & 0 & & $\mathrm{~T}$ & 0 & 0 & 0 \\
\hline $\mathrm{CaO}$ & 1.42 & 1.5902 & 1.3153 & 0.4779 & 0.2168 & $\mathrm{BC}$ & 0.1936 & 0.1953 & & $\mathrm{~T}$ & 0.4779 & 0.4817 & 0.3385 \\
\hline $\mathrm{CdO}$ & & & & 1.3974 & 1.3076 & M & 1.3076 & 1.3187 & 1.2448 & M & 1.2448 & 1.2547 & 1.2867 \\
\hline $\mathrm{CeO}_{2}$ & & & & 0.0581 & & $\mathrm{~T}$ & 0.0581 & 0.0586 & & $\mathrm{~T}$ & 0.0581 & 0.0586 & 0.0586 \\
\hline $\mathrm{Co}_{2} \mathrm{O}_{3}$ & & & 0.0179 & 0.0409 & & $\mathrm{~T}$ & 0.0409 & 0.0412 & & $\mathrm{~T}$ & 0.0409 & 0.0412 & 0.0412 \\
\hline $\mathrm{Cr}_{2} \mathrm{O}_{3}$ & 0.10 & 0.1140 & 0.0906 & 0.0071 & 0.1147 & M & 0.1147 & 0.1157 & 0.1059 & M & 0.1059 & 0.1068 & 0.1112 \\
\hline $\mathrm{CuO}$ & 0.01 & & 0.0274 & 0.0922 & 0.0313 & M & 0.0313 & 0.0316 & 0.0651 & M & 0.0651 & 0.0656 & 0.0486 \\
\hline $\mathrm{Dy}_{2} \mathrm{O}_{3}$ & & & & 0.0296 & & $\mathrm{~T}$ & 0.0296 & 0.0299 & & $\mathrm{~T}$ & 0.0296 & 0.0298 & 0.0298 \\
\hline $\mathrm{Eu}_{2} \mathrm{O}_{3}$ & & & & 0.0092 & & $\mathrm{~T}$ & 0.0092 & 0.0093 & & $\mathrm{~T}$ & 0.0092 & 0.0093 & 0.0093 \\
\hline $\mathrm{Fe}_{2} \mathrm{O}_{3}$ & 14.02 & \begin{tabular}{l|l|}
2 & 14.1022
\end{tabular} & 12.1650 & 12.1492 & 12.1479 & M & 12.1479 & 12.2506 & 10.8037 & $\mathrm{BC}$ & 12.4511 & 12.5501 & 12.4004 \\
\hline $\mathrm{K}_{2} \mathrm{O}$ & 2.68 & 4.3380 & 4.3380 & 0.0258 & & $\mathrm{~T}$ & 0.0258 & 0.0260 & & $\mathrm{~T}$ & 0.0258 & 0.0260 & 0.0260 \\
\hline $\mathrm{La}_{2} \mathrm{O}_{3}$ & & & & 0.2983 & 0.2874 & M & 0.2874 & 0.2898 & 0.3108 & M & 0.3108 & 0.3133 & 0.3016 \\
\hline $\mathrm{Li}_{2} \mathrm{O}$ & 3.18 & 3.3156 & 2.6291 & 5.0000 & 4.9734 & M & 4.9734 & 5.0155 & 4.3921 & $\mathrm{BC}$ & 5.3125 & 5.3548 & 5.1851 \\
\hline $\mathrm{MgO}$ & 0.87 & 0.9451 & 0.7213 & 0.1198 & & $\mathrm{~T}$ & 0.1198 & 0.1208 & 0.2321 & M & 0.2321 & 0.2340 & 0.1774 \\
\hline $\mathrm{MnO}_{2}$ & 2.32 & 2.2954 & 2.0651 & 0.9225 & 1.1223 & M & 1.1223 & 1.1318 & 0.9672 & $\mathrm{BC}$ & 1.0866 & 1.0952 & 1.1135 \\
\hline $\mathrm{MoO}_{3}$ & & & & 0 & & $\mathrm{~T}$ & & 0 & & $\mathrm{~T}$ & 0 & 0 & 0 \\
\hline $\mathrm{Na}_{2} \mathrm{O}$ & 11.20 & 9.7137 & 9.7137 & 13.2719 & 11.7829 & $\mathrm{BC}$ & 13.5858 & 13.7007 & 11.7829 & $\mathrm{BC}$ & 13.5858 & 13.6938 & 13.6973 \\
\hline $\mathrm{Nd}_{2} \mathrm{O}_{3}$ & & & & 0.2100 & 0.2624 & M & 0.2624 & 0.2646 & 0.3381 & M & 0.3381 & 0.3408 & 0.3027 \\
\hline $\mathrm{NiO}$ & 1.04 & 1.0825 & 1.0800 & 0.7677 & 0.7803 & M & 0.7803 & 0.7869 & 0.7800 & M & 0.7800 & 0.7862 & 0.7866 \\
\hline $\mathrm{P}_{2} \mathrm{O}_{5}$ & 0.27 & 0.2980 & 0.2500 & 0.4624 & 0.5615 & M & 0.5615 & 0.5663 & 0.5730 & M & 0.5730 & 0.5776 & 0.5719 \\
\hline $\mathrm{PbO}$ & & & 0.0225 & 0.0932 & 0.0991 & M & 0.0991 & 0.0999 & 0.1992 & M & 0.1992 & 0.2008 & 0.1504 \\
\hline $\mathrm{PdO}$ & & & & 0 & & $\mathrm{~T}$ & & 0 & & $\mathrm{~T}$ & 0 & 0 & 0 \\
\hline $\mathrm{Rh}_{2} \mathrm{O}_{3}$ & & & & 0 & & $\mathrm{~T}$ & 0 & 0 & & $\mathrm{~T}$ & 0 & 0 & 0 \\
\hline $\mathrm{RuO}_{2}$ & & & & 0 & & $\mathrm{~T}$ & 0 & 0 & & $\mathrm{~T}$ & 0 & 0 & 0 \\
\hline $\mathrm{Sb}_{2} \mathrm{O}_{3}$ & & & & 0.0212 & & $\mathrm{~T}$ & 0.0212 & 0.0214 & & $\mathrm{~T}$ & 0.0212 & 0.0214 & 0.0214 \\
\hline $\mathrm{SiO}_{2}$ & 47.75 & $5 \quad 49.4340$ & 44.5393 & 47.9950 & 46.6520 & M & 46.6520 & 47.0466 & 43.1210 & $\mathrm{BC}$ & 46.2295 & 46.5971 & 46.8219 \\
\hline $\mathrm{SnO}_{2}$ & & & & 0.1638 & & $\mathrm{~T}$ & 0.1638 & 0.1652 & & $\mathrm{~T}$ & 0.1638 & 0.1651 & 0.1651 \\
\hline $\mathrm{SrO}$ & 0.005 & 0.0043 & 0.0040 & 1.4921 & 1.2154 & M & 1.2154 & 1.2257 & 1.2390 & M & 1.2390 & 1.2489 & 1.2373 \\
\hline $\mathrm{ThO}_{2}$ & & & & 0 & & $\mathrm{~T}$ & & 0 & & $\mathrm{~T}$ & 0 & 0 & 0 \\
\hline $\mathrm{TiO}_{2}$ & 1.17 & 1.1276 & 0.9361 & 0.0107 & & $\mathrm{~T}$ & 0.0107 & 0.0108 & & $\mathrm{~T}$ & 0.0107 & 0.0108 & 0.0108 \\
\hline $\mathrm{UO}_{2}$ & & & & 1.6230 & 2.4381 & $\mathrm{~T}$ & 1.6230 & 1.6367 & 3.7422 & $\mathrm{~T}$ & 1.6230 & 1.6359 & 1.6363 \\
\hline $\mathrm{V}_{2} \mathrm{O}_{3}$ & & & 0.0178 & 0.0036 & & $\mathrm{~T}$ & 0.0036 & 0.0036 & & $\mathrm{~T}$ & 0.0036 & 0.0036 & 0.0036 \\
\hline $\mathrm{Y}_{2} \mathrm{O}_{3}$ & & & & 0.0146 & & $\mathrm{~T}$ & 0.0146 & 0.0147 & & $\mathrm{~T}$ & 0.0146 & 0.0147 & 0.0147 \\
\hline $\mathrm{ZnO}$ & 0.02 & & 0.0224 & 0.0405 & & $\mathrm{~T}$ & 0.0405 & 0.0408 & & $\mathrm{~T}$ & 0.0405 & 0.0408 & 0.0408 \\
\hline $\mathrm{ZrO}_{2}$ & 0.14 & 0.1043 & 0.1040 & 1.4700 & 1.7360 & $\mathrm{M}$ & 1.7360 & 1.7507 & 1.7360 & $\mathrm{M}$ & 1.7360 & 1.7498 & 1.7503 \\
\hline Total & 99.485 & 101.8984 & 91.8932 & $99.995^{(\mathrm{f})}$ & 97.6372 & & 99.1612 & $100.0000^{(\mathrm{f})}$ & 92.3959 & & 99.2110 & $100.0000^{(\mathrm{f})}$ & $100.0000^{(\mathrm{f}}$ \\
\hline
\end{tabular}


Table 4.2. Target, Measured, Adjusted, and Normalized Adjusted Compositions of AZ-102 Melt 2 Radioactive Glass. See Table 4.4 for a compilation of the footnotes for Tables 4.1, 4.2, and 4.3

\begin{tabular}{|c|c|c|c|c|c|c|c|c|c|c|c|c|c|}
\hline Glass & & ARG-1 & & & & & & AZ-102 & Melt 2 & & & & \\
\hline Fusion & Target & $\mathrm{Na} / \mathrm{Zr}$ & $\mathrm{K} / \mathrm{Ni}$ & Target & & $\mathrm{Na} / \mathrm{Zr}^{(\mathrm{a})}$ & (b) (c) (d) & & & $\mathrm{K} / \mathrm{Ni}^{(\mathrm{a})}$ & (b) (c) (d) & & Average \\
\hline Oxide & $\mathrm{wt} \%$ & Meast & ured & wt $\%$ & Measured & Adjustment & Adjusted & Normalized & Measured & Adjustment & Adjusted & Normalized & Normalized \\
\hline $\mathrm{Ag}_{2} \mathrm{O}$ & & & & 0.0194 & & $\mathrm{~T}$ & 0.0194 & 0.0190 & 0.0311 & M & 0.0311 & 0.0293 & 0.0242 \\
\hline $\mathrm{Al}_{2} \mathrm{O}_{3}$ & 4.66 & 4.7792 & 4.1837 & 7.6942 & 7.9149 & M & 7.9149 & 7.7719 & 7.3577 & $\mathrm{BC}$ & 8.1952 & 7.7047 & 7.7383 \\
\hline $\mathrm{B}_{2} \mathrm{O}_{3}$ & 8.54 & 8.5652 & 7.5679 & 3.9993 & 3.8962 & M & 3.8962 & 3.8258 & 3.6225 & $\mathrm{BC}$ & 4.0878 & 3.8431 & 3.8345 \\
\hline $\mathrm{BaO}$ & 0.09 & 0.0893 & 0.0766 & 0.0392 & 0.0446 & M & 0.0446 & 0.0438 & 0.0424 & M & 0.0424 & 0.0399 & 0.0419 \\
\hline $\mathrm{BeO}$ & & & 0.0056 & 0.0026 & & $\mathrm{~T}$ & 0.0026 & 0.0026 & & $\mathrm{~T}$ & 0.0026 & 0.0024 & 0.0025 \\
\hline $\mathrm{CaO}$ & 1.42 & 1.5902 & 1.3153 & 0.4939 & 0.2658 & $\mathrm{BC}$ & 0.2374 & 0.2331 & 0.4337 & $\mathrm{BC}$ & 0.4682 & 0.4402 & 0.3366 \\
\hline $\mathrm{CdO}$ & & & & 1.3863 & 1.3247 & M & 1.3247 & 1.3008 & 1.2562 & M & 1.2562 & 1.1810 & 1.2409 \\
\hline $\mathrm{CeO}_{2}$ & & & & 0.0623 & & $\mathrm{~T}$ & 0.0623 & 0.0612 & & $\mathrm{~T}$ & 0.0623 & 0.0586 & 0.0599 \\
\hline $\mathrm{Co}_{2} \mathrm{O}_{3}$ & & & 0.0179 & 0.0407 & & $\mathrm{~T}$ & 0.0407 & 0.0400 & & $\mathrm{~T}$ & 0.0407 & 0.0383 & 0.0391 \\
\hline $\mathrm{Cr}_{2} \mathrm{O}_{3}$ & 0.10 & 0.1140 & 0.0906 & 0.0071 & 0.1308 & M & 0.1308 & 0.1284 & 0.1315 & M & 0.1315 & 0.1236 & 0.1260 \\
\hline $\mathrm{CuO}$ & 0.01 & & 0.0274 & 0.1107 & 0.0438 & M & 0.0438 & 0.0430 & 0.0814 & M & 0.0814 & 0.0765 & 0.0598 \\
\hline $\mathrm{Dy}_{2} \mathrm{O}_{3}$ & & & & 0.0294 & & $\mathrm{~T}$ & 0.0294 & 0.0289 & & $\mathrm{~T}$ & 0.0294 & 0.0276 & 0.0283 \\
\hline $\mathrm{Eu}_{2} \mathrm{O}_{3}$ & & & & 0.0092 & & $\mathrm{~T}$ & 0.0092 & 0.0090 & & $\mathrm{~T}$ & 0.0092 & 0.0086 & 0.0088 \\
\hline $\mathrm{Fe}_{2} \mathrm{O}_{3}$ & 14.02 & 14.1022 & 12.1650 & 12.2394 & 12.1836 & M & 12.1836 & 11.9635 & 11.3542 & $\mathrm{BC}$ & 13.0856 & 12.3024 & 12.1329 \\
\hline $\mathrm{K}_{2} \mathrm{O}$ & 2.68 & 4.3380 & 4.3380 & 0.0253 & 2.4100 & $\mathrm{~T}$ & 0.0253 & 0.0248 & 2.4100 & $\mathrm{~T}$ & 0.0253 & 0.0238 & 0.0243 \\
\hline $\mathrm{La}_{2} \mathrm{O}_{3}$ & & & & 0.2996 & 0.2874 & M & 0.2874 & 0.2822 & 0.3108 & M & 0.3108 & 0.2922 & 0.2872 \\
\hline $\mathrm{Li}_{2} \mathrm{O}$ & 3.18 & 3.3156 & 2.6291 & 5.0000 & 5.2641 & M & 5.2641 & 5.1690 & 4.7797 & $\mathrm{BC}$ & 5.7813 & 5.4352 & 5.3021 \\
\hline $\mathrm{MgO}$ & 0.87 & 0.9451 & 0.7213 & 0.1203 & & $\mathrm{~T}$ & 0.1203 & 0.1181 & 0.2155 & M & 0.2155 & 0.2026 & 0.1604 \\
\hline $\mathrm{MnO}_{2}$ & 2.32 & 2.2954 & 2.0651 & 1.0275 & 1.3804 & M & 1.3804 & 1.3554 & 1.3004 & $\mathrm{BC}$ & 1.4609 & 1.3735 & 1.3645 \\
\hline $\mathrm{MoO}_{3}$ & & & & 0.0 & & $\mathrm{~T}$ & 0 & 0 & & $\mathrm{~T}$ & 0 & 0 & 0 \\
\hline $\mathrm{Na}_{2} \mathrm{O}$ & 11.20 & 9.7137 & 9.7137 & 13.9099 & 12.3288 & $\mathrm{BC}$ & 14.2153 & 13.9585 & 12.3288 & $\mathrm{BC}$ & 14.2153 & 13.3644 & 13.6614 \\
\hline $\mathrm{Nd}_{2} \mathrm{O}_{3}$ & & & & 0.2185 & 0.2565 & $\mathrm{M}$ & 0.2565 & 0.2519 & 0.3148 & M & 0.3148 & 0.2960 & 0.2739 \\
\hline $\mathrm{NiO}$ & 1.04 & 1.0825 & 1.0800 & 0.7619 & 0.7707 & M & 0.7707 & 0.7568 & 0.7700 & M & 0.7700 & 0.7239 & 0.7404 \\
\hline $\mathrm{P}_{2} \mathrm{O}_{5}$ & 0.27 & 0.2980 & 0.2500 & 0.4613 & 0.4813 & M & 0.4813 & 0.4726 & 0.5157 & M & 0.5157 & 0.4848 & 0.4787 \\
\hline $\mathrm{PbO}$ & & & 0.0225 & 0.1121 & 0.1508 & M & 0.1508 & 0.1481 & 0.2046 & M & 0.2046 & 0.1924 & 0.1702 \\
\hline $\mathrm{PdO}$ & & & & 0.0052 & & $\mathrm{~T}$ & 0.0052 & 0.0051 & & $\mathrm{~T}$ & 0.0052 & 0.0049 & 0.0050 \\
\hline $\mathrm{Rh}_{2} \mathrm{O}_{3}$ & & & & 0.0000 & & $\mathrm{~T}$ & 0 & 0 & & $\mathrm{~T}$ & 0 & 0 & 0 \\
\hline $\mathrm{RuO}_{2}$ & & & & 0.0000 & & $\mathrm{~T}$ & 0 & 0 & & $\mathrm{~T}$ & 0 & 0 & 0 \\
\hline $\mathrm{Sb}_{2} \mathrm{O}_{3}$ & & & & 0.0210 & & $\mathrm{~T}$ & 0.0210 & 0.0206 & & $\mathrm{~T}$ & 0.0210 & 0.0197 & 0.0202 \\
\hline $\mathrm{SiO}_{2}$ & 47.75 & 49.4340 & 44.5393 & 47.0773 & 46.4380 & M & 46.4380 & 45.5991 & 44.9400 & $\mathrm{BC}$ & 48.1796 & 45.2958 & 45.4474 \\
\hline $\mathrm{SnO}_{2}$ & & & & 0.1625 & & $\mathrm{~T}$ & 0.1625 & 0.1596 & & $\mathrm{~T}$ & 0.1625 & 0.1528 & 0.1562 \\
\hline $\mathrm{SrO}$ & 0.005 & 0.0043 & 0.0040 & 1.4986 & 1.4868 & M & 1.4868 & 1.4599 & 1.5694 & M & 1.5694 & 1.4755 & 1.4677 \\
\hline $\mathrm{ThO}_{2}$ & & & & 0.0060 & & $\mathrm{~T}$ & 0.0060 & 0.0059 & & $\mathrm{~T}$ & 0.0060 & 0.0056 & 0.0058 \\
\hline $\mathrm{TiO}_{2}$ & 1.17 & 1.1276 & 0.9361 & 0.0109 & & $\mathrm{~T}$ & 0.0109 & 0.0107 & 0.0404 & M & 0.0404 & 0.0379 & 0.0243 \\
\hline $\mathrm{UO}_{2}$ & & & & 1.6191 & 2.9484 & M & 2.9484 & 2.8951 & 3.1752 & M & 3.1752 & 2.9851 & 2.9401 \\
\hline $\mathrm{V}_{2} \mathrm{O}_{3}$ & & & 0.0178 & 0.0036 & & $\mathrm{~T}$ & 0.0036 & 0.0035 & & $\mathrm{~T}$ & 0.0036 & 0.0034 & 0.0035 \\
\hline $\mathrm{Y}_{2} \mathrm{O}_{3}$ & & & & 0.0144 & & $\mathrm{~T}$ & 0.0144 & 0.0141 & & $\mathrm{~T}$ & 0.0144 & 0.0135 & 0.0138 \\
\hline $\mathrm{ZnO}$ & 0.02 & & 0.0224 & 0.0410 & & $\mathrm{~T}$ & 0.0410 & 0.0403 & & $\mathrm{~T}$ & 0.0410 & 0.0385 & 0.0394 \\
\hline $\mathrm{ZrO}_{2}$ & 0.14 & 0.1043 & 0.1040 & 1.4702 & 1.8103 & M & 1.8103 & 1.7776 & 1.8103 & $\mathrm{M}$ & 1.8103 & 1.7020 & 1.7398 \\
\hline Total & 99.485 & 101.8984 & 91.8932 & $100.000^{(\mathrm{f})}$ & 101.8180 & & 101.8398 & $100.0000^{(\mathrm{f})}$ & 98.9964 & & 106.3666 & $100.0000^{(\mathrm{f})}$ & $100.0000^{(\mathrm{f}}$ \\
\hline
\end{tabular}


Table 4.3. Target, Measured, Adjusted, and Normalized Adjusted Compositions of C-104 Radioactive Glass. See Table 4.4 for a compilation of the footnotes for Tables 4.1, 4.2, and 4.3

\begin{tabular}{|c|c|c|c|c|c|c|c|c|c|c|c|c|c|}
\hline \multirow{3}{*}{$\begin{array}{l}\text { Glass } \\
\text { Fusion } \\
\text { Oxide }\end{array}$} & \multicolumn{3}{|c|}{ ARG-1 } & \multicolumn{10}{|c|}{ C-104 } \\
\hline & \multirow{2}{*}{$\begin{array}{c}\text { Target } \\
\mathrm{wt} \%\end{array}$} & $\mathrm{Na} / \mathrm{Zr}$ & $\mathrm{K} / \mathrm{Ni}$ & \multirow{2}{*}{$\begin{array}{c}\text { Target } \\
\mathrm{wt} \%\end{array}$} & \multicolumn{4}{|c|}{$\mathrm{Na} / \mathrm{Zr}^{\text {(a) (b) (c) (d) }}$} & \multicolumn{4}{|c|}{$\mathrm{K} / \mathrm{Ni}^{(\mathrm{a})(\mathrm{b})(\mathrm{c})(\mathrm{d})}$} & \multirow{2}{*}{$\begin{array}{c}\text { Average } \\
\text { Normalized }\end{array}$} \\
\hline & & \multicolumn{2}{|c|}{ Measured } & & Measured & Adjustment & Adjusted & Normalized & Measured & Adjustment & Adjusted & Normalized & \\
\hline $\mathrm{Ag}_{2} \mathrm{O}$ & & & & 0.0657 & 0.0333 & M & 0.0333 & 0.0327 & 0.0585 & M & 0.0585 & 0.0580 & 0.0453 \\
\hline $\mathrm{Al}_{2} \mathrm{O}_{3}$ & 4.66 & 4.7792 & 4.1837 & 2.3585 & 2.6257 & M & 2.6257 & 2.5791 & 2.4463 & $\mathrm{BC}$ & 2.7247 & 2.6988 & 2.6389 \\
\hline $\mathrm{B}_{2} \mathrm{O}_{3}$ & 8.54 & 8.5652 & 7.5679 & 9.0081 & 9.1448 & M & 9.1448 & 8.9823 & 8.4203 & $\mathrm{BC}$ & 9.5019 & 9.4116 & 9.1970 \\
\hline $\mathrm{BaO}$ & 0.09 & 0.0893 & 0.0766 & 0.0191 & 0.0246 & M & 0.0246 & 0.0241 & 0.0234 & M & 0.0234 & 0.0232 & 0.0237 \\
\hline $\mathrm{BeO}$ & & & 0.0056 & 0.0051 & & $\mathrm{~T}$ & 0.0051 & 0.0050 & & $\mathrm{~T}$ & 0.0051 & 0.0051 & 0.0050 \\
\hline $\mathrm{CaO}$ & 1.42 & 1.5902 & 1.3153 & 0.4565 & 1.0143 & $\mathrm{BC}$ & 0.9057 & 0.8896 & 0.4267 & $\mathrm{BC}$ & 0.4607 & 0.4563 & 0.6729 \\
\hline $\mathrm{CdO}$ & & & & 0.0611 & 0.0582 & M & 0.0582 & 0.0572 & 0.0554 & M & 0.0554 & 0.0549 & 0.0560 \\
\hline $\mathrm{CeO}_{2}$ & & & & 0.0810 & & $\mathrm{~T}$ & 0.0810 & 0.0796 & & $\mathrm{~T}$ & 0.0810 & 0.0802 & 0.0799 \\
\hline $\mathrm{Co}_{2} \mathrm{O}_{3}$ & & & 0.0179 & 0.0023 & & $\mathrm{~T}$ & 0.0023 & 0.0023 & & $\mathrm{~T}$ & 0.0023 & 0.0023 & 0.0023 \\
\hline $\mathrm{Cr}_{2} \mathrm{O}_{3}$ & 0.10 & 0.1140 & 0.0906 & 0.1385 & 0.1534 & M & 0.1534 & 0.1507 & 0.1388 & M & 0.1388 & 0.1375 & 0.1441 \\
\hline $\mathrm{CuO}$ & 0.01 & & 0.0274 & 0.0256 & & $\mathrm{~T}$ & 0.0256 & 0.0251 & 0.0351 & M & 0.0351 & 0.0347 & 0.0299 \\
\hline $\mathrm{Dy}_{2} \mathrm{O}_{3}$ & & & & & & $\mathrm{~T}$ & 0 & 0 & & $\mathrm{~T}$ & 0 & 0 & 0 \\
\hline $\mathrm{Eu}_{2} \mathrm{O}_{3}$ & & & & & & $\mathrm{~T}$ & 0 & 0 & & $\mathrm{~T}$ & 0 & 0 & 0 \\
\hline $\mathrm{Fe}_{2} \mathrm{O}_{3}$ & 14.02 & 14.1022 & 12.1650 & 4.7179 & 4.4330 & M & 4.4330 & 4.3543 & 4.1327 & $\mathrm{BC}$ & 4.7629 & 4.7176 & 4.5359 \\
\hline $\mathrm{K}_{2} \mathrm{O}$ & 2.68 & \begin{tabular}{|l|l|} 
& 4.3380
\end{tabular} & 4.3380 & 0.0606 & & $\mathrm{~T}$ & 0.0606 & 0.0595 & & $\mathrm{~T}$ & 0.0606 & 0.0600 & 0.0598 \\
\hline $\mathrm{La}_{2} \mathrm{O}_{3}$ & & & & 0.0190 & & $\mathrm{~T}$ & 0.0190 & 0.0187 & & $\mathrm{~T}$ & 0.0190 & 0.0188 & 0.0187 \\
\hline $\mathrm{Li}_{2} \mathrm{O}$ & 3.18 & 3.3156 & 2.6291 & 5.0045 & 5.4256 & M & 5.4256 & 5.3292 & 4.9734 & $\mathrm{BC}$ & 6.0157 & 5.9585 & 5.6438 \\
\hline $\mathrm{MgO}$ & 0.87 & 0.9451 & 0.7213 & 0.0592 & & $\mathrm{~T}$ & 0.0592 & 0.0581 & & $\mathrm{~T}$ & 0.0592 & 0.0586 & 0.0584 \\
\hline $\mathrm{MnO}_{2}$ & 2.32 & 2.2954 & 2.0651 & 2.5486 & 3.0077 & M & 3.0077 & 2.9543 & 2.7228 & $\mathrm{BC}$ & 3.0588 & 3.0297 & 2.9920 \\
\hline $\mathrm{MoO}_{3}$ & & & & 0.0020 & & $\mathrm{~T}$ & 0.0020 & 0.0020 & & $\mathrm{~T}$ & 0.0020 & 0.0020 & 0.0020 \\
\hline $\mathrm{Na}_{2} \mathrm{O}$ & 11.20 & 9.7137 & 9.7137 & 8.5711 & 7.9613 & $\mathrm{BC}$ & 9.1795 & 9.0164 & 7.9613 & $\mathrm{BC}$ & 9.1795 & 9.0922 & 9.0543 \\
\hline $\mathrm{Nd}_{2} \mathrm{O}_{3}$ & & & & 0.0446 & & $\mathrm{~T}$ & 0.0446 & 0.0438 & & $\mathrm{~T}$ & 0.0446 & 0.0442 & 0.0440 \\
\hline $\mathrm{NiO}$ & 1.04 & 1.0825 & 1.0800 & 0.2365 & 0.2423 & M & 0.2423 & 0.2379 & 0.2104 & M & 0.2400 & 0.2377 & 0.2378 \\
\hline $\mathrm{P}_{2} \mathrm{O}_{5}$ & 0.27 & 0.2980 & 0.2500 & 0.3301 & 0.3438 & M & 0.3438 & 0.3377 & 0.4011 & M & 0.4011 & 0.3973 & 0.3675 \\
\hline $\mathrm{PbO}$ & & & 0.0225 & 0.1536 & 0.2046 & M & 0.2046 & 0.2010 & 0.1992 & M & 0.1992 & 0.1974 & 0.1992 \\
\hline $\mathrm{PdO}$ & & & & 0.0109 & & $\mathrm{~T}$ & 0.0109 & 0.0107 & & $\mathrm{~T}$ & 0.0109 & 0.0108 & 0.0108 \\
\hline $\mathrm{Rh}_{2} \mathrm{O}_{3}$ & & & & 0.0323 & & $\mathrm{~T}$ & 0.0323 & 0.0317 & & $\mathrm{~T}$ & 0.0323 & 0.0320 & 0.0319 \\
\hline $\mathrm{RuO}_{2}$ & & & & 0.0164 & & $\mathrm{~T}$ & 0.0164 & 0.0161 & & $\mathrm{~T}$ & 0.0164 & 0.0162 & 0.0162 \\
\hline $\mathrm{Sb}_{2} \mathrm{O}_{3}$ & & & & & & $\mathrm{~T}$ & 0 & 0 & & $\mathrm{~T}$ & 0 & 0 & 0 \\
\hline $\mathrm{SiO}_{2}$ & 47.75 & 49.4340 & 44.5393 & 47.8667 & 48.1500 & $\mathrm{M}$ & 48.1500 & 47.2947 & 43.8700 & $\mathrm{BC}$ & 47.0325 & 46.5854 & 46.9400 \\
\hline $\mathrm{SnO}_{2}$ & & & & 0.0687 & & $\mathrm{~T}$ & 0.0687 & 0.0675 & & $\mathrm{~T}$ & 0.0687 & 0.0680 & 0.0678 \\
\hline $\mathrm{SrO}$ & 0.005 & 0.0043 & 0.0040 & 3.3905 & 2.9618 & M & 2.9618 & 2.9092 & 2.9677 & M & 2.9677 & 2.9395 & 2.9243 \\
\hline $\mathrm{ThO}_{2}$ & & & & 4.1008 & 4.1537 & M & 4.1537 & 4.0799 & 3.5278 & M & 3.5278 & 3.4943 & 3.7871 \\
\hline $\mathrm{TiO}_{2}$ & 1.17 & 1.1276 & 0.9361 & 0.0166 & 0.0450 & M & 0.0450 & 0.0442 & & $\mathrm{~T}$ & 0.0166 & 0.0164 & 0.0303 \\
\hline $\mathrm{UO}_{2}$ & & & & 3.6353 & 3.9123 & M & 3.9123 & 3.8428 & 3.9123 & M & 3.9123 & 3.8751 & 3.8590 \\
\hline $\mathrm{V}_{2} \mathrm{O}_{3}$ & & & 0.0178 & 0.0038 & & $\mathrm{~T}$ & 0.0038 & 0.0037 & & $\mathrm{~T}$ & 0.0038 & 0.0038 & 0.0037 \\
\hline $\mathrm{Y}_{2} \mathrm{O}_{3}$ & & & & 0.0030 & & $\mathrm{~T}$ & 0.0030 & 0.0029 & & $\mathrm{~T}$ & 0.0030 & 0.0030 & 0.0030 \\
\hline $\mathrm{ZnO}$ & 0.02 & & 0.0224 & 2.0017 & 2.0729 & M & 2.0729 & 2.0361 & 1.9422 & $\mathrm{M}$ & 1.9422 & 1.9237 & 1.9799 \\
\hline $\mathrm{ZrO}_{2}$ & 0.14 & 0.1043 & 0.1040 & 4.8440 & 4.2962 & $\mathrm{M}$ & 4.2962 & 4.2199 & 4.2962 & $\mathrm{M}$ & 4.2962 & 4.2553 & 4.2376 \\
\hline Total & 99.485 & 101.8984 & 91.8932 & $99.960^{(\mathrm{f})}$ & 100.2644 & & 101.8085 & $100.0000^{(\mathrm{f})}$ & 92.7215 & & $\mid$\begin{tabular}{||c}
$\mid 00.9598$ \\
\end{tabular} & $100.0000^{(\mathrm{f})}$ & $100.0000^{(}$ \\
\hline
\end{tabular}


Table 4.4. Compilation of the Footnotes for Tables 4.1, 4.2, and 4.3

\section{Footnotes for Tables 4.1, 4.2, and 4.3}

(a) See text for description of how adjusted values were determined.

(b) Adjustment types: $\mathrm{T}=$ target, $\mathrm{M}=$ measured, $\mathrm{BC}=$ bias corrected. Target values were used when no values were measured or when measured values were less than detection limits. Relative bias corrections were applied based on ARG-1, for those oxides having statistically significant biases on ARG-1 measurements.

(c) Adjusted values normalized to total $100 \mathrm{wt} \%$.

(d) Average of normalized Ni fusion and $\mathrm{Zr}$ fusion compositions.

(e) Set to target value as best available estimate of actual value.

(f) Total is prior to rounding entries to four decimal places.

\section{HLW Glasses C-104, AZ-102 Melt 1, and AZ-102 Melt 2 Composition}

One of the objectives for the glass product waste loading for the HLW glasses was to meet the requirements delineated in the RPP-WTP Phase B-1 contract, specification 1.2.2.1.6, titled Product Loading, which states: "Loading of non-volatile components in Envelope D, and, if directed by DOE, entrained solids after washing in accordance with Specification 12, Number of HLW Canister Per Batch of Waste Envelope D, shall be achieved, such that, the concentration of at least one of the waste components or waste component combinations in Table TS-1.1 Minimum Component Limits in HLW Glass exceeds its minimum weight percent in HLW glass as identified in Table TS-1.1 ..." For the C-104, AZ-102 Melt 1, and AZ-102 Melt 2 HLW glasses the following is true:

- C-104: The total of all waste oxides (exclusive of Si) not identified in Table TS-1.1 is greater than $8.0 \%$.

- AZ-102 Melt 1: $\mathrm{Al}_{2} \mathrm{O}_{3}+\mathrm{Fe}_{2} \mathrm{O}_{3}+\mathrm{ZrO}_{2}$ is greater than $21.0 \%$.

- AZ-102 Melt 2: $\mathrm{Al}_{2} \mathrm{O}_{3}+\mathrm{Fe}_{2} \mathrm{O}_{3}+\mathrm{ZrO}_{2}$ is greater than $21.0 \%$.

The total of all waste oxides (exclusive of $\mathrm{Si}$ ) not identified in Table TS-1.1 was calculated for the C-104 glass, taking into account the glass former minerals added (see Table 3.6) using the "Average Normalized" weight percent oxide values listed in Table 4.3. The total waste percent oxide in the C-104 glass $\left(\mathrm{B}_{2} \mathrm{O}_{3}\right.$ of $0.020 ; \mathrm{BeO}$ of $0.005 ; \mathrm{CeO}_{2}$ of $0.079 ; \mathrm{Co}_{2} \mathrm{O}_{3}$ of $0.002 ; \mathrm{CuO}$ of $0.030 ; \mathrm{La}_{2} \mathrm{O}_{3}$ of $0.019 ; \mathrm{MnO}$ of 2.992; $\mathrm{MoO}_{3}$ of $0.002 ; \mathrm{Nd}_{2} \mathrm{O}_{3}$ of $0.044 ; \mathrm{SnO}_{2}$ of $0.068 ; \mathrm{SrO}$ of $2.924 ; \mathrm{ThO}_{2}$ of $3.787 ; \mathrm{V}_{2} \mathrm{O}_{3}$ of 0.004 ; $\mathrm{Y}_{2} \mathrm{O}_{3}$ of 0.003 ; and $\mathrm{ZnO}$ of 0.036 ) comes to $10.02 \%$ which is greater than the required $8.0 \%$. The total of $\mathrm{Al}_{2} \mathrm{O}_{3}+\mathrm{Fe}_{2} \mathrm{O}_{3}+\mathrm{ZrO}_{2}$ for AZ-102 Melt 1 is $21.96 \%$ and for AZ-102 Melt 2 is $21.61 \%$ as indicated in Table 4.6 and Table 4.7.

In Tables 4.5, 4.6, and 4.7 the waste or additive loading fraction in the glass is calculated from the dilution (decrease in concentration) of the element oxide concentrations contained either in the waste or in the glass forming additives in the final glass. The calculation is particularly simple when the diluted element oxide is contained in only one of the two components. For this calculation, the concentration of the element oxide in the glass is divided by the concentration of the element oxide in either the waste component or the additive component. For the C-104 glass, the boron oxide level in the glass was measured as $9.197 \mathrm{wt} \%$ and its concentration as part of the additives was $13.44 \mathrm{wt} \%$ and their ratio is 0.6843. Again for C-104, using an element oxide contributed only by the waste such as iron oxide, the ratio is found to be 0.3183 . Table 4.5 summarizes these calculations for the $\mathrm{C}-104$ glass. Note that the dilution factors for the additive dilution and the waste dilution theoretically will add up to 1.00 .

Therefore, the waste loading also can be calculated by subtracting the average additive dilution fraction from 1.00 (i.e., $1.0000-0.6895=0.3105$ ). The average factors and their sum, based on the measured 
oxide values for the glass is $0.3359+0.6895=1.0254$. The results indicate that the waste fraction for the $\mathrm{C}-104$ glass is very close to the target value of $33.1 \%$.

Table 4.5. Waste Loading/Dilution factors for HLW Waste Glass C-104

\begin{tabular}{|c|c|c|c|c|c|}
\hline \multicolumn{6}{|c|}{ C-104 } \\
\hline Oxide & Waste & Additives & Glass & Waste Dilution & Additive Dilution \\
\hline & wt\% oxide & wt\% oxide & $\begin{array}{l}\text { Normalized } \\
\text { wt } \% \text { oxide }\end{array}$ & (Glass/ Waste) & (Glass/Additive) \\
\hline $\mathrm{Al}_{2} \mathrm{O}_{3}$ & 7.13 & & 2.639 & 0.3701 & \\
\hline $\mathrm{B}_{2} \mathrm{O}_{3}$ & & 13.44 & 9.197 & & 0.6843 \\
\hline $\mathrm{Cr}_{2} \mathrm{O}_{3}$ & 0.42 & & 0.1441 & 0.3430 & \\
\hline $\mathrm{Fe}_{2} \mathrm{O}_{3}$ & 14.25 & & 4.536 & 0.3183 & \\
\hline $\mathrm{Li}_{2} \mathrm{O}$ & 0.10 & 7.4319 & 5.644 & & $0.7549 *$ \\
\hline $\mathrm{MnO}$ & 7.70 & & 2.992 & 0.3886 & \\
\hline $\mathrm{SiO}_{2}$ & 4.81 & 69.17 & 46.94 & & $0.6566^{*}$ \\
\hline $\mathrm{ThO}_{2}$ & 12.39 & & 3.79 & 0.3059 & \\
\hline $\mathrm{ZnO}$ & 0.11 & 2.9363 & 1.980 & & $0.6620 *$ \\
\hline $\mathrm{ZrO}_{2}$ & 14.63 & & 4.24 & 0.2898 & \\
\hline \multicolumn{6}{|c|}{ * Value takes into account the amount of that oxide in the waste oxides. } \\
\hline \multicolumn{4}{|c|}{ Average Dilution of Waste and Additive Components } & $\begin{array}{l}\text { 0.3359 } \\
\text { (Target 0.331) }\end{array}$ & $\begin{array}{l}0.6895 \\
\text { (Target 0.669) }\end{array}$ \\
\hline
\end{tabular}

In Table 4.6 the waste or additive loading fraction in the glass AZ-102 Melt 1 was calculated from the dilution (decrease in concentration) of the element oxide concentrations contained either in the waste or in the glass forming additives in the final glass as it was for C-104 above. Therefore, the waste loading also can be calculated by subtracting the average additive dilution fraction from 1.00 (i.e., 1.0000 $-0.6585=0.3415)$. The average factors and their sum, based on the measured oxide values for the glass is $0.3314+0.6584=0.9898$. The results indicate that the waste fraction for the AZ-102 Melt 1 glass is close to the target value of $33.39 \%$.

In Table 4.7 the waste or additive loading fraction in the glass AZ-102 Melt 2 was calculated from the dilution (decrease in concentration) of the element oxide concentrations contained either in the waste or in the glass forming additives in the final glass as it was for C-104, and AZ-102 Melt 1 above. Therefore, the waste loading also can be calculated by subtracting the average additive dilution fraction from 1.00 (i.e., $1.0000-0.6578=0.3422$ ). The average factors and their sum, based on the measured oxide values for the glass is $0.3398+0.6578=0.9976$. The results indicate that the waste fraction for the AZ-102 Melt 2 glass is close to the target value of $33.97 \%$.

Summarizing, the waste component concentration factors are consistent with the requirements delineated in the RPP-WTP contract, specification 1.2.2.1.6. Also, the waste loading was calculated from the dilution factor (decrease in concentration) of elements contained in either the waste or the glass forming additives. The results indicate that the waste fraction of each glass is near their target, i.e. $33.1 \%$ for C-104 (measured 33.59\% based on waste dilution and 31.05\% based on additive dilution), 33.39\% for AZ-102 Melt 1 (measured 33.14\% based on waste dilution and $34.16 \%$ based on additive dilution), and $33.97 \%$ for AZ-102 Melt 2 (measured 33.98\% based on waste dilution and $34.22 \%$ based on additive dilution). The measured glass to target composition percent difference comparison of the oxides is small and the calculated waste loading values are very close to or exceed the target. Both support the conclusion that the actual waste loading in each glass met or exceeded the target waste loading. 
Table 4.6. Waste Loading/Dilution factors for HLW Waste Glass AZ-102 Melt 1

\begin{tabular}{|c|c|c|c|c|c|}
\hline \multicolumn{6}{|c|}{ AZ-102 Melt 1} \\
\hline \multirow[t]{2}{*}{ Oxide } & $\begin{array}{l}\text { Processed } \\
\text { Waste }\end{array}$ & Additives & Glass & Waste Dilution & Additive Dilution \\
\hline & wt\% oxide & wt\% oxide & $\begin{array}{c}\text { Normalized } \\
\text { wt\% oxide }\end{array}$ & (Glass/ Waste) & (Glass/Additive) \\
\hline $\mathrm{Al}_{2} \mathrm{O}_{3}$ & 23.02 & & 7.804 & 0.3390 & \\
\hline $\mathrm{B}_{2} \mathrm{O}_{3}$ & & 5.95 & 3.781 & & 0.6355 \\
\hline $\mathrm{CaO}$ & 1.43 & & 0.3385 & 0.2367 & \\
\hline $\mathrm{CdO}$ & 4.19 & & 1.287 & 0.3072 & \\
\hline $\mathrm{Fe}_{2} \mathrm{O}_{3}$ & 36.39 & & 12.40 & 0.3408 & \\
\hline $\mathrm{La}_{2} \mathrm{O}_{3}$ & 0.89 & & 0.3016 & 0.3389 & \\
\hline $\mathrm{Li}_{2} \mathrm{O}$ & & 7.51 & 5.185 & & 0.6904 \\
\hline $\mathrm{MnO}$ & 2.76 & & 1.114 & 0.4036 & \\
\hline $\mathrm{NiO}$ & 2.30 & & 0.7866 & 0.3420 & \\
\hline $\mathrm{SiO}_{2}$ & 1.92 & 71.10 & 46.82 & & $0.6495 *$ \\
\hline $\mathrm{SrO}$ & 4.47 & & 1.237 & 0.2767 & \\
\hline $\mathrm{ZrO}_{2}$ & 4.40 & & 1.750 & 0.3977 & \\
\hline \multicolumn{6}{|c|}{ * Value takes into account the amount of that oxide in the waste oxides. } \\
\hline \multicolumn{4}{|c|}{ Average Dilution of Waste and Additive Components } & $\begin{array}{c}\mathbf{0 . 3 3 1 4} \\
\text { (Target } 0.3339) \\
\end{array}$ & $\begin{array}{c}0.6584 \\
\text { (Target } 0.6661 \text { ) } \\
\end{array}$ \\
\hline
\end{tabular}

Table 4.7. Waste Loading/Dilution factors for HLW Waste Glass AZ-102 Melt 2

\begin{tabular}{|c|c|c|c|c|c|}
\hline \multicolumn{6}{|c|}{ AZ-102 Melt 2} \\
\hline \multirow[t]{2}{*}{ Oxide } & $\begin{array}{l}\text { Processed } \\
\text { Waste }\end{array}$ & Additives & Glass & Waste Dilution & Additive Dilution \\
\hline & wt\% oxide & wt\% oxide & $\begin{array}{l}\text { Normalized } \\
\text { wt\% oxide }\end{array}$ & (Glass/ Waste) & (Glass/Additive) \\
\hline $\mathrm{Al}_{2} \mathrm{O}_{3}$ & 22.65 & & 7.738 & 0.3416 & \\
\hline $\mathrm{B}_{2} \mathrm{O}_{3}$ & & 6.03 & 3.835 & & 0.6360 \\
\hline $\mathrm{CaO}$ & 1.45 & & 0.337 & 0.2324 & \\
\hline $\mathrm{CdO}$ & 4.08 & & 1.241 & 0.3042 & \\
\hline $\mathrm{Fe}_{2} \mathrm{O}_{3}$ & 36.03 & & 12.13 & 0.3367 & \\
\hline $\mathrm{La}_{2} \mathrm{O}_{3}$ & 0.88 & & 0.2872 & 0.3264 & \\
\hline $\mathrm{Li}_{2} \mathrm{O}$ & & 7.57 & 5.302 & & 0.7004 \\
\hline $\mathrm{MnO}$ & 3.02 & & 1.365 & 0.4520 & \\
\hline $\mathrm{NiO}$ & 2.24 & & 0.7404 & 0.3305 & \\
\hline $\mathrm{SiO}_{2}$ & 1.99 & 70.27 & 45.45 & & $0.6372 *$ \\
\hline $\mathrm{SrO}$ & 4.41 & & 1.468 & 0.3329 & \\
\hline $\mathrm{ZrO}_{2}$ & 4.33 & & 1.740 & 0.4018 & \\
\hline \multicolumn{6}{|c|}{ Value takes into account the amount of that oxide in the waste oxides. } \\
\hline \multicolumn{4}{|c|}{$\begin{array}{c}\text { Average Dilution of Waste and Additive } \\
\text { Components }\end{array}$} & $\begin{array}{c}\mathbf{0 . 3 3 9 8} \\
\text { (Target } 0.3397)\end{array}$ & $\begin{array}{c}0.6578 \\
\text { (Target } 0.6603 \text { ) }\end{array}$ \\
\hline
\end{tabular}




\subsubsection{Radiochemical Composition}

Radiochemical analyses were performed on two of the IHLW glass products, i.e. C-104 and AZ-102 Melt1 glasses. Analyses included ${ }^{60} \mathrm{Co},{ }^{106} \mathrm{Ru},{ }^{125} \mathrm{Sb},{ }^{134} \mathrm{Cs},{ }^{137} \mathrm{Cs},{ }^{144} \mathrm{Ce},{ }^{154} \mathrm{Eu},{ }^{155} \mathrm{Eu}$, and ${ }^{241} \mathrm{Am}$ by GEA, ${ }^{90} \mathrm{Sr},{ }^{239 / 240} \mathrm{Pu},{ }^{241} \mathrm{Pu}$, and total uranium. Concentration values of additional gamma emitters (i.e., ${ }^{51} \mathrm{Cr},{ }^{59} \mathrm{Fe},{ }^{79} \mathrm{Se},{ }^{95} \mathrm{Nb},{ }^{103} \mathrm{Ru},{ }^{113} \mathrm{Sn}$, and ${ }^{152} \mathrm{Eu}$ ) were obtained by GEA depending on concentrations and detection limits. The reported errors $(1-\sigma)$ in Appendix $C$ represent the total propagated error including counting, dilution, yield, and calibration errors, as appropriate. Laboratory and process blank values (Appendix C) given with each analysis are the best indicators of the method detection limits, taking into account the actual sample sizes and counting times used for each analysis.

Samples of waste glass, C-104 and AZ-102 Melt 1, were initially processed in the shielded analytical laboratory (SAL). A nominal $0.1 \mathrm{~g}$ sample was fused using $\mathrm{Na}_{2} \mathrm{O}_{2}$ flux according to PNLALO-114, and brought to a $100-\mathrm{mL}$ volume. The samples were prepared in duplicate with a process blank. Aliquots of these solutions were distributed to the radiochemistry laboratory for subsequent radiochemical analyses.

Gamma Spectrometry. Quantifiable concentrations of ${ }^{60} \mathrm{Co},{ }^{134} \mathrm{Cs},{ }^{137} \mathrm{Cs},{ }^{154} \mathrm{Eu},{ }^{155} \mathrm{Eu}$, and ${ }^{241} \mathrm{Am}$ were measured in all samples. The AZ-102 Melt 1 samples also contained ${ }^{125} \mathrm{Sb}$. No other gammaemitting analytes were found; analysis for ${ }^{106} \mathrm{Ru}$ and ${ }^{144} \mathrm{Ce}$ was requested and their MDAs are provided (see Appendix C). The SAL process blank contained ${ }^{134} \mathrm{Cs},{ }^{137} \mathrm{Cs}$, and ${ }^{241} \mathrm{Am}$ concentrations at least three orders of magnitude lower than those found in the samples. The C-104 sample and duplicate analyte concentrations were within 17\% RPD. The AZ-102 Melt 1 sample and duplicate analyte concentrations were within $8 \%$ RPD.

The additional list of gamma emitting isotopes $\left({ }^{51} \mathrm{Cr},{ }^{59} \mathrm{Fe},{ }^{95} \mathrm{Nb},{ }^{103} \mathrm{Ru},{ }^{113} \mathrm{Sn}\right.$, and $\left.{ }^{152} \mathrm{Eu}\right)$ was evaluated. All isotope concentrations were below the method detection limits that are listed in the data table. The detection limits for the $\mathrm{C}-104$ samples in $\mu \mathrm{Ci} / \mathrm{g}$ are: ${ }^{51} \mathrm{Cr}<5 . \mathrm{E}+0,{ }^{59} \mathrm{Fe}<1 . \mathrm{E}-1,{ }^{95} \mathrm{Nb}<6 . \mathrm{E}-2$, ${ }^{103} \mathrm{Ru}<4 . \mathrm{E}+0,{ }^{113} \mathrm{Sn}<9$.E-1, and ${ }^{152} \mathrm{Eu}<3$.E-1. The detection limits for the AZ-102 Melt 1 samples in $\mu \mathrm{Ci} / \mathrm{g}$ are: ${ }^{51} \mathrm{Cr}<5 . \mathrm{E}+0,{ }^{59} \mathrm{Fe}<3 . \mathrm{E}-1,{ }^{95} \mathrm{Nb}<2 . \mathrm{E}-1,{ }^{103} \mathrm{Ru}<6 . \mathrm{E}-1,{ }^{113} \mathrm{Sn}<8$.E-1, and ${ }^{152} \mathrm{Eu}<5$.E-1. Slight gamma activity was detected in the preparation blank.

Stontium-90. Aliquots of the diluted SAL preparation were taken for ${ }^{90} \mathrm{Sr}$ analysis. Along with the C-104 and AZ-102 Melt 1 glass samples, a chemistry sample replicate (00-2313Rep), a chemistry blank (Blank), a reagent spiked with ${ }^{90} \mathrm{Sr}$ (Blank Spike), and a sample spiked with ${ }^{90} \mathrm{Sr}$ (Matrix Spike) were also prepared (see Appendix C). These QC samples help verify the accuracy of the sample separation, counting, and analysis methods. All measures of agreement indicate good precision. The sample and sample replicate agreed to within 1\% RPD. The C-104 sample and the duplicate (prepared in the SAL) agreed to within 9\% RPD. The AZ-102 Melt 1 sample and duplicate agreed to within 4\%. Both the blank spike and matrix spike resulted in $92 \%$ recovery, well within the blank spike limits established by the analytical QA plan of $80-110 \%$. The process blank resulted in non-detectable ${ }^{90} \mathrm{Sr}$ thus indicating processing contamination was not measurable.

Plutonium-239/240 and Plutonium-241. Plutonium was isolated from diluted SAL preparations. The separated fractions were then precipitation plated and counted by alpha spectrometry. After alpha-counting was complete, the filters were suspended in scintillation cocktail and beta-counted. The

${ }^{241} \mathrm{Pu}$ count rate was determined by integrating from $2-20 \mathrm{keV}$. The liquid scintillation counter was calibrated relative to tritium that has a similar beta end-point energy $(18.6 \mathrm{keV})$. 
QC measures similar to that of ${ }^{90} \mathrm{Sr}$ determination were used for $\mathrm{Pu}$ analysis. A sample replicate was used to monitor separations and counting precision, whereas the sample duplicate measures the precision of the entire process (including SAL preparations). Measures of agreement indicate good precision for ${ }^{239 / 240} \mathrm{Pu}$. The sample replicate RPD was $4 \%$, the C-104 sample and duplicate RPD was $2 \%$. The AZ-102 Melt 1 RPD was a little higher at 11\% but well within the $20 \%$ RPD criterion of the QA plan. Blank spike recovery, $112 \%$, exceeded the acceptance criterion of $80-110 \%$. The matrix spike recovery, $109 \%$, was within the QA plan criterion of $75 \%$ to $125 \%$.

Additional $\mathrm{Pu}$ isotopes, ${ }^{238} \mathrm{Pu}$ and ${ }^{236} \mathrm{Pu}$, were measured opportunistically with the ${ }^{239 / 240} \mathrm{Pu}$ and are reported for additional information.

Measures of agreement for ${ }^{241} \mathrm{Pu}$ approached and exceeded the $20 \%$ RPD bounds defined in the QA plan (23\% RPD between the C-104 sample and replicate, 16\% RPD between the C-104 sample and duplicate, and 22\% between the AZ-102 Melt 1 sample and duplicate). To further evaluate the precision, the mean difference calculation was applied and is reported. If the mean difference exceeds 1.96 , the reported values are considered truly different. If the mean difference is less than 1.96 , then, within the error of the measurements, the values are in agreement. In this case the mean differences were 1.15 and 1.23 , indicating the reported results are in agreement. The blank spike and matrix spike recoveries were $80 \%$ and $75 \%$, respectively, meeting the QA plan acceptance criteria. The recoveries were lower than typically found in ${ }^{239} \mathrm{Pu}$ spike recoveries. This may be due in part to the geometry of the filter and lowenergy beta attenuation in the filter.

The process blank ${ }^{239 / 240} \mathrm{Pu}$ and ${ }^{241} \mathrm{Pu}$ concentrations were about three orders of magnitude less than the sample concentrations, indicating little hot cell and processing contamination occurred relative to these isotopes.

Uranium. Total uranium was determined on dilutions of the SAL preparations using kinetic phosphorescence analysis. All RPDs were 5\%, well within the $20 \%$ acceptance criterion. The process blank was nominally 4 orders of magnitude lower in U concentration than the samples, indicating no relevant $U$ contamination occurred during processing. The sample spike recovery of $114 \%$ was well within the matrix spike acceptance criterion of $75-125 \%$.

\section{$\underline{\text { ICP-MS Radionuclide Analyses }}$}

The following radioisotopes: ${ }^{99} \mathrm{Tc},{ }^{237} \mathrm{~Np},{ }^{239} \mathrm{Pu},{ }^{240} \mathrm{Pu},{ }^{233} \mathrm{U},{ }^{234} \mathrm{U},{ }^{235} \mathrm{U},{ }^{236} \mathrm{U}$ and ${ }^{238} \mathrm{U}$ were measured by inductively coupled plasma mass spectroscopy (ICP-MS) in the C-104, AZ-102 Melt 1, and AZ-102 Melt 2 glasses.

Technetium Isotopic Analysis. The ${ }^{99} \mathrm{Tc}$ values reported assume that the ruthenium present is exclusively fission product ruthenium and therefore does not have an isotope at $\mathrm{m} / \mathrm{z} 99$. The isotopic analysis observed for $\mathrm{Ru}$ is not a natural distribution and is consistent with previous tank waste analysis. A cobalt standard was analyzed to evaluate the ${ }^{59} \mathrm{Co}^{40} \mathrm{Ar}$ interference. Worst case, the CoAr concentration could result in a $1-3 \%$ interference. Therefore, the entire response at $\mathrm{m} / \mathrm{z} 99$ is attributed to ${ }^{99} \mathrm{Tc}$. 
Uranium Isotopic Analysis. The samples were diluted for total uranium analysis; no interference corrections were made since the $\mathrm{Pb}$ concentrations were found to be low. Uranium isotopic analysis requires that the sample matrix (high solids) and polyatomic interferences like $\mathrm{PbN}_{\mathrm{x}} \mathrm{O}_{\mathrm{y}}{ }^{+}$be removed prior to analysis. This separation was accomplished by column extraction using TRU-Spec resin. The separated samples were analyzed for uranium isotopes and the isotopic ratios determined. The isotope ratios were applied to the total uranium concentration to calculate the concentration of each isotope.

Plutonium Isotopic Analysis. The analysis of ${ }^{239} \mathrm{Pu}$ and ${ }^{240} \mathrm{Pu}$ require the removal of uranium and lead. The molecular ion production of $\left({ }^{238} \mathrm{U}^{1} \mathrm{H}\right)^{+}$and $\left({ }^{204} \mathrm{~Pb}^{35} \mathrm{Cl}\right)^{+}$are major interferences to this analysis. This separation is accomplished by column extraction using TEVA-Spec resin. ${ }^{242} \mathrm{Pu}$ was used as a tracer for the plutonium separations. The ICP-MS plutonium values reported are corrected for the tracer recovery, which varied $20-80 \%$. Values for ${ }^{240} \mathrm{Pu}$ were obtained using the response from ${ }^{239} \mathrm{Pu}$. Because

$\mathrm{a}^{240} \mathrm{Pu}$ standard was not available and the concentrations determined indirectly, the ICP-MS ${ }^{240} \mathrm{Pu}$ results should be considered semiquantitative.

\section{HLW Glass Radionuclide Inventory Results}

Tables 4.8 and 4.9 provide radiochemical data from the Envelope D glasses C-104 and AZ-102 in comparison with compositional predictions from the waste. The first column, labeled "Expected Loading," of data provides radionuclide estimates based on sample analysis of the pretreated wastes. The second and third columns of data provide analyzed radionuclide values from the actual waste glasses produced by vitrification of the waste. Percent recoveries in the glass are calculated and presented in the last column. Radiochemical percent recovery is defined herein as the amount of a radionuclide detected in the final glass divided by the expected loading amount for the glass (expected loading values were calculated by summing the HLW pretreated sludge waste, Cs and Tc ion exchange eluants, and Sr/TRU precipitate waste values and converting to a glass gram basis). The waste percent recovery values for the C-104 and AZ-102 Melt 1 glasses are generally in line with expectations. Am/Cm recoveries were in the 75 to $100 \%$ range. Yields for $\mathrm{Pu}$ were within the expected range of approximately $80 \%$. Some ${ }^{238} \mathrm{Pu}$, but no $\mathrm{Cm}$ was detected. However, the C-104 glass recoveries for ${ }^{154} \mathrm{Eu}$ and ${ }^{155} \mathrm{Eu}$ appear to be high by a factor of two. 
Table 4.8. Radiochemical Composition of C-104 Glass

\begin{tabular}{|c|c|c|c|c|}
\hline \multirow{2}{*}{ C-104 Glass } & \multirow{2}{*}{$\begin{array}{c}\text { Expected }^{(a)} \\
\text { Loading } \\
(\mu \mathrm{Ci} / \text { g glass })\end{array}$} & \multicolumn{2}{|c|}{$\begin{array}{c}\text { Analysis of Glass } \\
(\mu \mathrm{Ci} / \mathrm{g} \text { glass })\end{array}$} & \multirow{2}{*}{$\begin{array}{c}\text { Waste Recovery } \\
\text { in Glass (\%) }\end{array}$} \\
\hline & & Radiochemistry & ICP-MS & \\
\hline Cr-51 & & $<5.0$ & & \\
\hline Fe-59 & & $<0.1$ & & \\
\hline Co-60 & 0.307 & 0.127 & & 41.3 \\
\hline Sr-90 & 518 & 519 & & 100.2 \\
\hline $\mathrm{Nb}-95$ & & $<0.06$ & & \\
\hline Tc-99 & 0.014 & $\mathrm{NM}$ & 0.0125 & 89.3 \\
\hline $\mathrm{Ru}-103$ & & $<0.06$ & & \\
\hline $\mathrm{Ru}-106$ & & $<4.0$ & & \\
\hline Sn-113 & & $<0.9$ & & \\
\hline Sb-125 & 0.13 & $<3.0$ & & \\
\hline Cs-134 & 0.16 & 0.12 & & 75 \\
\hline Cs-137 & 1280 & 1160 & & 90.6 \\
\hline Ce-144 & & $<3.0$ & & \\
\hline Eu-152 & & $<0.3$ & & \\
\hline Eu-154 & 1.25 & 2.68 & & 214.4 \\
\hline Eu-155 & 0.73 & 1.30 & & 178.1 \\
\hline $\mathrm{Pu}-236$ & & $<0.005$ & & \\
\hline $\mathrm{Np}-237$ & 0.004 & NM & 0.00298 & 74.5 \\
\hline $\mathrm{Pu}-238$ & & 0.443 & & \\
\hline $\mathrm{Pu}-239+\mathrm{Pu}-240$ & 4.05 & 3.33 & 1.652 & 82.5 \\
\hline $\mathrm{Pu}-241$ & & 11.6 & & \\
\hline $\mathrm{Am}-241$ & 4.68 & 4.8 & & 103 \\
\hline $\mathrm{Cm}-242$ & 0.0065 & NM & & \\
\hline $\mathrm{Cm}-243+\mathrm{Cm}-244$ & 0.069 & $\mathrm{NM}$ & & \\
\hline \multicolumn{5}{|c|}{$\begin{array}{l}\text { (a) Radionuclide composition of glass is estimated from the waste compositional analysis and does } \\
\text { not account for volatilization. Actual quantities of } \mathrm{Cs} \text { and Tc in the glass are expected to be } \\
\text { lower, due to volatilization. } \\
<\mathrm{x} . \mathrm{xx}=\text { indicates that the radioisotope is below the detection limit, detection limit value is provided } \\
\text { for those radioisotopes. } \\
\mathrm{NM}=\text { not measured }\end{array}$} \\
\hline
\end{tabular}


Table 4.9. Radiochemical Composition of AZ-102 Glasses

\begin{tabular}{|c|c|c|c|c|c|}
\hline \multirow{3}{*}{$\begin{array}{c}\text { AZ-102 Melt } 1 \\
\text { (except as noted) }\end{array}$} & \multirow{3}{*}{ 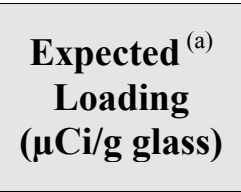 } & \multicolumn{3}{|c|}{$\begin{array}{l}\text { Analysis of Glass } \\
(\mu \mathrm{Ci} / \mathrm{g} \text { glass })\end{array}$} & \multirow{3}{*}{$\begin{array}{c}\text { Waste Recovery } \\
\text { in Glass (\%) }\end{array}$} \\
\hline & & \multirow[b]{2}{*}{ Radiochemistry } & \multicolumn{2}{|c|}{ ICP-MS } & \\
\hline & & & Melt 1 & Melt 2 & \\
\hline Cr-51 & & $<<5.0$ & & & \\
\hline Fe-59 & & $<0.3$ & & & \\
\hline Co-60 & 3.07 & 2.67 & & & 87 \\
\hline Se-79 & & NM & & & \\
\hline$Y-88$ & & NM & & & \\
\hline Sr-90 & 11800 & 8900 & & & 75 \\
\hline $\mathrm{Nb}-95$ & & $<0.2$ & & & \\
\hline Tc-99 & & NM & 0.00939 & 0.0154 & \\
\hline Ru-103 & & $<0.6$ & & & \\
\hline Ru-106 & & $<0.4$ & & & \\
\hline Sn-113 & & $<0.8$ & & & \\
\hline $\mathrm{Sb}-125$ & 16.1 & 20.9 & & & 130 \\
\hline SnSb-126 & & NM & & & \\
\hline Cs-134 & 0.098 & $<0.3$ & & & \\
\hline Cs-137 & 847 & 705 & & & 83.2 \\
\hline $\mathrm{Ce}-144$ & & $<4.0$ & & & \\
\hline Eu-152 & & $<0.5$ & & & \\
\hline Eu-154 & 29.1 & 26.2 & & & 90 \\
\hline Eu-155 & 53.6 & 46.6 & & & 87 \\
\hline Pu-236 & & $<0.03$ & & & \\
\hline $\mathrm{Np}-237$ & & NM & 0.0356 & 0.0344 & \\
\hline Pu-238 & 0.64 & 0.44 & & & 69 \\
\hline $\mathrm{Pu}-239+\mathrm{Pu}-240$ & 3.96 & 3.37 & 1.468 & 1.669 & 85 \\
\hline $\mathrm{Pu}-241$ & & 16.1 & & & \\
\hline Am-241 & 81.6 & 71.5 & & & 88 \\
\hline $\mathrm{Cm}-242$ & & NM & & & \\
\hline $\mathrm{Cm}-243+\mathrm{Cm}-244$ & 0.12 & NM & & & \\
\hline \multicolumn{6}{|c|}{$\begin{array}{l}\text { (a) Radionuclide composition of glass is estimated from the waste compositional analysis and does not } \\
\text { account for volatilization. Actual quantities of } \mathrm{Cs} \text { and Tc in the glass are expected to be lower, due to } \\
\text { volatilization. } \\
\text { <x.xx = indicates that the radioisotope is below the detection limit, detection limit value is provided for those } \\
\text { radioisotopes. } \\
\mathrm{NM}=\text { not measured }\end{array}$} \\
\hline
\end{tabular}

Some of the primary success objectives for this work were that for the IHLW glasses the following had to be reported: 1) "the inventory of radionuclides (in Curies) that have half-lives longer than 10 years and that are, or will be, present in concentrations greater than 0.05 percent of the total radioactive inventory for each waste type, indexed to the years 2015 and 3115."; 2) "The total and fissile uranium and plutonium content of each canister in grams."; 3 ) "The concentration of plutonium in grams per cubic meter for each canister." and in addition "The concentration of plutonium in each HLW standard canister shall be less than 2,500 grams/cubic meter."; and 4) "The ratio by weight of the total element of the following isotopes: U-233, U-234, U-235, U-236, U-238, Pu-238, Pu-239, Pu-240, Pu-241, and Pu-242." It has been assumed that each HLW glass, i.e. C-104, AZ-102 Melt 1, and AZ-102 Melt 2, are separate "waste types" and as such would fill multiple Hanford HLW canisters and that the HLW canister can be modeled as a right circular cylinder of $4.5 \mathrm{~m}$ height and $0.61 \mathrm{~m}$ diameter with a $100 \%$ glass fill of approximately $1.27 \mathrm{~m}^{3}$. 
Tables 4.10. 4.11, 4.12, and 4.13 combined provide the inventory of radionuclides (in Curies) that have half-lives longer than 10 years and that are, or will be, present in concentrations greater than 0.05 percent of the total radioactive inventory for each waste type, i.e. for each individual glass, indexed to the years 2015 and 3115. equation:

The radioactive decay to the years 2015 and 3115 was calculated using the standard decay

$$
N=N_{0} e^{-\lambda t}
$$

where $N_{0}$ is the number of radioactive atoms at the present time,

$N$ is the number after a specific elapsed time $t$, and

$\lambda$ is 0.683 divided by the half-life of the radioactive isotope.

The standard decay equation can then be written in the form:

$$
N / N_{0}=e^{-0.683 t / t_{1 / 2}}
$$

which is the form used to make the calculations for Table 4.10. The factors in Table 4.10 were used to make the calculations for Tables 4.11, 4.12, and 4.13.

Table 4.10. Radioisotopes with Half-Lives Longer than 10 Years and Half-Life Factors Indexed to years 2015 and 3115

\begin{tabular}{|l|c|c|c|c|}
\hline \multicolumn{1}{|c|}{ Isotope } & Half - Life $\mathbf{1 0 y}$ & $\mathbf{2 0 0 0}$ & $\mathbf{2 0 1 5}$ & $\mathbf{3 1 1 5}$ \\
\hline \hline $\mathrm{Sr}-90$ & $28.5 \mathrm{y}$ & 1 & 0.698 & 0 \\
\hline $\mathrm{Tc}-99$ & $213000 \mathrm{y}$ & 1 & 0.99995 & 0.9964 \\
\hline $\mathrm{Cs}-137$ & $30 \mathrm{y}$ & 1 & 0.711 & 0 \\
\hline $\mathrm{Eu}-152$ & $13.33 \mathrm{y}$ & 1 & 0.4637 & 0 \\
\hline $\mathrm{U}-233$ & $159220 \mathrm{y}$ & 1 & 0.999936 & 0.99523 \\
\hline $\mathrm{U}-234$ & $245460 \mathrm{y}$ & 1 & 0.999958 & 0.99690 \\
\hline $\mathrm{U}-235$ & $7.037 \mathrm{E}+08 \mathrm{y}$ & 1 & 0.999999985 & 0.9999989 \\
\hline $\mathrm{U}-236$ & $2.3423 \mathrm{E}+07 \mathrm{y}$ & 1 & 0.99999956 & 0.999967 \\
\hline $\mathrm{U}-238$ & $4.4685 \mathrm{E}+09 \mathrm{y}$ & 1 & 0.9999999977 & 0.99999983 \\
\hline $\mathrm{Np}-237$ & $2140000 \mathrm{y}$ & 1 & 0.999995 & 0.9996 \\
\hline $\mathrm{Pu}-238$ & $87.7 \mathrm{y}$ & 1 & 0.8897 & 0 \\
\hline $\mathrm{Pu}-239$ & $24110 \mathrm{y}$ & 1 & 0.9996 & 0.9689 \\
\hline $\mathrm{Pu}-240$ & $6560 \mathrm{y}$ & 1 & 0.9984 & 0.8904 \\
\hline $\mathrm{Pu}-241$ & $14.4 \mathrm{y}$ & 1 & 0.4909 & 0 \\
\hline $\mathrm{Pu}-242$ & $376000 \mathrm{y}$ & 1 & 0.99997 & 0.9980 \\
\hline $\mathrm{Am}-241$ & $432.7 \mathrm{y}$ & 1 & 0.9766 & 0.1720 \\
\hline $\mathrm{Am}-242$ & $141 \mathrm{y}$ & 1 & 0.9299 & 0.0045 \\
\hline $\mathrm{Cm}-243$ & $28.5 \mathrm{y}$ & 1 & 0.698 & 0 \\
\hline $\mathrm{Cm}-244$ & $18.11 \mathrm{y}$ & 1 & 0.5680 & 0 \\
\hline
\end{tabular}


Table 4.11. Radioisotopes with Half-Lives Longer than 10 Years Indexed to years 2015 and 3115 for HLW Glass C-104

\begin{tabular}{|l|c|c|c|}
\hline \multicolumn{5}{|c|}{ Glass $\mathbf{C - 1 0 4}$} \\
\hline \multicolumn{1}{|c|}{ Isotope } & $\mathbf{2 0 0 0}(\boldsymbol{\mu C} \mathbf{i} / \mathbf{g})$ & $\mathbf{2 0 1 5}(\boldsymbol{\mu C i} / \mathbf{g})$ & $\mathbf{3 1 1 5}(\boldsymbol{\mu C i} / \mathbf{g})$ \\
\hline \hline $\mathrm{Sr}-90$ & 519 & 362 & 0 \\
\hline $\mathrm{Tc}-99$ & 0.0125 & 0.0125 & 0.0125 \\
\hline $\mathrm{Cs}-137$ & 1160 & 824.8 & 0 \\
\hline $\mathrm{Eu}-152$ & $<0.3$ & $<0.139$ & 0 \\
\hline $\mathrm{Np}-237$ & 0.00298 & 0.00298 & 0.00298 \\
\hline $\mathrm{U}-233$ & 0.328 & 0.328 & 0.328 \\
\hline $\mathrm{U}-234$ & 0.0137 & 0.0137 & 0.0137 \\
\hline $\mathrm{U}-235$ & 0.000468 & 0.000468 & 0.000468 \\
\hline $\mathrm{U}-236$ & 0.000627 & 0.000627 & 0.000627 \\
\hline $\mathrm{U}-238$ & 0.00978 & 0.00978 & 0.00978 \\
\hline $\mathrm{Pu}-238$ & 0.443 & 0.396 & 0 \\
\hline $\mathrm{Pu}-239$ & 2.425 & 2.424 & 2.350 \\
\hline $\mathrm{Pu}-240$ & 0.905 & 0.904 & 0.806 \\
\hline $\mathrm{Pu}-241$ & 11.6 & 5.69 & 0 \\
\hline $\mathrm{Pu}-242$ & $\mathrm{NM}$ & - & - \\
\hline $\mathrm{Am}-241$ & 4.8 & 4.69 & 0.826 \\
\hline $\mathrm{Am}-242$ & $\mathrm{NM}$ & - & - \\
\hline $\mathrm{Cm}-243$ & $\mathrm{NM}$ & - & - \\
\hline $\mathrm{Cm}-244$ & $\mathrm{NM}$ & - & \\
\hline $\mathrm{NM}=$ not measured & \multicolumn{5}{|l}{} \\
\hline
\end{tabular}

Table 4.12. Radioisotopes with Half-Lives Longer than 10 Years Indexed to years 2015 and 3115 for HLW Glass AZ-102 Melt 1

\begin{tabular}{|l|c|c|c|}
\hline \multicolumn{5}{|c|}{ AZ-102 Melt 1 } \\
\hline \multicolumn{1}{|c|}{ Isotope } & $\mathbf{2 0 0 0}(\boldsymbol{\mu C} \mathbf{i} / \mathbf{g})$ & $\mathbf{2 0 1 5}(\boldsymbol{\mu C i} / \mathbf{g})$ & $\mathbf{3 1 1 5}(\boldsymbol{\mu C i} / \mathbf{g})$ \\
\hline Sr-90 & 8900 & 6212 & 0 \\
\hline $\mathrm{Tc}-99$ & 0.00939 & 0.00939 & 0.00936 \\
\hline $\mathrm{Cs}-137$ & 705 & 501 & 0 \\
\hline $\mathrm{Eu}-152$ & $<0.5$ & $<0.232$ & 0 \\
\hline $\mathrm{Np}-237$ & 0.0356 & 0.0356 & 0.0356 \\
\hline $\mathrm{U}-233$ & 0.0062 & 0.0062 & 0.0062 \\
\hline $\mathrm{U}-234$ & 0.00688 & 0.00688 & 0.00686 \\
\hline $\mathrm{U}-235$ & 0.000278 & 0.000278 & 0.000278 \\
\hline $\mathrm{U}-236$ & 0.000498 & 0.000498 & 0.000498 \\
\hline $\mathrm{U}-238$ & 0.00506 & 0.00506 & 0.00506 \\
\hline $\mathrm{Pu}-238$ & 0.44 & 0.391 & 0 \\
\hline $\mathrm{Pu}-239$ & 2.583 & 2.582 & 2.503 \\
\hline $\mathrm{Pu}-240$ & 0.787 & 0.786 & 0.701 \\
\hline $\mathrm{Pu}-241$ & 16.1 & 7.90 & 0 \\
\hline $\mathrm{Pu}-242$ & $\mathrm{NM}$ & - & - \\
\hline $\mathrm{Am}-241$ & 71.5 & 69.8 & 12.3 \\
\hline $\mathrm{Am}-242$ & $\mathrm{NM}$ & - & - \\
\hline $\mathrm{Cm}-243$ & $\mathrm{NM}$ & - & - \\
\hline $\mathrm{Cm}-244$ & $\mathrm{NM}$ & - & \\
\hline $\mathrm{NM}=$ not measured &
\end{tabular}


Table 4.13. Radioisotopes with Half-Lives Longer than 10 Years Indexed to years 2015 and 3115 for HLW Glass AZ-102 Melt 2

\begin{tabular}{|c|c|c|c|}
\hline \multicolumn{4}{|c|}{ AZ-102 Melt 2} \\
\hline Isotope & $2000(\mu \mathrm{Ci} / \mathrm{g})$ & $2015(\mu \mathrm{Ci} / \mathrm{g})$ & $3115(\mu \mathrm{Ci} / \mathrm{g})$ \\
\hline Sr-90 & NM & - & - \\
\hline Tc-99 & 0.0154 & 0.0154 & 0.0153 \\
\hline Cs-137 & NM & - & - \\
\hline Eu-152 & NM & - & - \\
\hline Np-237 & 0.0344 & 0.0344 & 0.0344 \\
\hline U-233 & 0.0096 & 0.0096 & 0.0096 \\
\hline U-234 & 0.00652 & 0.00652 & 0.00650 \\
\hline U-235 & 0.000265 & 0.000265 & 0.000265 \\
\hline U-236 & 0.000469 & 0.000469 & 0.000469 \\
\hline U-238 & 0.00507 & 0.00507 & 0.00507 \\
\hline Pu-238 & NM & - & - \\
\hline Pu-239 & 2.574 & 2.573 & 2.494 \\
\hline Pu-240 & 0.796 & 0.795 & 0.709 \\
\hline Pu-241 & NM & - & - \\
\hline $\mathrm{Pu}-242$ & NM & - & - \\
\hline Am-241 & NM & - & - \\
\hline Am-242 & NM & - & - \\
\hline $\mathrm{Cm}-243$ & NM & - & - \\
\hline $\mathrm{Cm}-244$ & NM & - & - \\
\hline $\mathrm{NM}=$ not & & & \\
\hline
\end{tabular}

Table 4.14 presents ICP-MS data obtained for the uranium isotopes and ${ }^{239} \mathrm{Pu}$ and ${ }^{240} \mathrm{Pu}$ contained in the C-104, AZ-102 Melt 1, and AZ-102 Melt 2 glasses. Table 4.14 data along with the data provided in Table 4.15 allow the total and fissile uranium and plutonium (U-233, U-235, Pu-239, and Pu-241) content of each canister of waste glass in grams to be calculated. The total and fissile uranium and plutonium (U-233, U-235, Pu-239, and Pu-241) content of each canister of waste glass are: 1) 1161.5 grams for C-104; 2) 642.1 grams for AZ-102 Melt 1; and 3) 619.4 grams for AZ-102 Melt 2.

Table 4.14 data along with the data provided in Table 4.16 allow the concentration of plutonium in grams per cubic meter of waste glass to be calculated. The concentration of plutonium in grams per cubic meter of each waste glass are: 1) $124.7 \mathrm{~g} / \mathrm{m}^{3}$ for C-104; 2) $122.4 \mathrm{~g} / \mathrm{m}^{3}$ for AZ-102 Melt 1; and 3) $122.1 \mathrm{~g} / \mathrm{m}^{3}$ for AZ-102 Melt 2, none of which exceed the contract plutonium loading of 2500 grams per cubic meter. 
Table 4.14. ICP-MS Data Providing the ${ }^{99} \mathrm{Tc}$, Uranium Isotopic, ${ }^{237} \mathrm{~Np},{ }^{239} \mathrm{Pu}$, and ${ }^{240} \mathrm{Pu}$ Glass Content for Each of the HLW Glasses

\begin{tabular}{|l|c|c|c|c|c|c|c|}
\hline \multicolumn{2}{|c|}{ Glass } & \multicolumn{2}{c|}{$\mathbf{C - 1 0 4}$} & \multicolumn{2}{c|}{ AZ-102 Melt 1 } & \multicolumn{2}{c|}{ AZ-102 Melt 2 } \\
\hline Isotope & Spec. Act. ${ }^{\mathbf{a}}-\mathbf{C i} / \mathbf{g}$ & $\boldsymbol{\mu C \mathbf { C } / \mathbf { g }}$ & $\boldsymbol{\mu g} / \mathbf{g}$ & $\boldsymbol{\mu C i} / \mathbf{g}$ & $\boldsymbol{\mu g} / \mathbf{g}$ & $\boldsymbol{\mu C i} / \mathbf{g}$ & $\boldsymbol{\mu g} / \mathbf{g}$ \\
\hline \hline${ }^{99} \mathrm{Tc}$ & 0.0170 & 0.0125 & 0.735 & 0.00939 & 0.552 & 0.0154 & 0.906 \\
\hline${ }^{233} \mathrm{U}$ & 0.00964 & 0.328 & 34.025 & 0.0062 & 0.643 & 0.0096 & 0.996 \\
\hline${ }^{234} \mathrm{U}$ & 0.006225 & 0.0137 & 2.201 & 0.00688 & 1.105 & 0.00652 & 1.047 \\
\hline${ }^{235} \mathrm{U}$ & 0.000001922 & 0.000468 & 243.5 & 0.000278 & 144.64 & 0.000265 & 137.88 \\
\hline${ }^{236} \mathrm{U}$ & 0.00006508 & 0.000627 & 9.634 & 0.000498 & 7.652 & 0.000469 & 7.207 \\
\hline${ }^{238} \mathrm{U}$ & 0.0000003358 & 0.00978 & 29100. & 0.00506 & 15100 & 0.00507 & 15100. \\
\hline Total U & - & - & 29400 & - & 15300 & - & 15200 \\
\hline${ }^{237} \mathrm{~Np}$ & 0.000705 & 0.00298 & 4.227 & 0.0356 & 50.50 & 0.0344 & 48.79 \\
\hline${ }^{239} \mathrm{Pu}$ & 0.06204 & 1.203 & 19.39 & 1.125 & 18.13 & 1.275 & 20.55 \\
\hline${ }^{240} \mathrm{Pu}$ & 0.22696 & 0.449 & 1.978 & 0.343 & 1.511 & 0.394 & 1.736 \\
\hline${ }^{(a)=}$ The specific activity is taken from Browne and Firestone, 1986. & & & \\
\hline
\end{tabular}

Table 4.15. The Total and Fissile Uranium and Plutonium (U-233, U-235, Pu-239, and Pu-241) Content of Each Glass Waste Type in Grams for a Cubic Meter of Glass

\begin{tabular}{|c|c|c|c|c|c|c|}
\hline & & & \multicolumn{2}{|c|}{ AZ-102 $(\mu \mathrm{g} / \mathrm{g})$} & \multicolumn{2}{|c|}{$A Z-102^{e} \mathrm{~g} / \mathrm{m}^{3}$} \\
\hline Fissile Isotope & C-104 ( $\mu \mathrm{g} / \mathrm{g})$ & $\mathrm{C}-104^{\mathrm{d}} \mathrm{g} / \mathrm{m}^{3}$ & Melt 1 & Melt 2 & Melt 1 & Melt 2 \\
\hline${ }^{233} \mathrm{U}$ & 34.025 & 98.26 & 0.643 & 0.996 & 1.738 & 2.449 \\
\hline${ }^{235} \mathrm{U}$ & 243.5 & 703.2 & 144.6 & 137.9 & 390.9 & 372.7 \\
\hline${ }^{239} \mathrm{Pu}^{\mathrm{a}}$ & 39.06 & 112.8 & 41.62 & 41.49 & 112.5 & 112.1 \\
\hline${ }^{241} \mathrm{Pu}^{\mathrm{b}}$ & 0.113 & 0.3263 & 0.156 & 0.156 & 0.423 & 0.422 \\
\hline Totals $^{c}$ & & 914.6 & & & 505.6 & 487.7 \\
\hline
\end{tabular}

(a) the radiochemistry value was used here because it is twice as large as the ICP-MS value.

(b) the specific activity of ${ }^{241} \mathrm{Pu}$ is $103.0 \mathrm{Ci}$ per gram.

(c) the loading per canister is easily calculated by multiplying the totals by the Hanford HLW canister volume in $\mathrm{m}^{3}$ which is approximately $1.27 \mathrm{~m}^{3}$ at $100 \%$ fill.

(d) density of HLW98-51R glass (which is the equivalent C-104 simulant glass), provided by VSL, was measured at $20^{\circ} \mathrm{C}$ using ASTM D854-83 and is $2.888 \mathrm{~g} / \mathrm{cm}^{3}$.

(e) density of HLW98-61 (which is the equivalent AZ-102 simulant glass), provided by VSL, was measured at $20^{\circ} \mathrm{C}$ using ASTM D $854-83$ and is $2.703 \mathrm{~g} / \mathrm{cm}^{3}$. 
Table 4.16. The Concentration of Plutonium in Grams per Cubic Meter for Each Hanford HLW Canister

\begin{tabular}{|c|c|c|c|c|c|c|}
\hline \multirow{2}{*}{$\begin{array}{l}\text { Plutonium } \\
\text { Isotope }\end{array}$} & \multirow[b]{2}{*}{ C-104 $(\mu \mathrm{g} / \mathrm{g})$} & \multirow[b]{2}{*}{$\mathrm{C}-104^{\mathrm{d}} \mathrm{g} / \mathrm{m}^{3}$} & \multicolumn{2}{|c|}{ AZ-102 $(\mu \mathrm{g} / \mathrm{g})$} & \multicolumn{2}{|c|}{$A Z-102^{\mathrm{e}} \mathrm{g} / \mathrm{m}^{3}$} \\
\hline & & & Melt 1 & Melt 2 & Melt 1 & Melt 2 \\
\hline $\mathrm{Pu}-238^{\mathrm{a}}$ & 0.0260 & 0.0751 & 0.0257 & $0.0257^{\mathrm{f}}$ & 0.0695 & $0.0695^{\mathrm{f}}$ \\
\hline $\mathrm{Pu}-239$ & 39.06 & 112.8 & 41.62 & 41.49 & 112.5 & 112.1 \\
\hline $\mathrm{Pu}-240^{\mathrm{b}}$ & 3.985 & 11.51 & 3.47 & 3.51 & 9.38 & 9.49 \\
\hline $\mathrm{Pu}-241^{\mathrm{a}}$ & 0.113 & 0.3263 & 0.156 & $0.156^{\mathrm{f}}$ & 0.422 & $0.422^{\mathrm{f}}$ \\
\hline $\mathrm{Pu}-242$ & NM & $\mathrm{NM}$ & NM & NM & - & - \\
\hline Totals $^{c}$ & & 124.7 & & & 122.4 & 122.1 \\
\hline \multicolumn{7}{|c|}{ (a) The specific activity of $\mathrm{Pu}-238$ and $\mathrm{Pu}-241$ is 17.119 and $103.0 \mathrm{Ci}$ per gram respectively. } \\
\hline \multicolumn{7}{|c|}{ (b) The radiochemistry value was used here because it is twice as large as the ICP-MS value. } \\
\hline \multicolumn{7}{|c|}{$\begin{array}{l}\text { (c) The loading per canister is easily calculated by multiplying the totals by the Hanford HLW canister volume } \\
\text { in } \mathrm{m}^{3} \text { which is approximately } 1.27 \mathrm{~m}^{3} \text { at } 100 \% \text { fill. }\end{array}$} \\
\hline \multicolumn{7}{|c|}{$\begin{array}{l}\text { (d) The density of HLW98-51R glass (which is the equivalent C-104 simulant glass), provided by VSL, was } \\
\text { measured at } 20^{\circ} \mathrm{C} \text { using ASTM D } 854-83 \text { and is } 2.888 \mathrm{~g} / \mathrm{cm}^{3} \text {. }\end{array}$} \\
\hline \multicolumn{7}{|l|}{ (e) 7} \\
\hline \multicolumn{7}{|c|}{$\begin{array}{l}\text { (f) The value of Pu- } 238 \text { and } \mathrm{Pu}-241 \text { for the AZ-102 Melt } 2 \text { was not measured but assumed to be the same as } \\
\text { that for the AZ-102 Melt } 1 \text {. }\end{array}$} \\
\hline \multicolumn{7}{|c|}{$\mathrm{NM}=$ not measured } \\
\hline
\end{tabular}

Table 4.11 data along with the data provided in Tables 4.14 and 4.15 allow the ratio by weight of the total element of the following isotopes: U-233, U-234, U-235, U-236, U-238, Pu-238, Pu-239, $\mathrm{Pu}-240$, and $\mathrm{Pu}-241$ to be calculated. $\mathrm{Pu}-242$ is not reported as it was used as a tracer for the plutonium separations. Table 4.17 provides the ratio by weight to total weight of the element for the following isotopes: U-233, U-234, U-235, U-236, and U-238.

Table 4.17. The Ratio by Weight to Total Weight of the Element for the Following Isotopes: U-233, $\mathrm{U}-234, \mathrm{U}-235, \mathrm{U}-236$, and U-238

\begin{tabular}{|c|c|c|c|}
\hline Isotope Ratio & $\mathbf{C - 1 0 4}$ & AZ-102 Melt 1 & AZ-102 Melt 2 \\
\hline \hline${ }^{233} \mathrm{U} / \mathrm{U}$ total & 0.00116 & $4.20 \mathrm{E}-05$ & $6.55 \mathrm{E}-05$ \\
\hline${ }^{234} \mathrm{U} / \mathrm{U}$ total & $7.49 \mathrm{E}-05$ & $7.22 \mathrm{E}-05$ & $6.89 \mathrm{E}-05$ \\
\hline${ }^{235} \mathrm{U} / \mathrm{U}$ total & 0.00828 & 0.00945 & 0.00907 \\
\hline${ }^{236} \mathrm{U} / \mathrm{U}$ total & $3.28 \mathrm{E}-04$ & $5.00 \mathrm{E}-04$ & $4.74 \mathrm{E}-04$ \\
\hline${ }^{238} \mathrm{U} / \mathrm{U}$ total & 0.990 & 0.987 & 0.993 \\
\hline
\end{tabular}


Table 4.18. The Ratio by Weight of the Total Element of the Following Isotopes: Pu-238, Pu-239, $\mathrm{Pu}-240$, and Pu-241. Pu-242 is Not Reported As It Was Used As A Tracer For The Plutonium Separations

\begin{tabular}{|c|c|c|}
\hline Isotope Ratio & C-104 & AZ-102 Melt 1 \\
\hline${ }^{238} \mathrm{Pu} / \mathrm{Pu}$ total & 0.0006 & 0.0006 \\
\hline${ }^{239} \mathrm{Pu} / \mathrm{Pu}$ total & 0.9049 & 0.9193 \\
\hline${ }^{240} \mathrm{Pu} / \mathrm{Pu}$ total & 0.0923 & 0.0767 \\
\hline${ }^{241} \mathrm{Pu} / \mathrm{Pu}$ total & 0.0026 & 0.0034 \\
\hline
\end{tabular}

\subsection{Crystalline and Non-Crystalline Phase Determination}

Crystalline and non-crystalline phases were identified and measured using x-ray diffraction (XRD), optical microscopy, and scanning electron microscope (SEM) on glass samples of C-104 and AZ-102 Melt 1 that had been heat-treated as indicated in Table 4.19 to simulate a HLW canister centerline cooling (CCC) curve.

Table 4.19. Temperature Profile Line Segments for the Del Tech Furnace Controller to Generate the Hanford HLW Canister Centerline Cooling Profile

\begin{tabular}{|c|c|c|}
\hline Hours & Temperature $^{\mathbf{0}}{ }^{\mathbf{C})}$ & dT/dt(deg./hr) \\
\hline $0.00-0.17$ & $1004-1050$ & +277 \\
\hline $0.17-2.17$ & $1050-1003$ & -24 \\
\hline $2.2-7.0$ & $1003-844$ & -33 \\
\hline $7.0-10.3$ & $844-749$ & -28.4 \\
\hline $10.3-15.5$ & $749-617$ & -25.5 \\
\hline $15.5-21.2$ & $617-491$ & -22.3 \\
\hline $21.2-25.8$ & $491-400$ & -19.5 \\
\hline
\end{tabular}


XRD was used to analyze the HLW glass samples for crystallinity. As indicated in Figure 4.12 below, both HLW glasses show broad amorphous peaks with only very small crystalline peaks indicated in the AZ-102 Melt 1 sample.

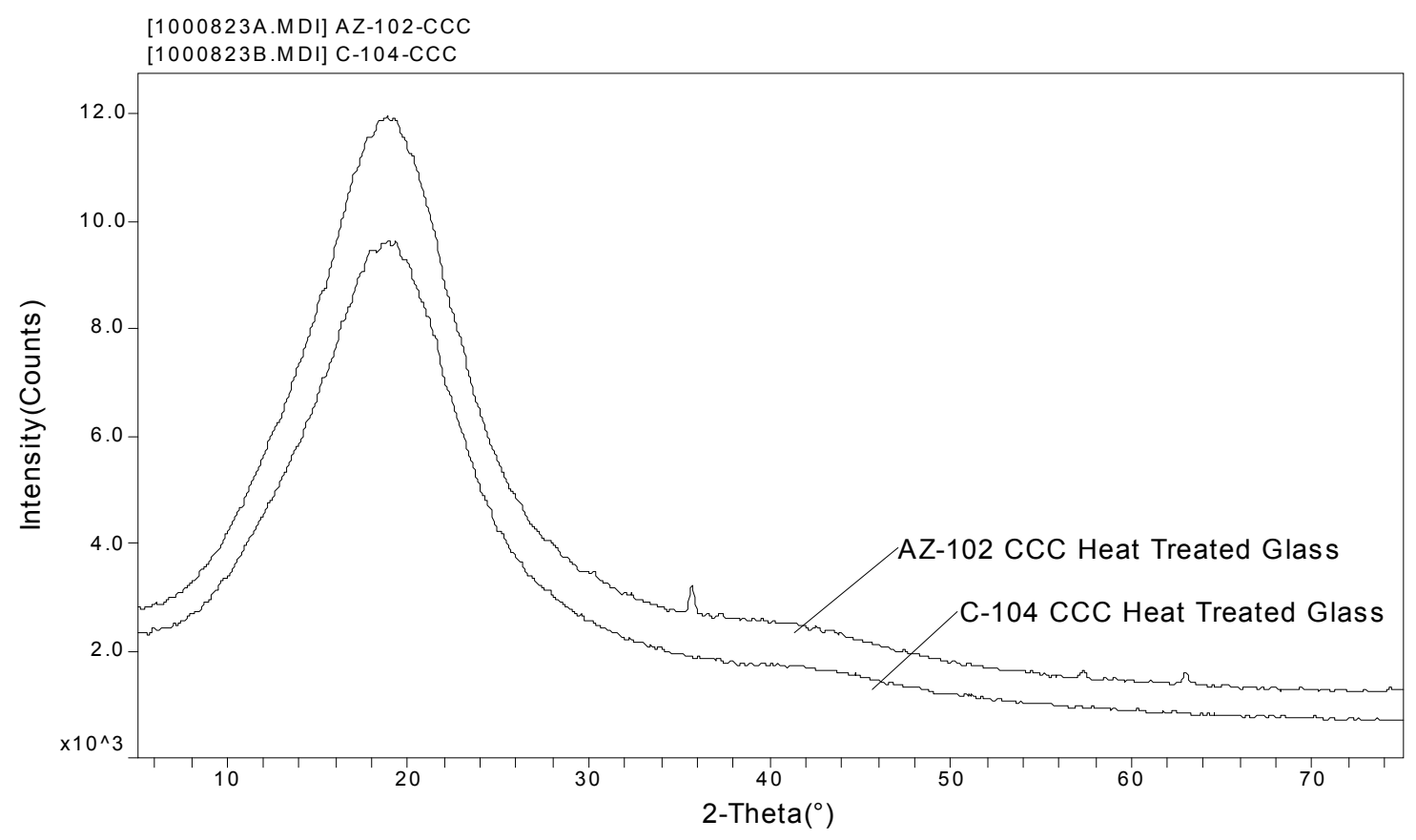

Figure 4.12. These XRD diffraction patterns for HLW glasses C-104 and AZ-102 Melt 1 shows general amorphous characteristics. The small sharp crystalline peaks superimposed on the AZ-102 Melt 1 CCC heat-treated glass diffraction pattern indicate a small amount of a crystalline phase present in this glass

Thin sections of C-104 and AZ-102 Melt 1 CCC heat treated glass samples were prepared and examined in a hot cell with an optical microscope using transmitted and reflected light at magnifications up to $200 \times$ to check for crystalline phases. The C-104 sample was difficult to examine with the optical microscope. Two reasons complicated optical microscopy: one, the sample was opaque, and secondly, the surface of the sample was pitted. The pitting is probably an artifact from grinding and polishing of this particular glass. Those who prepared the C-104 glass sample indicated that it polished differently than the AZ-102 Melt 1 glass and in their words, seemed to be a 'softer' glass, not as 'hard' as the AZ-102 Melt 1 glass. In general, polishing of most HLW glasses to a 600 grit surface finish will give a clear, smooth polished surface. However, in this case, even a 1200 grit polish of the C-104 glass sample still left a very dull, scratched surface (see Appendix D, Figure D.1). Polishing of the C-104 glass sample continued to a 6 micron polish. The final polished surface was much better and reflected light very well, but scratches could still be observed on the surface (see Appendix D, Figure D.2) and a rough appearance on the surface remained especially when observed with the optical microscope (see Appendix D, Figures D.3 and D.4). Analysis by optical microscopy found no crystals or crystalline material contained in the CCC heat-treated C-104 glass sample.

Analysis by optical microscopy of the AZ-102 Melt 1 thin section was difficult using transmitted light due to opacity and crystalline content. Where the sample was thin enough, crystals were observed and digital images taken (see Figure 4.13). Crystals were present in the AZ-102 Melt 1 sample, were 
evenly distributed, with high crystal number density, and the crystal size averaged about 10 microns. Crystals were also observed using reflected light (see Appendix D Figure D.7) and appear as bright spots on the dark surface of the thin section, evenly distributed, numerous, small, and are estimated at 1 to 2 volume percent.

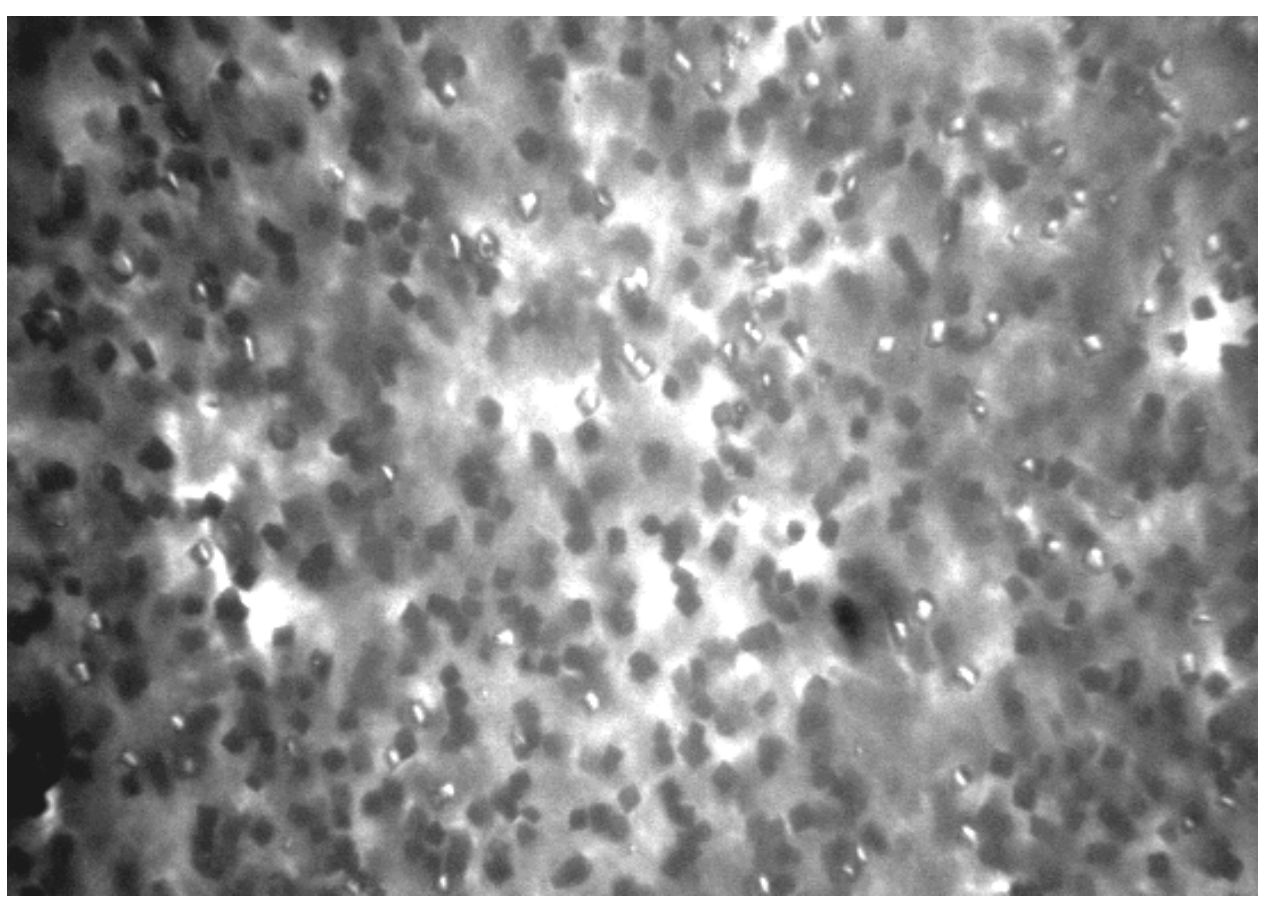

Figure 4.13. An optical microscope was used to examine the AZ-102 Melt 1 CCC heat-treated glass sample at a magnification of $200 \times$ using transmitted light. The picture shows cubic crystals that are evenly dispersed with an average size of about 10 microns

C-104 thin section samples were analyzed by SEM and confirmed the observations made with optical microscopy. For the C-104 thin section, no crystals were observed, but the pitted glass surface was recorded (see Appendix D, Figures D.5 and D.6).

The thin section fragment of the CCC heat-treated AZ-102 Melt 1 glass was also examined using an SEM and found to contain a large number of evenly distributed crystals (see Appendix D and Figure 4.14 below). The elemental composition of the glass surface and some of the crystals were measured in the SEM using energy dispersive spectroscopy (EDS). The crystals had a composition high in oxygen and iron and containing minor amounts of chrome, nickel, and manganese, all major components of a spinel $\left((\mathrm{Fe}, \mathrm{Ni}, \mathrm{Mn})(\mathrm{Fe}, \mathrm{Cr})_{2} \mathrm{O}_{4}\right)$ crystal (see Figure 4.15). Elemental composition of the glass surface was examined in a number of locations and typical peaks from the glass matrix of a HLW glass are shown in Figure 4.16 and as one can see are quite different than the crystal elemental composition.

XRD analysis combined with optical microscopy and SEM EDS results of the CCC heat-treated AZ-102 Melt 1 glass sample indicates the presence of a crystalline phase at approximately 1 to 2 volume percent. An XRD search match analysis of the major peaks found in the XRD pattern of the CCC heattreated AZ-102 Melt 1 glass sample indicated trevorite $\left(\mathrm{NiFe}_{2} \mathrm{O}_{4}\right)$ and chromite $\left(\mathrm{FeCr}_{2} \mathrm{O}_{4}\right)$ as the most likely spinel crystals. The small amount of crystalline material in the CCC heat-treated AZ-102 Melt 1 glass sample does not significantly alter the leaching resistance of the glass as indicated by the PCT test 
results reported in Section 4.3. SEM examination of both the C-104 and AZ-102 Melt 1 CCC heat-treated glasses at magnifications up to $5000 \times$ showed homogenous glasses with no evidence of any phase separation.

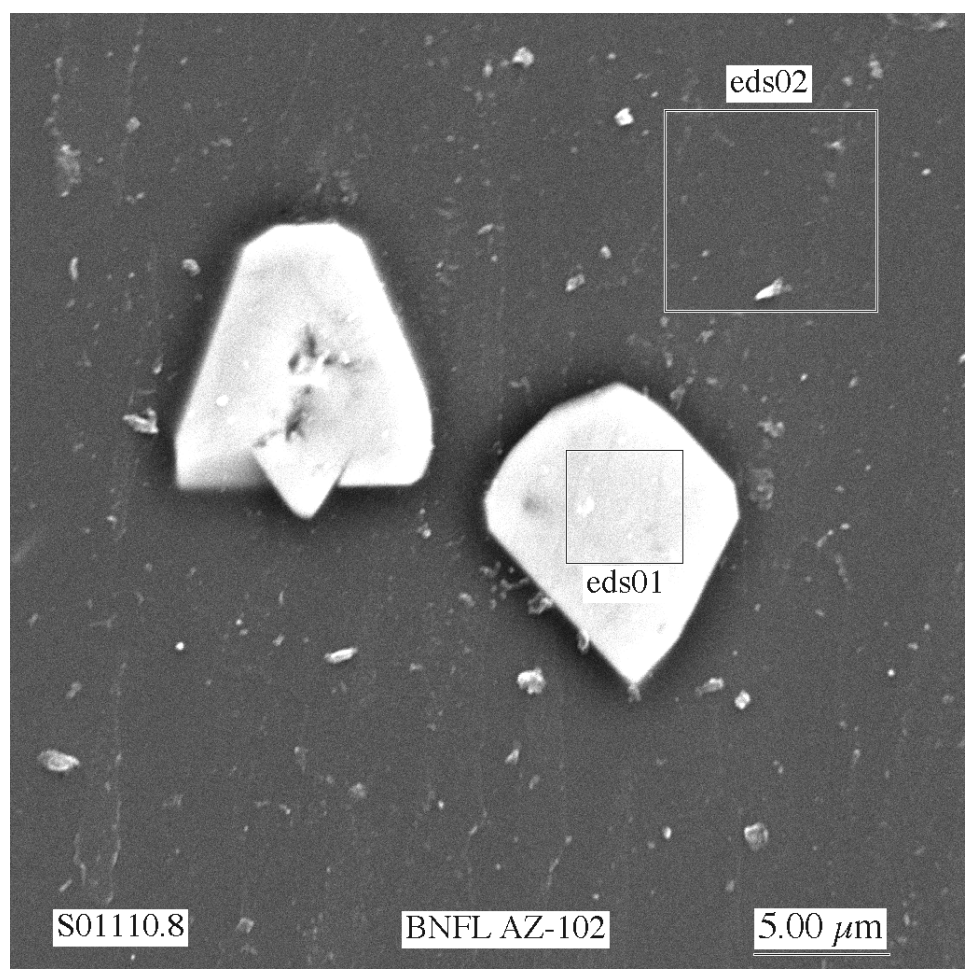

Figure 4.14. SEM photomicrograph, taken at a magnification of $2000 \times$, of the surface of the CCC heat-treated AZ-102 Melt 1 glass thin section. Elemental analysis of a crystal (designated in the picture as "eds01") is provided in Figure 4.15 and of the glass matrix (designated in the picture as "eds02") in

Figure 4.16 


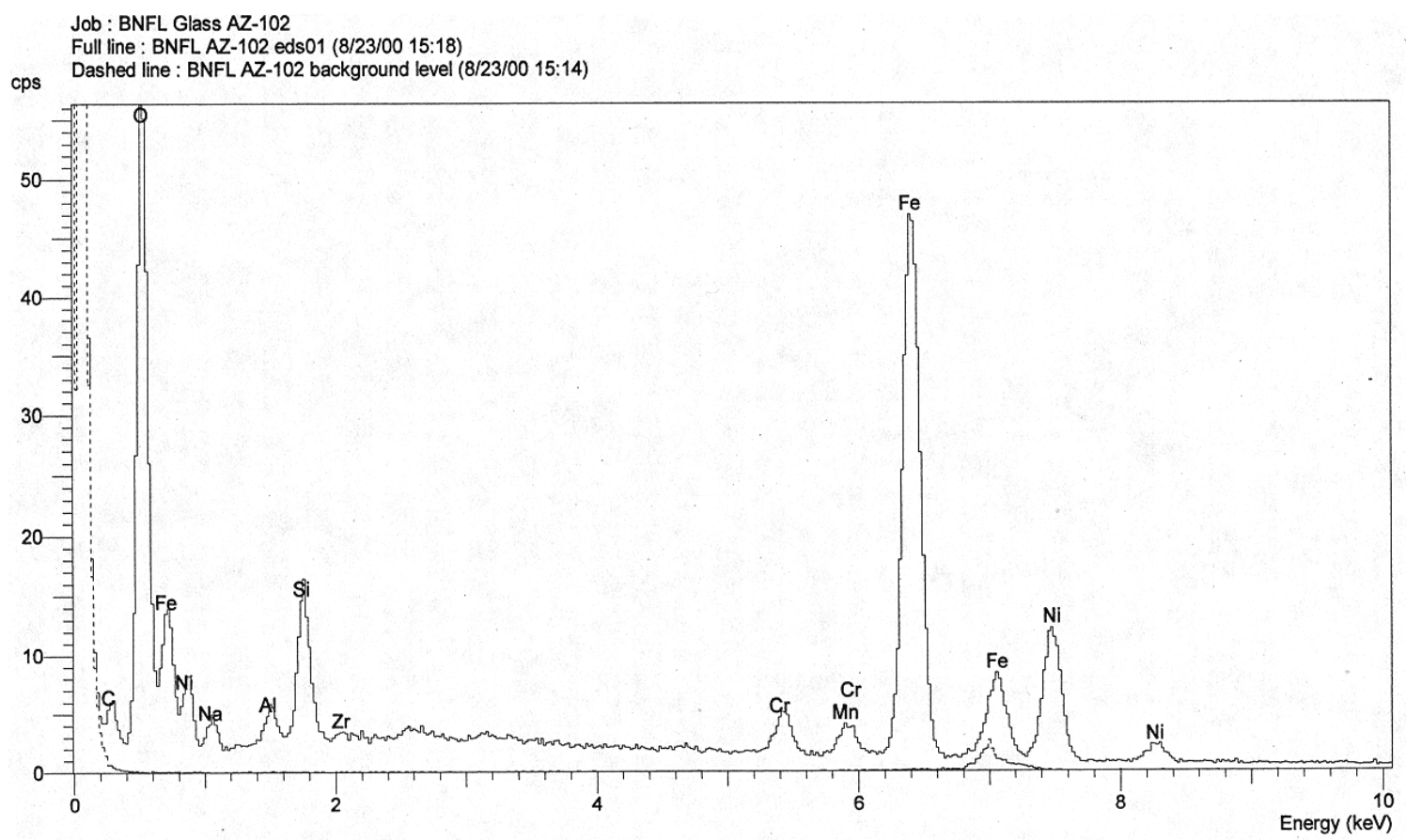

Figure 4.15. EDS scan of the area outlined and labeled "eds01"on the crystal (center right) in the photomicrograph of Figure 4.14. Note the high iron and oxygen peaks combined with moderate nickel and low chrome and manganese peaks, all major components of a spinel $\left((\mathrm{Fe}, \mathrm{Ni}, \mathrm{Mn})(\mathrm{Fe}, \mathrm{Cr})_{2} \mathrm{O}_{4}\right)$ crystal.

Silicon, aluminum, and zirconium peaks are from the glass matrix or impurities within the crystal structure. The carbon is probably from super glue contamination

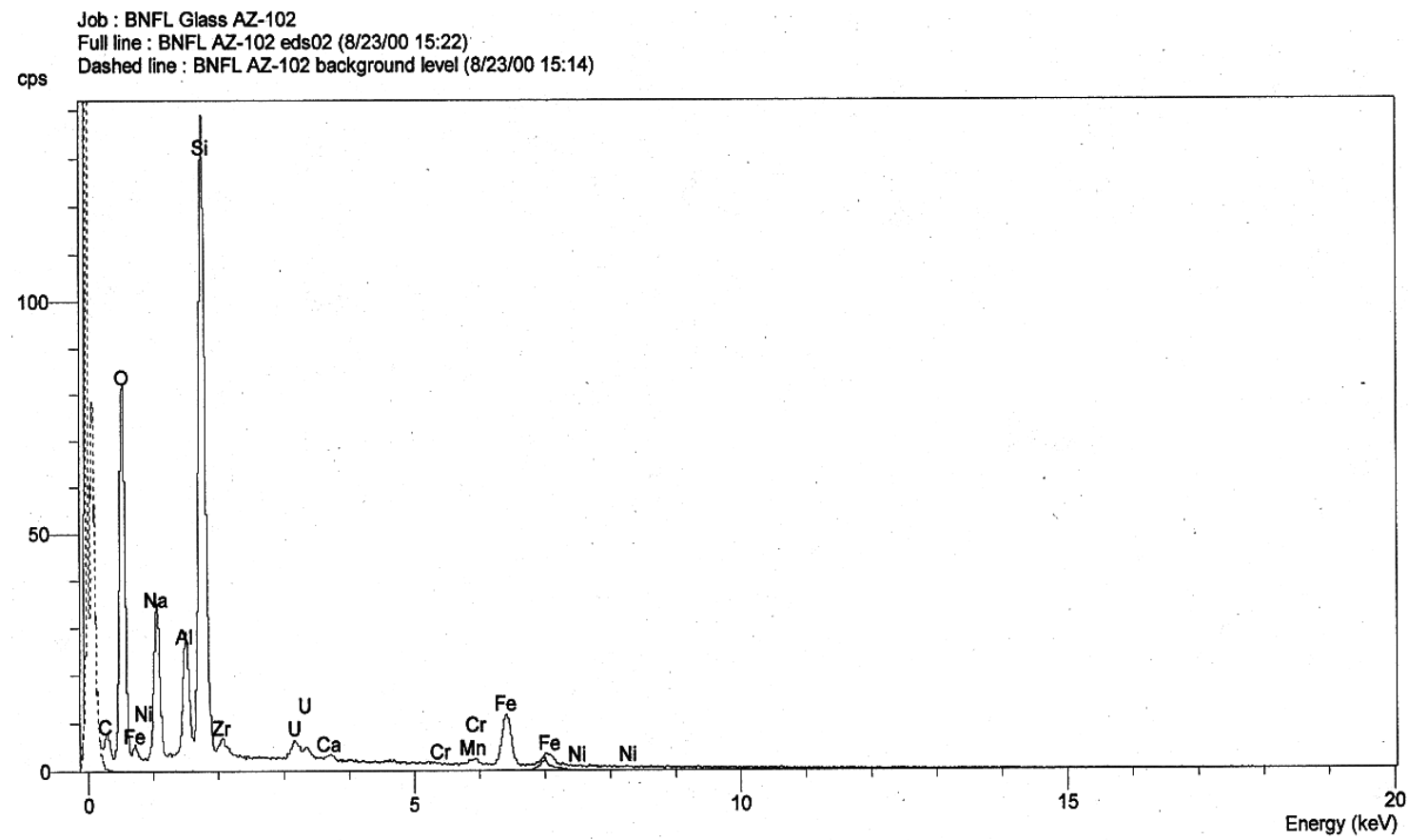

Figure 4.16. EDS scan of the area outlined and labeled "eds02"on the glass surface (upper right corner) in the photomicrograph of Figure 4.14. These are typical peaks from the glass matrix of a HLW glass 


\subsection{Release Rate, Product Consistency Testing of HLW Glasses}

The ultimate objective for immobilization of the high-level radioactive tank waste is to incorporate and convert the radioactive and hazardous components into a solid waste form that will be chemically durable and meet the conditions for storage in a geologic repository for high-level radioactive waste. This resistance of the waste form to release deleterious environmental components is defined by measuring its chemical durability, i.e. the resistance of the glass to react with the aqueous environment expected in the glass disposal site. However, to mimic the mean temperature, amount and frequency of available ground waster, etc. expected in the geologic repository would require a great amount of testing time to be able to detect glass dissolution. Therefore, an accelerated chemical durability test, the Product Consistency Test (PCT) (ASTM C1285-97), is employed to gauge the IHLW glass chemical durability. The glass samples used in the PCT were given a slow cool-down heat treatment (see Section 3.2 for details) which simulates the cooling profile for glass at the center line of a Hanford standard HLW canister being filled with a waste glass and allowed to cool to ambient temperature. The PCT was run at $90^{\circ} \mathrm{C}$ to determine the normalized release of boron, sodium, lithium, aluminum, and silicon. The Environmental Assessment glass (EA glass) test reference material, standard glass (Jantzen et al. 1993) was included in these tests to provide a reliable baseline of results by which to judge the quality of the PCT results for the C-104 and AZ-102 Melt 1 glasses.

After the glass particles were cleaned of adhering fines, the crushed glass (a minimum of $1 \mathrm{~g}$ ) was tested per ASTM C1285-97 "Standard Test Methods for Determining Chemical Durability of Nuclear, Hazardous, and Mixed Waste Glasses: The Product Consistency Test (PCT)." For testing the crushed glass (a minimum of $1 \mathrm{~g}$ ) was placed in a Type $304 \mathrm{~L}$ stainless steel vessel ( $22 \mathrm{~mL}$ volume) into which an amount of ASTM Type I water, equal to $10 \mathrm{~cm}^{3}$ per gram of glass, was added. The vessel was sealed and placed into a constant temperature device at $90 \pm 2{ }^{\circ} \mathrm{C}$. After 7 days, the vessel was cooled to ambient temperature. The $\mathrm{pH}$ was measured on an aliquot of the leachate and the temperature of the aliquot at the time of the $\mathrm{pH}$ measurement was recorded. The remaining leachate was filtered through a $0.45 \mu \mathrm{m}$ filter to remove suspended solids, acidified with Ultrex ${ }^{\circledR}$ nitric acid (high purity) to one volume percent, and sent for analysis. Elemental analyses of boron, alkali metals, aluminum, and silicon concentrations were obtained, which were then used as a measure of the extent of glass corrosion. The concentrations of elements $(\mathrm{C})$ are normalized to glass composition and glass surface area (S) to solution volume (V) according to:

$$
r_{i}=\frac{C_{i}}{f_{i}(S / V)}
$$

where $r_{i}, C_{i}$, and $f_{i}$ are the $i^{\text {th }}$ element normalized release, concentration in solution, and mass fraction in glass, respectively. For PCT (Method A), the glass surface area (S) to solution volume (V) or S/V was calculated to be $2000 \mathrm{~m}^{-1}$ based on assumptions on the size and shape distribution of the ground glass and verified through considerable surface area measurements (ASTM C1285-97).

All tests were run without serious deviation from the PCT procedure. However, the tests were not within the required testing period to be controlled to within $\pm 2 \%$ (168 hours \pm 3.4 hours). To process the samples within the operational time restraints of the HLRF, it was necessary to remove the samples early to have the time needed for sample preparation; therefore, the test period fell short of the minimum requirement of 164.6 hours. Duration of the tests in hours for each PCT sample are: 1) 164.4 (C-104 glass samples); 2) 163.8 (AZ-102 glass samples): 3) 163.7 (EA glass samples); 4) 162.9 (Blank 1); and 5) 179.9 (Blank 2). All samples except the two blanks were within 2.5 percent of the test period. This will have only a small effect on the PCT results, within the error limits of the EA glass reference material analyzed in this test. The deficiency has been reported and evaluated in a Deficiency Report No. DR-BNFL-03. 
Table 4.20 compares the "measured" PCT leachate concentration and $\mathrm{pH}$ with the "round robin" PCT results for the Environmental Assessment (EA) glass as reported by Jantzen et al. 1993. There are some notable differences. The EA glass-1 sample (see Appendix E) was the only test with results encompassed by the reported data of the round robin test. The EA glass- 2 sample fell outside of the lower limits of the reported data while EA glass- 3 sample was almost $50 \%$ below the reported value of boron. The $\mathrm{pH}$ values follow this trend; EA glass- 1 sample is 11.77 (within the reported data values) while the other two samples (11.64 for sample 2 and 11.33 for sample 3) are lower. The chemical analyses have been reviewed with no detectable errors. We believe that the temperature distribution in the oven in the hot cell may be the reason because EA glass- 2 and EA glass-3 samples were closer to the oven walls.

Table 4.21 gives the normalized average release rates found for glasses C-104, AZ-102 Melt 1, and the EA glass at $90^{\circ} \mathrm{C}$. The results of the triplicate samples for the slow cool-down heat-treated glasses C-104 and AZ-102 Melt 1 look very good. These samples are an order of magnitude more durable for the $\mathrm{Si}, \mathrm{Na}$, and $\mathrm{B}$, and just slightly less than an order of magnitude for $\mathrm{Li}$ when compared to the reported results of the EA glass by Jantzen, et al. 1993.

The elemental results of $\mathrm{Al}$ would not have been reported because the elemental release was an insignificant value in the EA and C-104 samples. However, the release of Al in the AZ-102 Melt 1 samples had the highest normalized release of any of the elements analyzed. The average, normalized elemental release for the AZ-102 Melt 1 glass was: $0.226 \pm 0.009 \mathrm{~g} / \mathrm{m}^{2}$ for Al; $0.375 \pm 0.008 \mathrm{~g} / \mathrm{m}^{2}$ for Li; $0.396 \pm 0.011 \mathrm{~g} / \mathrm{m}^{2}$ for $\mathrm{Na} ; 0.216 \pm 0.007 \mathrm{~g} / \mathrm{m}^{2}$ for $\mathrm{Si}$; and $0.264 \pm 0.009 \mathrm{~g} / \mathrm{m}^{2}$ for B. As compared to the EA glass, the average, normalized elemental release for $\mathrm{Al}$ is higher for the AZ-102 Melt 1 glasses, i.e. $0.037 \pm 0.004 \mathrm{~g} / \mathrm{m}^{2}$ and $0.226 \pm 0.009 \mathrm{~g} / \mathrm{m}^{2}$, respectively. Glass chemistry may be the cause of this change in durability as the AZ-102 Melt 1 glass was analyzed at 7.80 weight percent aluminum oxide while the C-104 and EA glasses were analyzed at 2.64 and 3.67 respectively. Aluminum oxide in the glass network in low concentration significantly increases durability, but there can be a point at which this trend reverses and durability decreases because of increased aluminum oxide. This seems to be what is observed in the AZ-102 Melt 1 glass.

The C-104-CCC-1 sample has lower elemental concentration (between 10 to $13 \%$ lower) and a $\mathrm{pH}$ value 0.013 lower than the other two C-104 samples (see Appendix E). This may have been caused by a slight loss of glass sample. This was the first sample to be loaded into the PCT test vessels in the hot cell and there was trouble with static electricity causing the weighed sample to electrostaticly adhere to the weigh container and the vessel. This loss of sample may have caused the slight decrease in release rates measured for this one sample. Following the preparation of this sample, the process was changed to remove any electrostatic charge effects on sample preparation. There also is a similar difference between the AZ-102-CCC-3 sample and the other set of the triplicate tested with it. The sample is slightly higher in its elemental release compared to AZ-102-CCC-sample \#1 and \#2, but the $\mathrm{pH}$ values are all about the same; however, there is no observed reason for why this difference occurred.

One other factor that is generally not taken into account is the difference in density of the glasses when comparing their PCT values. This is generally acceptable because the density differences are in most cases small compared to the differences in durability values. However, in this case the density of C-104 $(2.888 \mathrm{~g} / \mathrm{cc})$ is about $6.8 \%$ greater than that for AZ-102 $(2.703 \mathrm{~g} / \mathrm{cc})$. For a given weight of glass, this translates directly into a $6.8 \%$ difference in surface area between the glass samples with the C-104 sample having $6.8 \%$ less area than AZ-102. Table 4.21 also gives the density corrected values for C-104. The correction affects the second decimal place, but this is not enough to change any of the conclusions drawn from these data. 
Table 4.20. Comparison of $90^{\circ} \mathrm{C}$ PCT Results from the Environmental Assessment (EA) Glass Round Robin (Jantzen, et al. 1993) with the Equivalent Values for the $90^{\circ} \mathrm{C}$ PCT in this Report

\begin{tabular}{|c|c|c|}
\hline & \multicolumn{2}{|c|}{ EA Glass at $90^{\circ} \mathrm{C}$} \\
\hline Element & $\begin{array}{c}\text { Reported }^{(\mathrm{a})} \\
\text { (mg/L) }\end{array}$ & Measured $(\mathrm{mg} / \mathrm{L})$ \\
\hline [B] & $587 \pm 43$ & $606-325$ \\
\hline$[\mathrm{Na}]$ & $1662 \pm 112$ & $1580-888$ \\
\hline [Li] & $190 \pm 14.5$ & $182-112$ \\
\hline$[\mathrm{Si}]$ & $893 \pm 86$ & $940-647$ \\
\hline Final pH & $11.85 \pm 0.1$ & $11.77-11.33$ \\
\hline
\end{tabular}

Table 4.21. Average 7-Day $90^{\circ} \mathrm{C}$ PCT Normalized Mass Loss Data of Radioactive IHLW Glasses

\begin{tabular}{|c|c|c|c|c|c|c|}
\hline \multicolumn{7}{|c|}{ Average 7-DAY 90 ${ }^{\circ} \mathrm{C}$ PCT Normalized Mass Loss $\left(\mathrm{g} / \mathrm{m}^{2}\right)$} \\
\hline \multirow[b]{2}{*}{ Glass } & \multicolumn{6}{|c|}{ Element } \\
\hline & B & $\mathbf{N a}$ & $\mathbf{L i}$ & Al & Si & pH \\
\hline C-104 & $0.436 \pm 0.035$ & $0.366 \pm 0.020$ & $0.453 \pm 0.024$ & $0.055 \pm 0.005$ & $0.231 \pm 0.014$ & $8.98 \pm 0.076$ \\
\hline C-104 (D corr.) & 0.466 & 0.391 & 0.484 & 0.059 & 0.247 & - \\
\hline AZ-102 Melt 1 & $0.264 \pm 0.008$ & $0.396 \pm 0.011$ & $0.375 \pm 0.008$ & $0.226 \pm 0.009$ & $0.216 \pm 0.007$ & $9.72 \pm 0.020$ \\
\hline EA Glass & $6.90 \pm 2.05$ & $5.05 \pm 1.40$ & $3.75 \pm 0.88$ & $0.037 \pm 0.004$ & $1.77 \pm 0.326$ & $11.58 \pm 0.23$ \\
\hline \multicolumn{7}{|c|}{$\begin{array}{l}\text { Note: ORP Phase B-1 contract requirement from Specification 1, WAPS specification 1.3.1. titled Acceptance } \\
\text { Criterion reads: "For acceptance, the mean concentrations of lithium, sodium and boron in the leachate ... One } \\
\text { acceptable method of demonstrating that the acceptance criterion is met, would be to ensure that the mean PCT } \\
\text { results for each waste type are at least two standard deviations below the mean PCT results of the EA glass. The } \\
\text { " } \pm " \text { values are one standard deviation. }\end{array}$} \\
\hline
\end{tabular}

Table 4.22 compares the average, normalized elemental release rates found for glasses $\mathrm{C}-104$ and AZ-102 Melt 1, at $90^{\circ} \mathrm{C}$ compared to $90^{\circ} \mathrm{C}$ PCT data for the EA glass, HLW98-51R (simulant C-104 glass) glass, and the HLW98-61 (simulant AZ-102 glass) glass provided by VSL. The fact that the VSL glasses were quenched (not heat treated), and the PCT results are similar to those from the glasses made using real waste and heat treated suggests that CCC cooling of the glass will have negligible affect on the chemical durability of these glasses. For PCT-A, the S/V was assumed to be $2000 \mathrm{~m}^{-1}$ based on assumptions on the size and shape distribution of the ground glass and verified through considerable surface area measurements (ASTM C1285-97). Normalized releases of Na, Si, and B were calculated using Equation 1 and are based on a seven-day test period. As is readily observed based upon results from Tables 4.20 and 4.22, all of the glasses easily meet the ORP Phase B-1contract requirement from WAPS specification 1.3.1 titled "Acceptance Criterion" which reads: "The consistency of the waste form shall be demonstrated using the Product Consistency Test (PCT) [3]. For acceptance, the mean concentrations of lithium, sodium and boron in the leachate, after normalizing for the concentrations in the glass, shall each be less than those of the benchmark glass described in the Environmental Assessment for selection of the DWPF waste form [4]. The measured or projected mean PCT results for lithium, sodium and boron shall be provided in the Production Records. The Producer shall define the statistical significance, of the reported data in the WQR. One acceptable method of demonstrating that the acceptance criterion is met, would be to ensure that the mean PCT results for each waste type are at least two standard deviations below the mean PCT results of the EA glass." as the average, normalized 
elemental release rates are an order of magnitude more durable for $\mathrm{Si}, \mathrm{Na}$, and $\mathrm{B}$, and just slightly less than an order of magnitude below for Li when compared to the reported results of the benchmark EA glass (Jantzen et al., 1993).

Table 4.22. Comparison of Average 7-Day $90^{\circ} \mathrm{C}$ PCT Normalized Mass Loss Data Between VSL Non-radioactive Simulant Glasses and Actual Radioactive HLW Glass Counterparts from this Study. Environmental Assessment (EA) Data is Provided for Comparison Purposes

\begin{tabular}{|c|c|c|c|}
\hline \multicolumn{4}{|c|}{ Average 7-DAY $90^{\circ} \mathrm{C}$ PCT Normalized Mass Loss $\left(\mathrm{g} / \mathrm{m}^{2}\right)$} \\
\hline \multirow[b]{2}{*}{ Glass } & \multicolumn{3}{|c|}{ Element } \\
\hline & $\mathbf{B}$ & $\mathbf{N a}$ & Si \\
\hline EA Glass ${ }^{(a)}$ & 7.577 & 5.340 & 1.679 \\
\hline $\begin{array}{l}\text { HLW98-51R }{ }^{(\mathrm{a}, \mathrm{b})} \text { (simulant C-104 glass) } \\
\text { Leachate } \mathrm{pH}=10.55 \text { (Avg. of } 3 \text { samples) }\end{array}$ & 1.3573 & 0.9156 & 0.4074 \\
\hline $\mathrm{C}-104$ (leachate $\mathrm{pH}$ range $=8.89-9.03)$ & 0.436 & 0.366 & 0.231 \\
\hline $\begin{array}{l}\text { HLW98-61 }{ }^{(\mathrm{a}, \mathrm{c})} \text { (simulant AZ-102 glass) } \\
\text { Leachate } \mathrm{pH}=11.01 \text { (Avg. of } 3 \text { samples) }\end{array}$ & 0.2324 & 0.3472 & 0.2751 \\
\hline AZ-102 (leachate $\mathrm{pH}$ range $=9.70-9.74)$ & 0.264 & 0.396 & 0.216 \\
\hline \multicolumn{4}{|c|}{$\begin{array}{l}\text { Note: ORP Phase B-1 contract requirement from Specification 1, WAPS specification 1.3.1. titled Acceptance } \\
\text { Criterion reads: "For acceptance, the mean concentrations of lithium, sodium and boron in the leachate ... One } \\
\text { acceptable method of demonstrating that the acceptance criterion is met, would be to ensure that the mean } \\
\text { PCT results for each waste type are at least two standard deviations below the mean PCT results of the } \\
\text { EA glass. } \\
\text { (a) PCT data provided by VSL; data is from testing completed from March through June } 2000 \text {, all triplicate } \\
\text { sampling in accordance with ASTM C1285-97. PCT was measured using quenched glass samples. } \\
\text { (b) The density of HLW98-51R, provided by VSL, was measured at } 20^{\circ} \mathrm{C} \text { using ASTM D854-83 and is } 2.888 \\
\mathrm{~g} / \mathrm{cm}^{3} \\
\text { (c) The density of HLW98-61, provided by VSL, was measured at } 20^{\circ} \mathrm{C} \text { using ASTM D854-83 and is } 2.703 \mathrm{~g} / \mathrm{cm}^{3}\end{array}$} \\
\hline
\end{tabular}




\subsection{Conclusions}

The primary objective for vitrifying the HLW samples was to demonstrate The RPP-WTP projects ability to satisfy the IHLW product ORP Phase B-1 contract requirements concerning, chemical and radionuclide reporting, waste loading, identification and quantification of crystalline and noncrystalline phases, and waste form leachability. Two pretreated tank sludge samples, high-level wastes (241-C-104 and 241-AZ-102) along with a HLW process simulant (termed the HLW Process Blank) were prepared as melter feeds for vitrification. Due to scheduling constraints and small initial sample size of the pretreated tank 241-AZ-102 sludge, this sample was divided into two samples that were vitrified separately (i.e. AZ-102, Melt 1 and AZ-102, Melt 2). The analyzed compositions of the pretreated C-104 and AZ-102 sludge wastes were used by Catholic University of America's (CUA) Vitreous State Laboratory (VSL) to calculate the target glass compositions. The two tank sludge samples, i.e. C-104 and AZ-102, were processed through pretreatment chemical washing and leaching processes, and the pretreated sludges were converted to high-level waste glass after flowsheet quantities of secondary wastes, i.e. Sr/TRU precipitate and Cs and Tc ion exchange eluants, generated from LAW supernatant pretreatment unit operations and glass former minerals were added.

One of the objectives for the glass product waste loading for the HLW glasses was to meet the requirements delineated in the RPP-WTP Phase B-1 contract, specification 1.2.2.1.6, titled Product Loading, which states: "Loading of non-volatile components in Envelope D, and, if directed by DOE, entrained solids after washing in accordance with Specification 12, Number of HLW Canister Per Batch of Waste Envelope D, shall be achieved, such that, the concentration of at least one of the waste components or waste component combinations in Table TS-1.1 Minimum Component Limits in HLW Glass exceeds its minimum weight percent in HLW glass as identified in Table TS-1.1 ..." The total of all waste oxides (exclusive of Si) not identified in Table TS-1.1 was calculated for the C-104 glass, taking into account the glass former minerals added, using the "Average Normalized" weight percent oxide values. The total waste percent total oxide in the $\mathrm{C}-104$ glass, per the above conditions, comes to $10.02 \%$, which is greater than the required $8.0 \%$. The total of $\mathrm{Al}_{2} \mathrm{O}_{3}+\mathrm{Fe}_{2} \mathrm{O}_{3}+\mathrm{ZrO}_{2}$ for AZ-102 Melt 1 is $21.96 \%$ and for AZ-102 Melt 2 is $21.61 \%$ which is greater than the required $21.0 \%$. Therefore, the C-104, AZ-102 Melt 1, and AZ-102 Melt 2 glasses easily meet the ORP contract specifications for waste product loading.

The waste loading was calculated from the dilution factor (decrease in concentration) of elements contained in either the waste or the glass forming additives. The results indicate that the waste fraction of each glass is near their target, i.e. 33.1\% for C-104 (measured 33.59\% based on waste dilution and $31.05 \%$ based on additive dilution), 33.39\% for AZ-102 Melt 1 (measured 33.14\% based on waste dilution and $34.16 \%$ based on additive dilution), and 33.97\% for AZ-102 Melt 2 (measured 33.98\% based on waste dilution and $34.22 \%$ based on additive dilution). The measured glass to target composition percent difference comparison of the oxides is small and the calculated waste loading values are very close to or exceed the target. Both support the conclusion that the actual waste loading in each glass met or exceeded the target waste loading.

To demonstrate that the IHLW glass product, radionuclide compositional contract criteria were met, it was assumed that each HLW glass, i.e. C-104, AZ-102 Melt 1, and AZ-102 Melt 2, are separate "waste types" and as such would fill multiple Hanford HLW canisters and that the HLW canister can be modeled as a right circular cylinder of $4.5 \mathrm{~m}$ height and $0.61 \mathrm{~m}$ diameter with a $100 \%$ glass fill of approximately $1.27 \mathrm{~m}^{3}$. The primary success objectives accomplished with this work are: 1) "the inventory of radionuclides (in Curies) that have half-lives longer than 10 years and that are, or will be, present in concentrations greater than 0.05 percent of the total radioactive inventory for each waste type, indexed to the years 2015 and 3115 " are reported; 2) the total and fissile uranium and plutonium (U-233, 
U-235, Pu-239, and Pu-241) content of each canister of waste glass were calculated and are: 1161.5 grams for C-104, 642.1 grams for AZ-102 Melt 1, and 619.4 grams for AZ-102 Melt 2; 3) the concentration of plutonium in grams per cubic meter of each waste glass are: $124.7 \mathrm{~g} / \mathrm{m}^{3}$ for C-104, 122.4 $\mathrm{g} / \mathrm{m}^{3}$ for AZ-102 Melt 1, and $122.1 \mathrm{~g} / \mathrm{m}^{3}$ for AZ-102 Melt 2, none of which exceed the contract plutonium loading of 2500 grams per cubic meter; and lastly 4) The ratio by weight of the total element of the following isotopes: U-233, U-234, U-235, U-236, U-238, Pu-238, Pu-239, Pu-240, and Pu-241, are reported. The above IHLW glass product, radionuclide compositional data demonstrates that all three glasses pass the ORP Phase B-1 contract criteria.

Identification and quantification of crystalline and non-crystalline phases were completed by using x-ray diffraction (XRD), optical microscopy, and scanning electron microscopy (SEM) on samples given a slow cool down heat treatment which simulated the calculated cooling profile for glass at the centerline of a Hanford HLW canister during filling. No crystals were observed in the C-104 sample by XRD, optical examination, and SEM analysis. XRD analysis combined with optical microscopy and SEM EDS results of the CCC heat-treated AZ-102 Melt 1 glass sample indicated the presence of a crystalline phase at approximately 1 to 2 volume percent. An XRD search match analysis of the major peaks found in the XRD pattern of the CCC heat-treated AZ-102 Melt 1 glass sample indicated trevorite $\left(\mathrm{NiFe}_{2} \mathrm{O}_{4}\right)$ and chromite $\left(\mathrm{FeCr}_{2} \mathrm{O}_{4}\right)$ as the most likely spinel crystals. The small amount of crystalline material in the CCC heat-treated AZ-102 Melt 1 glass sample does not significantly alter the leaching resistance of the glass as indicated by the PCT test results. SEM examination of both the C-104 and AZ-102 Melt 1 CCC heat-treated glasses at magnifications up to 5000× showed homogenous glass with no evidence of any phase separation.

The Product Consistency Test (PCT) was employed to gauge the IHLW glass chemical durability. The PCT was run at $90^{\circ} \mathrm{C}$, using HLW glass samples given a slow cool down heat treatment that simulates the cooling profile for glass at the center line of a canister being filled with waste glass, to determine the normalized release of lithium, sodium, and boron. The environmental assessment (EA) benchmark standard glass was included in these tests to provide a reliable baseline of results by which to judge the quality of the PCT results for the C-104 and AZ-102 Melt 1 glasses. The normalized lithium, sodium and boron $90^{\circ} \mathrm{C}$ PCT release rates for the C-104, AZ-102 Melt 1, and EA glasses are: 1) (for lithium) $0.5 \mathrm{~g} / \mathrm{m}^{2}, 0.4 \mathrm{~g} / \mathrm{m}^{2}$, and $3.75 \mathrm{~g} / \mathrm{m}^{2}$; 2) (for sodium) $0.4 \mathrm{~g} / \mathrm{m}^{2}, 0.4 \mathrm{~g} / \mathrm{m}^{2}$, and $5.1 \mathrm{~g} / \mathrm{m}^{2}$; and 3 ) (for boron) $0.4 \mathrm{~g} / \mathrm{m}^{2}, 0.3 \mathrm{~g} / \mathrm{m}^{2}$, and $6.9 \mathrm{~g} / \mathrm{m}^{2}$; respectively. More importantly, as the average, normalized elemental release rates are an order of magnitude more durable for $\mathrm{Na}$, and $\mathrm{B}$, and just slightly less than an order of magnitude below for $\mathrm{Li}$ when compared to the reported results of the benchmark EA glass (Jantzen et al., 1993), the ORP Phase B-1 contract criteria were easily met.

Finally, the IHLW product testing results from the C-104 and AZ-102 glasses show that in all cases they meet or exceed ORP Phase B-1 contract specifications for waste loading, chemical composition documentation, radionuclide concentration limitations, and waste form testing (i.e. chemical durability). 


\subsection{Acknowledgements}

Our thanks go out to a number of individuals. This task would not have been accomplished without the many dedicated staff that supported this effort. The work was a challenge and many first-time accomplishments were made. Obstacles to completing the task were worked out by people going above and beyond the call of duty to make things happen. We wish to acknowledge this effort and thank those individuals.

The staff at the Shielded Facility Operations (SFO) are commended on their efforts and willingness to "go the extra mile." Otherwise, there would have been many long delays. Ralph Lettau's efforts in the hot cell operations were key to the good job completed. His coworkers, Mac Zumhoff, Don Rinehart, F.Vaughn Hoopes, and Michael Mann, were instrumental in their support of the work as well. Elena Hernandez, SFO Administrative Assistant, provided important behind the scenes help.

Tim Reining and Paul MacFarlan showed fine work and expertise in making the thin sections of HLW glass. Steve Marschman provided the facilities for grinding and polishing of the glass.

Danny Sanders, Marilyn Steele, May-Lin Thomas, and Jerry Wagner of the Chemical Measurements Center made many efforts to support our inorganic analysis needs. Radiochemical analysis of the glass products by Larry Greenwood, Richard Ratner, Sandy Fiskum, Truc Trang-Le, Katharine Carson, Jackie Deschane, Chuck Soderquist, and Kathie Thomas are greatly appreciated.

The craftsmen who supported this task deserve thanks. There were many who helped, and several made key contributions in this task. Jim Sportelli 'made things happen' and Michael Krouse went out of his way to help at critical times by producing machined parts that required a lot of thought and interaction with project staff. Dennis F. Smith and Larry D. Lang provided significant help with timely repair of broken manipulators. Tom Powell, Dave Hebert and Michael Blakley spent many patient hours preparing the high temperature furnace for remote operating and introduction into the hot cell. Tony (Anthony) Martin, Stu Murphy, and Monte Fleming were instrumental in the final electrical modifications and in-cell preparation of the furnace.

Evan Jenson provided timely X-ray diffraction analysis of the radioactive glass samples. Bruce Arey did an excellent job of analyzing the radioactive glass samples for crystalline phases and glass homogeneity by scanning electron microscopy.

The Thermal Processing Group supported our efforts by covering for staff in many instances while they were working on the project. Loni Peurrung provided important help with safety issues. Ginger Rhoades' and Teresa Schott's efforts helped keep the project moving when it was most needed. And thanks to Glass Development Laboratory staff, especially John Vienna, Jarrod Crum, and Donald E. Smith, who had a lot more work to do because of staff pulled away for this project. 


\subsection{References}

American Society for Testing and Materials, ASTM C1285-97 "Standard Test Methods for Determining Chemical Durability of Nuclear, Hazardous, and Mixed Waste Glasses: The Product Consistency Test (PCT)", West Conshohoken, Pennsylvania.

Arm, Stuart T, BNFL, E-mail to Gary L. Smith, Battelle, dated December 20, 1999, titled "Preliminary IHLW Cooling Curve", CH2MHill Hanford Group (CHG) reference number: 015943C, October 2000.

Blanchard Jr., D. L., D. E. Kurath, and J. R. Bontha. 1999. Small Column Testing of Superlig 639 for Removal of ${ }^{99}$ Tc from Hanford Tank Waste Envelope A (Tank 241-AW-101). BNFL-RPT-016. PNWD-3004. Pacific Northwest National Laboratory, Richland, Washington.

Blanchard Jr., D. L., D. E. Kurath, and B. M. Rapko. 2000. Small Column Testing of Superlig 639 for Removing ${ }^{99}$ Tc from Hanford Tank Waste Envelope C (Tank 241-AN-107). BNFL-RPT-022.

PNWD-3028. Pacific Northwest National Laboratory, Richland, Washington.

Bredt, P. R., L. K. Jagoda, and D. E. Rinehart. 2000. Rheological Studies on Pretreated Feed and Melter Feed from C-104 and AZ-102. WTP-RPT-004, Rev. 0.PNNL-13359. Pacific Northwest National Laboratory, Richland, Washington.

Brooks, K. P., P. R. Bredt, G. R. Golcar, S. A. Hartley, L. K. Jagoda, K. G. Rappe, and M. W. Urie. 2000a. Characterization, Washing, Leaching, and Filtration of C-104 Sludge. BNFL-RPT-030, Rev. 0. PNWD-3024. Pacific Northwest National Laboratory, Richland, Washington.

Brooks, K. P., P. R. Bredt, S. K. Cooley, G. R. Golcar, L. K. Jagoda, K. G. Rappe, and M. W. Urie. 2000b. Characterization, Washing, Leaching, and Filtration of AZ-102 Sludge. BNFL-RPT-038, Rev. 0. PNWD-3045. Pacific Northwest National Laboratory, Richland, Washington.

Browne, Edgardo and Richard B. Firestone. 1986. Table of Radioactive Isotopes. Virginia S. Shirley, editor, published by John Wiley \& Sons, Inc., New York, New York.

Crawford, Charles L., Daro M. Ferrara, Bao C. Ha, and Ned E. Bibler. 1998. Production of a High-Level Waste Glass from Hanford Waste Samples, in Proceedings of the International Conference on "Decommissioning and Decontamination and on Nuclear and Hazardous Waste Management," pp. 581-588. Spectrum '98 Vol. 1, American Nuclear Society, Inc., La Grange Park, Illinois.

Deming, W. Edwards. "Statistical Adjustment of Data". Dover Publications, Inc. 1964.

Evans, J. C., J. A. Campbell, F. V. Hoopes, E. W. Hoppe, G. S. Klinger, G. M. Mong, A. K. Sharma, M. J. Steele, R. T. Steele, M. W. Urie. 2000. Organic Analysis of C-104 Tank Waste. WTP-RPT-008. PNNL-(TBD). Pacific Northwest National Laboratory, Richland, Washington.

Ferrara, D. M., C. L. Crawford, B. C. Ha, N. E. Bibler and A. S. Choi. 1998. Vitrification of Four Radioactive Hanford Waste Samples (U). SRTC-BNFL-023, Rev. 2. Savannah River Technology Center, Aiken, South Carolina. 
Fiskum, S. K., C. J. Barinaga, J. P. Bramson, K. J. Carson, J. R. DesChane, O. T. Farmer, L. R. Greenwood, F. V. Hoopes, R. T. Ratner, D. R. Sanders, M. J. Steele, R. T. Steele, C. Z. Soderquist, R. W. Swoboda, K. K. Thomas, T. L. Trang-Le, M. W. Urie, and J. J. Wagner. 2000. Inorganic and Radiochemical Analysis of 241-C-104 Tank Waste. WTP-RPT-007. PNNL-13364. Pacific Northwest National Laboratory, Richland, Washington.

Hallen, Richard T., Paul R. Bredt, Kriston P. Brooks, and Lynette K. Jagoda. 2000. Combined Entrained Solids and Sr/TRU Removal from AN-107 Diluted Feed. BNFL-RPT-027. PNWD-3035. Pacific Northwest National Laboratory, Richland, Washington.

Hahn, Gerald J. and William Q. Meeker. Statistical Intervals: A Guide for Practitioners. John Wiley \& Sons, 1991.

HWMA. Hazardous Waste Management Act.

Jantzen, C. M., N. E. Bibler, D. C. Beam, C. L. Crawford, and M. A. Pickett. 1993. Characterization of the Defense Waste Processing Facility (DWPF) Environmental Assessment (EA) Glass Standard Reference Material. WSRC-TR-92-346, Rev. 1. Westinghouse Savannah River Company, Aiken, South Carolina.

Kurath, D. E., D. L. Blanchard, and J. R. Bontha. 2000a. Small Column Ion Exchange Testing of Superlig 644 for Removal of ${ }^{137}$ Cs from Hanford Tank Waste Envelope C (Tank 241-AW-101). BNFL-RPT-014. PNWD-3001. Pacific Northwest National Laboratory, Richland, Washington.

Kurath, D. E., D. L. Blanchard, and JR Bontha. 2000b. Small Column Ion Exchange Testing of Superlig 644 for Removal of ${ }^{137}$ Cs from Hanford Tank Waste Envelope C (Tank 241-AN-107). BNFL-RPT-024. PNWD-3039. Pacific Northwest National Laboratory, Richland, Washington.

Lumetta, G. J., D. J. Bates, J. P. Bramson, L. P. Darnell, O. T. Farmer III, S. K. Fiskum, L. R. Greenwood, F. V. Hoopes, C. Z. Soderquist, M. J. Steele, R. T. Steele, M. W. Urie, and J. J. Wagner. 2000. C-104 High-Level Waste Solids: Washing/Leaching and Solubility Versus Temperature Studies. BNFL-RPT-021. PNWD-3027. Pacific Northwest National Laboratory, Richland, Washington.

PNNL Operating Procedure, PNL-ALO-103, Rev. 1, 1990. "Water Leach of Sludges and Soils", Pacific Northwest National Laboratory, Richland, Washington.

PNNL Operating Procedure, PNL-ALO-114, Rev 1, 1995. "Solubilization of Metals from Solids Using Na2O2 Fusion", Pacific Northwest National Laboratory, Richland, Washington.

PNNL Operating Procedure, PNL-ALO-115, Rev 1, 1995. "Solubilzatioin of Metals from Solids Using KOH-KNO3 Fusion", Pacific Northwest National Laboratory, Richland, Washington.

PNNL Operating Procedure, PNL-ALO-211, Rev. 0, 1991. "Determination of Elements by Inductively Coupled Argon Plasma Atomic Emission Spectrometry”, Pacific Northwest National Laboratory, Richland, Washington.

PNNL Operating Procedure, PNL-ALO-212, Rev. 1, 1992. "Determination of Inorganic Anions by Ion Chromatography”, Pacific Northwest National Laboratory, Richland, Washington. 
PNNL Operating Procedure, PNL-ALO-280, Rev. 1, 1993. "Inductively Coupled Argon Plasma Atomic Emission Spectrometry (ICP/MS) Analysis”, Pacific Northwest National Laboratory, Richland, Washington.

PNNL Operating Procedure, PNL-ALO-281, Rev. 1, 1993. “(ICP/MS) Determination of 99Tc”, Pacific Northwest National Laboratory, Richland, Washington.

PNNL Operating Procedure, PNL-ALO-282, Rev. 1, 1996. "Determination of Uranium Concentration/Isotopic Composition Using ICP/MS", Pacific Northwest National Laboratory, Richland, Washington.

PNNL Operating Procedure, PNL-ALO-381, Rev. 1, 1995. "Direct Determination of TC, TOC, and TIC in Radioactive Sludges and Liquids by Hot Persulfate Method", Pacific Northwest National Laboratory, Richland, Washington.

PNNL Operating Procedure, RPG-CMC-408, Rev 0, 1999. "Total Alpha and Beta Analysis", Pacific Northwest National Laboratory, Richland, Washington.

PNNL Operating Procedure, PNL-ALO-417, Rev 2. 1996. "Separation of U, Am/Cm, and Pu and Actinide Screen by Extraction Chromatography", Pacific Northwest National Laboratory, Richland, Washington.

PNNL Operating Procedure, PNL-ALO-422, Rev 0, 1992. "Solution Analysis: Alpha Spectrometry", Pacific Northwest National Laboratory, Richland, Washington.

PNNL Operating Procedure, PNL-ALO-432, Rev. 0, 1992. "Separation of Technetium by Cation Exchange and Solution Extraction Prior to Measurement by Beta Counting", Pacific Northwest National Laboratory, Richland, Washington.

PNNL Operating Procedure, PNL-ALO-450, Rev. 1, 1993. "Gamma Energy Analysis (GEA) and LowEnergy Photon Spectrometry (LEPS)”, Pacific Northwest National Laboratory, Richland, Washington.

PNNL Operating Procedure, PNL-ALO-476, Rev. 1, 1995. "Strontium Determination Using SR-SPEC", Pacific Northwest National Laboratory, Richland, Washington.

PNNL Operating Procedure, PNL-ALO-496, Rev 1, 1994. "Precipitation Plating of Actinides for HighResolution Alpha Spectrometry", Pacific Northwest National Laboratory, Richland, Washington.

QARD. DOE/RW-0333P. Rev. 8. November 13, 1997. Quality Assurance Requirements and Description for the Civilian Radioactive Waste management Program (QARD). U.S. Department of Energy, Office of Civilian Radioactive Waste Management. Washington, D.C.

RCRA. Resource Conservation and Recovery Act.

Smith, G. L. 1993. Characterization of Analytical Reference Glass-1 (ARG-1). PNL-8992. Pacific Northwest National Laboratory, Richland, Washington.

Smith, G. L., D.L. Eggett, and H.D. Smith, "Waste Glass Analytical Bias Correction Using a Reference Standard Glass," in Scientific Basis for Nuclear Waste Management XX, Vol. 465, 173-180, ed. by W.J. Gray and I. Triay, Materials Research Society, Pittsburgh, Pennsylvania, 1997. 
SW-846, Test Methods for Evaluating Solid Waste, Physical/Chemical Methods, U.S. Environmental Protection Agency, Office of Solid Waste and Emergency Response, Washington, D.C.

WAC 173-303. Dangerous Waste Regulations. Washington Administrative Code, as amended.

WAPS. DOE/EM-0093. Rev. 2. December 1996. Waste Acceptance Product Specifications for Vitrified High Level Waste Forms (WAPS). U.S. Department of Energy, Office of Environmental Management, Washington, D.C.

WASRD. DOE/RW-0351P. Rev. 2. DCN 02. December 1996. Waste Acceptance System Requirements Document (WASRD). U.S. Department of Energy, Office of Civilian Radioactive Waste Management. Washington, D.C. 